\title{
Defect reaction paths of nickel in float zone silicon controlled by non-equilibrium vacancies
}

\author{
Dissertation \\ zur Erlangung des mathematisch-naturwissenschaftlichen Doktorgrades \\ "Doctor rerum naturalium" \\ der Georg-August-Universität Göttingen \\ im Promotionsprogramm PROPHYS \\ der Georg-August University School of Science (GAUSS)
}

vorgelegt von

Philipp Saring

aus Göttingen

Göttingen, 2019 
Betreuungsausschuss

Prof. Michael Seibt, IV. Physikalisches Institut

Georg-August Universität Göttingen

Prof. Hans Hofsäss, II. Physikalisches Institut

Georg-August Universität Göttingen

Mitglieder der Prüfungskommision

Referent: Prof. Michael Seibt, IV. Physikalisches Institut

Korreferent: Prof. Hans Hofsäss, II. Physikalisches Institut

weitere Mitglieder der Prüfungskommision

Prof. Dr. C. Jooß, Institut für Materialphysik

Georg-August Universität Göttingen

Prof. Dr. V. Moshnyaga, I. Physikalisches Institut

Georg-August Universität Göttingen

PD. Dr. M. Wenderoth, IV. Physikalisches Institut

Georg-August Universität Göttingen

Prof. Dr. W. Kollatschny, Institut für Astrophysik

Tag der mündlichen Prüfung: 


\section{Contents}

$\begin{array}{ll}\text { Abstract } & 3\end{array}$

Introduction $\quad \mathbf{5}$

Analysis of nitrogen-vacancy complexes with nickel in-diffusion . 6

Effect of vacancies on nickel disilicide nucleation . . . . . . . . 6

Distribution and electronic properties of individual nickel disilicide nano-structures . . . . . . . . . . . . . 7

1 Nickel in Silicon: A Review 9

1.1 Nickel and vacancies in silicon . . . . . . . . . . . . . . . . 9

1.1.1 High purity float zone silicon . . . . . . . . . . . . . . 9

1.1.2 Vacancy-complexes in silicon . . . . . . . . . . . . . 11

1.1 .3 Nickel in silicon . . . . . . . . . . . . . . . . . . . 12

1.1.4 Nickel in-diffusion for vacancy marker analyses . . . . . . 13

1.2 Nickel disilicide precipitation . . . . . . . . . . . . . . . . . . 17

1.2.1 Early stages of nickel disilicide precipitation . . . . . . 17

1.2 .2 Initial stages . . . . . . . . . . . . . . . . . . 18

1.2.3 Nucleation processes . . . . . . . . . . . . . . . . 18

1.2.4 Determination of the precipitate density . . . . . . . 19

1.3 Electronic properties of $\mathrm{NiSi}_{2}$ precipitates . . . . . . . . . . 20

1.3.1 Previous DLTS analyses of $\mathrm{NiSi}_{2}$ precipitates . . . . . . . 20

1.3.2 Recombination properties of $\mathrm{NiSi}_{2}$ precipitates . . . . . 21

2 Experimental $\quad 25$

2.1 Preparation process . . . . . . . . . . . . . . 25

2.2 Deep Level Transient Spectroscopy (DLTS) . . . . . . . . . . . . 29

2.2.1 Method .................... 29

2.2.2 DLTS measurement details . . . . . . . . . . . . . . 31

2.3 Electron Beam-Induced Current (EBIC) . . . . . . . . . . . . . 32

2.4 Preparation for EBIC analyses of precipitates . . . . . . . . 33

2.5 Focused Ion Beam (FIB) preparation details for cross section EBIC and Transmission Electron Microscopy (TEM) . . . . . . 34 
3 Results and discussion

3.1 Kinetics of grown-in vacancy-related complexes in FZ-silicon and their interaction with nickel atoms . . . . . . . . . . 35

3.1.1 Deep bandgap levels . . . . . . . . . . . . . . . 36

3.1.2 Vacancies tracked with nickel in-diffusion . . . . . . . . . 43

3.1 .3 Annealing stages . . . . . . . . . . . . . . . . . 44

3.1.4 Additional dopants and structural defects: Alternative vacancy reaction paths . . . . . . . . . . . 51

3.1.5 Advantages and drawbacks of the nickel marker in-diffusion technique .................. . 5 54

3.2 Correlation between vacancy concentration and nickel disilicide nucleation . . . . . . . . . . . . . . . . 56

3.2.1 Substitutional nickel increases precipitation . . . . . . 56

3.2.2 Decrease of substitutional nickel concentration under precipitation . . . . . . . . . . . . . . 64

3.2.3 Possible early $\mathrm{NiSi}_{2}$ nucleation stages . . . . . . . . . . 67

3.3 Distribution and electronic properties of individual precipitates . 73

3.3.1 Spatial distribution of precipitates . . . . . . . . . 73

3.3.2 Electron beam induced current profiles of individual large precipitates . . . . . . . . . . . . . 78

3.3.3 Charge collection profiles of individual large precipitates at FIB-prepared cross-sections . . . . . . . . . . . 83

$\begin{array}{lll}4 & \text { Summary } & 91\end{array}$

$\begin{array}{ll}\text { Appendix } & 92\end{array}$

$\begin{array}{lr}\text { A Appendix } & \mathbf{9 3}\end{array}$

A.1 The equation system for nickel diffusion in silicon . . . . . . . . 93

A.1.1 Diffusion reaction kinetics . . . . . . . . . . . . . 93

A.1.2 Rate constants . . . . . . . . . . . . . . . . . 94

A.1.3 Point defect parameters . . . . . . . . . . . . 95

A.2 Determination of the free charge carrier capture kinetics by Pons $[80] \ldots \ldots \ldots \ldots$. . . . . . . . . . . . . . . . . 96

A.2.1 Small deep trap concentrations . . . . . . . . . 96

A.2.2 Large deep trap concentrations . . . . . . . . . . . . . 97

A.3 Sinks for vacancies and nitrogen . . . . . . . . . . . . . . . . 99

A.4 DLTS-graphs of nickel marker in-diffusion in crystalline silicon besides FZ-silicon . . . . . . . . . . . . . . . . . . . . 101

A.5 DLTS graphs from precipitates after different in-diffusion conditions . . . . . . . . . . . . . . . . . . . . 102

A.6 Precipitate density and number of $\mathrm{Ni}$ atoms in precipitate . . . 103

A.7 Energy-dependant EBIC cross section analysis of a $\mathrm{NiSi}_{2}$ precipitate in a ridge structure . . . . . . . . . . . . . . . . . 104

A.8 experimental parameters . . . . . . . . . . . . 106 
Bibliography 


\section{Abstract}

This thesis investigates how defect reaction paths of nickel in float zone silicon can be controlled by non-equilibrium vacancy concentrations. Three different aspects have been studied in detail.

The temperature-dependent annealing kinetics of native vacancy nitrogen complexes in float zone silicon is approached under inert conditions and vacancy detection by nickel marker experiments. The study reveals large concentrations of weakly bound vacancies that irreversibly anneal at medium temperatures.

These experiments open the route to establish non-equilibrium concentrations of substitutional nickel which result in a considerably density of smaller nickel disilicide precipitates in quenching experiments. This provides evidence for an enhanced precipitate nucleation rate catalyzed by substitutional nickel. Possible nucleation stages lowering the nucleation barrier are proposed.

Along these experiments special cooling conditions have been developped which promote the formation of large platelet precipitates with extraordinary aspect ratio of less than $1: 1000$. They form highly coherent interfaces to the silicon host lattice, which originate from a self-aligned growth process. Individual platelets have been excavated from bulk samples by Focused Ion Beam and studied in detail by Electron Beam-Induced Current analysis finding evidence of charge transport properties. 


\section{Introduction}

The ongoing progress in silicon-based technologies is inseparably connected to the efficient control of intrinsic point defects and foreign elements $[1,2]$. Among the latter, nickel plays a prominent role: On the one hand, it is considered as a notorious contaminant that, due to its high diffusivity [3, 4], is accidentally introduced into the material during crystal growth or processing in microelectronics or solar cell production, where it potentially forms detrimental defects. On the other hand, it has gained certain importance for silicide structures, as e.g. in gate-contacts [5], in nanoelectronics.

Several studies analyzed the behaviour of nickel regarding its interactions with silicon, see e.g. $[6,7,8,3,9,10,11,12,13,14]$, inquiring the properties of point defects, precipitation and silicide structures. Common to them all, the behaviour of nickel strongly depends on the processing conditions. Few studies refer to the interaction of nickel in silicon with additional agents such as other transition metals $[2,15,16,17]$ or hydrogen [18]. A rather new issue is the interaction of nickel with additional vacancies. A recent study [19] provides indications that the kinetics of nickel can sensitively be affected by certain asgrown vacancy complexes that result from excess vacancies during the crystal cooling process.

In the present work, effects of non-equilibrium vacancy concentrations on defect reaction paths of nickel and vacancies in float zone (FZ) silicon are analyzed. Different temperature regimes are classified by the following subjects, dividing the analysis in three parts:

- In-grown vacancy complexes in float zone silicon and their interaction with nickel at low temperatures(below $T=700^{\circ} \mathrm{C}$ ).

- Effects of vacancies on nickel disilicide nucleation upon quenching from medium annealing temperatures(temperature range: $750{ }^{\circ} \mathrm{C}-950{ }^{\circ} \mathrm{C}$ ).

- Distribution and electronic properties of individual precipitates, formed upon quenching from high temperatures. 


\section{Analysis of nitrogen-vacancy complexes with nickel in-diffusion}

The properties of highly pure crystalline silicon are sensitively governed by intrinsic point defects, incorporated into the material during crystallization process [20]. Under typical float zone growth conditions for most technological purposes [21], vacancies prevail and supersaturate in the crystal during cooling. Their relaxation by forming detrimental microdefects is commonly suppressed by a controlled supply of nitrogen [22], resulting in smaller and electrically inactive complexes in the as-grown state [23]. Remarkably, it has repeatedly been observed that these vacancy-nitrogen-related complexes in FZ-silicon become electrically active during subsequent wafer-based, thermal processes [24] even in the medium temperature regime [25]. However, the annealing kinetics of these native complexes, possible intermediate states and their electrical properties, are currently unexplored.

An established technique to determine vacancy profiles is to exploit their interaction with suitable marker atoms [26], which are supplied from controlled in-diffusion into the samples. Trapped at a vacancy, they become detectable on substitutional sites in subsequent analytics. Earlier used marker species are characterized by rather high interstitial migration and formation energies, which actually required a rather high thermal budget for the marker indiffusion process, resulting in unwanted side reactions. In recent studies [19], nickel was found to be a sensitive marker for vacancy profiles. Due to rather small formation and migration enthalpies, in-diffusion can be conducted with a rather low thermal budget which provides a high sensitivity to the annealing stages of vacancy-nitrogen complexes in silicon near the as-grown state.

In the first part of the analysis (sec. 3.1), the annealing kinetics of native vacancy-nitrogen-related complexes in FZ-silicon are approached from systematic annealing processes under inert conditions, suitably combined with nickel in-diffusion. Subsequent DLTS analyses enabled to characterize the emerging deep levels and to determine the vacancy concentrations. Different annealing regimes are classified and possible conversion reactions are proposed.

\section{Effect of vacancies on nickel disilicide nucleation}

In many gettering and defect engineering processes, the kinetics of precipitation play a key role [1]. Therefore, an understanding of their formation kinetics is crucial. Recent simulations [27], addressing the development of their size distributions, require concepts for their initial stages. Therefore, a better understanding of the early nucleation process is essential. For the case of copper precipitation, agglomerates with four atoms of three interstitials at one substitutional atom, examined in DLTS analyses [28] and first-principles studies [29] have been proposed as early nucleation stages for copper silicide precipitation. 
Nickel disilicide precipitates form under quenching from high temperatures. Seibt and Schröter [9] established a comprehensive model for the growth mechanism of precipitates under non-equilibrium conditions, considering the incorporation of nickel atoms into a nickel disilicide $\{111\}$ double layer via a bounding partial dislocation under conditions of high supersaturation. Extrapolating the energy of the bounding dislocation towards small stacking fault radii, the nucleation barrier was found to be rather high, compared to the driving chemical potentials from applicable supersaturation levels, which raised the question of the atomic configuration of initial nuclei with sufficiently small energies per atom. Could substitutional nickel be involved?

In the second part of the analysis (sec. 3.2), nickel precipitation is analyzed with and without substitutional nickel controlled by adjusting the pre-existing non-equilibrium vacancies.

\section{Distribution and electronic properties of individual nickel disilicide nano-structures}

Transition metal silicides have advanced to a standard material in siliconbased microelectronic applications. Combining low specific resistivity and low contact resistivity with applicability to both doping types and good process compatibility, they are an essential part of many electronic devices by constituting interconnectors and gate-, source- or drain-contacts [30, 5, 31]. During the continuous miniaturization, specific requirements to thermal stability and low strain have stressed the potential of nickel silicides [32, 33, 34], among which nickel disilicide plays a major role. Pioneering studies on thin films $[35,36,37,38,12,13]$ showed the ability of $\mathrm{NiSi}_{2}$, to grow epitaxially by silicidation of a nickel layer on silicon $<001>$-substrates as well as on silicon $<111>$-substrates, providing perfect single crystallinity and atomically sharp interfaces. Meanwhile, technical advances have enabled the production of various nano-designs such as contacts for nanowire transistors [39, 40] freestanding nanowires [41] or embedded structures with high aspect ratio [42] and excellent transport properties. The ongoing development raises the question, how far this downscaling process can be pushed and where will be the limits.

To this end, the as-quenched $\mathrm{NiSi}_{2}$ precipitates [9] represent the smallest twodimensional $\mathrm{NiSi}_{2}$ structures, whose existence has been reported so far. They are ultrathin self-aligned structures, buried in the silicon matrix with virtually strain-free interfaces. With respect to their electrical properties, the presence of deep levels with bandlike character related to a Coulomb potential has been derived from DLTS-studies, averaging over large ensembles of precipitates $[11$, 43].

In the third part of the analysis (sec. 3.3), individual nickel disilicide precip- 
itates are scrutinized by energy-dependent high-magnification EBIC investigations. Precipitation under sufficiently large supply of interstitial nickel, which we have realized by immediate cooling from high diffusion temperatures at $1100^{\circ} \mathrm{C}$, has enabled the growth of nickel disilicide platelets with diameters of some $10 \mu \mathrm{m}$. Certain regions revealed a thickness of only two monolayers. Analysis in selected spatial arrangements allows the separated investigation of recombination effects at certain parts of the structure respectively and of its vicinity and leads to the approach of charge collection processes at the structures. 


\section{Chapter 1}

\section{Nickel in Silicon: A Review}

\subsection{Nickel and vacancies in silicon}

\subsubsection{High purity float zone silicon}

The growth of silicon crystals with the highest purity - providing the best conditions for controlled processing and defined analytics - is achieved with the float zone method, illustrated in fig. 1.1. A crystalline rod, only mounted at its ends, is refined by moving a liquid zone, created by radio-frequency induction coils, in axial direction through the rod. By this technique, any mechanical contact between the melt and potential contaminant sources of the setup can be avoided. The method makes use of low segregation coefficients of relevant impurities at the crystallization front so that the impurities accumulate in the floating melt region. The crystallization commonly occurs in inert argon ambient, to which, for the purpose of improving crystal mechanical strength, a small fraction of nitrogen of $1.5 \%$ to $2.5 \%$ [21], is added. The low concentration of relevant impurities and dislocations compared to other common growth methods for crystalline silicon is illustrated in table 1.1 .

\begin{tabular}{|c|c|c|c|}
\hline & Float zone & Czochralski & Block cast \\
\hline nitrogen $\left[\mathrm{cm}^{-3}\right]$ & $\approx 10^{15}[45]$ & $<10^{15}[46]$ & $>10^{15}[47]$ \\
oxygen $\left[\mathrm{cm}^{-3}\right]$ & $\leq 5 \cdot 10^{15}$ & $5-9 \cdot 10^{17}[48]$ & $>10^{17}[47]$ \\
dislocations $\left[\mathrm{cm}^{-2}\right]$ & negligible & negligible & $10^{2}-10^{8}[47]$ \\
\hline
\end{tabular}

Table 1.1: Impurity and dislocation concentration after the most prevalent growth methods. The lowest defect concentrations exist in float zone silicon. The defect concentration for float zone silicon refer to specifications by Siltronic. They are expected to hold for most common FZ-material to the actual commercial standard.

Even for the highest purity, crystal growth is inevitably related to intrinsic 


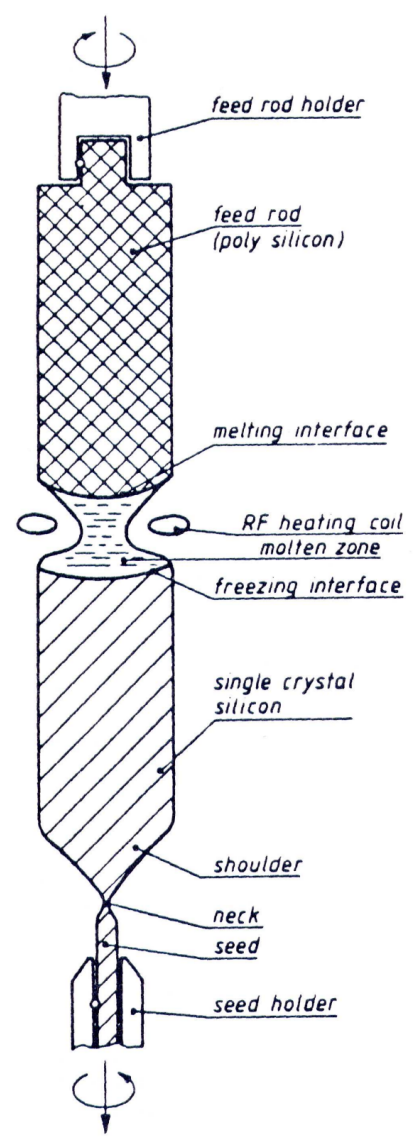

Figure 1.1: Float zone method of silicon growth (after Zulehner [44]).

point defects, i.e. vacancies and self interstitials, created at the crystallization front in thermal equilibrium concentrations and incorporated in the crystal. A comprehensive overview of the detailed reactions of intrinsic point defects during the growth process is given by Falster et al. [20]. They establish the classification in vacancy type growth mode, resulting in vacancy-rich material, and interstitial type growth mode, resulting in interstitial-rich material. The growth modes are distinguished by the crystal growth parameter [49], given by the ratio $\mathrm{V} / \mathrm{G}$ of the growth velocity $\mathrm{V}$ to the temperature gradient $\mathrm{G}$. The pull rates, usually applied in commercial processes, are linked to growth parameters above a critical value $(\mathrm{V} / \mathrm{G})_{\text {crit }}$ and result in vacancy-rich material. 


\subsubsection{Vacancy-complexes in silicon}

A vacancy designates a missing atom in the crystal. At cryogenic temperatures, isolated vacancies in silicon have been comprehensively studied by Watkins and coworkers, providing knowledge about their charge states, Jahn-Teller relaxations, corresponding time constants and activation energies for reorientation and diffusion $[50,51,52,53])$. At room temperature, vacancies practically are not stable on isolated sites. Due to their high mobility, excess vacancies inevitably interact with the specimen surface, agglomerate or form complexes with impurities.

General statements concerning the complexing reactions of vacancies are hampered by the fact that there are various suggestions for the binding energies for vacancy complexes, see Pichler [54], pp. 129-131. Even the parameters for the equilibrium concentration of isolated vacancies, reviewed in Pichler [54], pp.107-113, deviate among different experiments by some orders of magnitude.

Regardless of these controversies there exist unique results for the concentration of vacancies in relation to the concentration of self-interstitials from crystal growth analyses. At the melting temperature, the equilibrium concentration of vacancies, incorporated at the crystallization front exceeds that of self-interstitials by about $2.3 \times 10^{14} \mathrm{~cm}^{-3}[20]$. In the typically applied vacancy-rich growth mode, kinetics during subsequent cooling is mainly dominated by Frenkel-recombination of vacancies with the available self-interstitials whereas excess vacancies remain in the crystal. They show a strong tendency to form detrimental agglomerates such as voids (common synonyms are Ddefects, COPs or GOI [20]), during the further cooling.

For an effective vacancy defect engineering, foreign agents have gained major importance: In Czochralski $(\mathrm{Cz})$ silicon, inherent oxygen concentrations around $10^{17} \mathrm{~cm}^{-3}$, established in equilibrium with the crucible, promote the formation of the uncritical $\mathrm{VO}_{2}$-complex [20], which is electrically inactive and sufficiently stable. In float zone (FZ) silicon, containing per se much smaller oxygen concentrations, the efficient suppression of voids is one key benefit (see Pichler [54], p. 378) of the additional doping with nitrogen. It is well-recognized, that the effect is based on the consumption of vacancies via nitrogen vacancy complexes. The underlying point defect kinetics are, however, ambiguous, which might be due to the great variety of possible defects. Different stoichiometries for $\mathrm{V}$ and $\mathrm{N}$ in such complexes have been proposed $[55,56,45,57]$. A comprehensive picture of the principal complexes is still missing. Beyond the knowledge that these structures are electrically inactive in the as-grown state, their annealing kinetics, particularly their initial conversion reactions with respect to the recently observed degradation effects [25] are largely unexplored yet. 


\subsubsection{Nickel in silicon}

Among the 3d transition metals, nickel stands out for high interstitial solubility and diffusivity. Whereas interstitial nickel is assumed to be neutral, substitutional nickel is associated with a certain electrical activity with deep levels given below.

NAA measurements after nickel in-diffusion experiments in the temperature range between $500^{\circ} \mathrm{C}$ and $1000^{\circ} \mathrm{C}$, presented by E.R. Weber [3], revealed nickel concentrations according to

$$
[N i]_{i}^{e q}=5 \cdot 10^{22} \mathrm{~cm}^{-3} \times \exp \left(3.2-\frac{1.68 \mathrm{eV}}{k_{B} T}\right)
$$

attributed to the interstitial nickel solubility $N i_{s}$ in thermal equilibrium with nickel disilicide boundary phase formed at the silicon-metal interface.

In the range between $800{ }^{\circ} \mathrm{C}$ and $1300{ }^{\circ} \mathrm{C}$ the diffusivity, reviewed in Weber $[3]$, is given by [7]

$$
D(T)=2 \cdot 10^{-3} \frac{\mathrm{cm}^{2}}{\mathrm{~s}} \times \exp \left(-\frac{0.47 \mathrm{eV}}{k_{B} T}\right) .
$$

Based on explicit observations of rapid nickel diffusion in industrial applications, recent measurements were performed [4] in the temperature range between $650{ }^{\circ} \mathrm{C}$ and $900^{\circ} \mathrm{C}$ which revealed the diffusivity given by

$$
D(T)=1.69( \pm 0.74) \cdot 10^{-4} \frac{\mathrm{cm}^{2}}{\mathrm{~s}} \times \exp \left(-\frac{0.15( \pm 0.04) \mathrm{eV}}{k_{B} T}\right)
$$

The migration enthalpy determined in that study is close to theoretical predictions [58] from first-principles simulations with the nudged-elastic-band approach $(0.21 \mathrm{eV})$. The extrapolation of the diffusivity with such small migration enthalpy leads to remarkably large values at room temperature.

In thermal equilibrium, electrically active nickel has been found in Hall measurements with concentrations [6] of

$$
[N i]_{s}^{e q}=10^{26} \mathrm{~cm}^{-3} \times \exp \left(\frac{-3.1 \mathrm{eV}}{k_{B} T}\right)
$$


The detected species has been related to substitutional nickel, which is broadly accepted nowadays, corresponding to deep levels at $E_{c}-0.06 \mathrm{eV}, E_{c}-0.43 \mathrm{eV}$ (including the thermal activation of the capture cross section), and $E_{v}+$ $0.15 \mathrm{eV}$, reviewed by Schröter and Seibt [59]. The corresponding DLTS-lines are shown in fig. 1.2 .

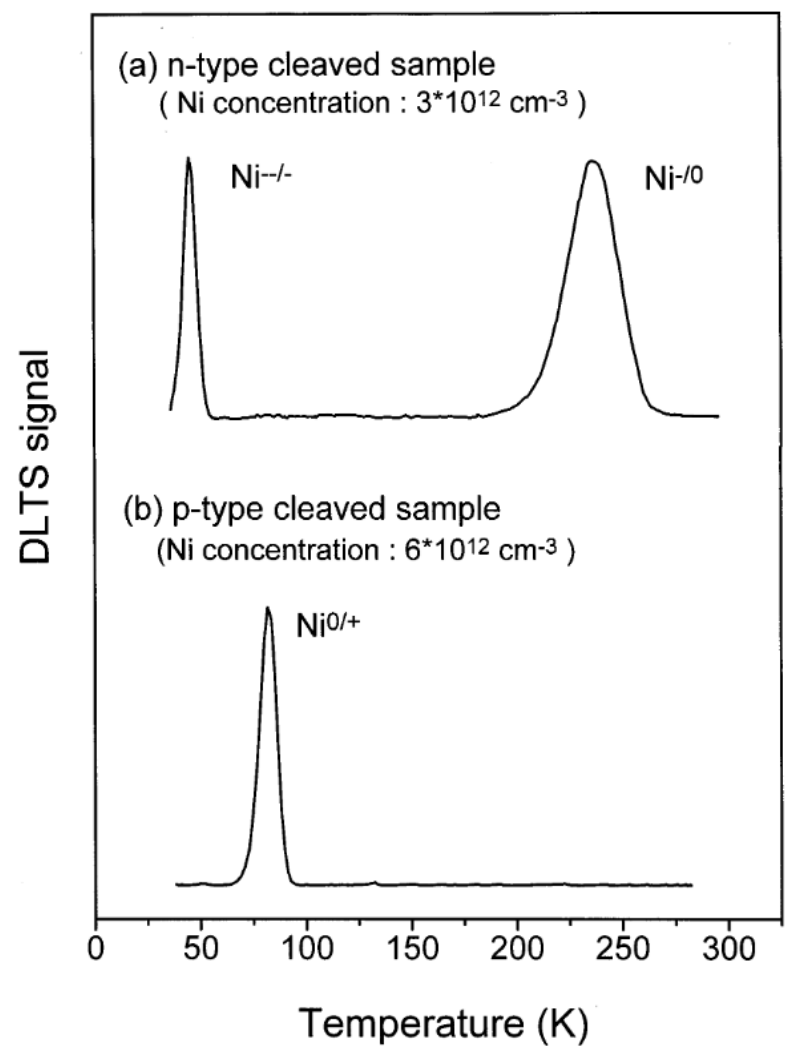

Figure 1.2: DLTS-signal of substitutional nickel in n-type and in p-type silicon, revealing levels at $E_{c}-0.06 \mathrm{eV}\left(\mathrm{Ni}^{--}--\right), E_{c}-0.43 \mathrm{eV}\left(\mathrm{Ni}^{-/ 0}\right)$, including the thermal activation of the capture cross section) and $E_{v}+0.15 \mathrm{eV}\left(\mathrm{Ni}^{0 /+}\right)$. Taken from [18].

The acceptor state of Ec- $0.43 \mathrm{eV}$ is used in section 3.1 to detect substitutional nickel.

\subsubsection{Nickel in-diffusion for vacancy marker analyses}

The applicability of marker analyses bases on the efficient and distinctive marking of the probed species with marker atoms, including the efficient propagation of the marker species in the host medium, the favored interaction of the marker with the probed species and a confident detection of the marked species. The idea of vacancy marker analyses in silicon is based on the reaction of pre- 
existing vacancies $\mathrm{V}$ with foreign marker atoms $\mathrm{M}$ according to the reaction:

$$
M_{\text {interstitial }}+V \rightleftharpoons M_{\text {substitutional }} .
$$

The marker atoms are commonly introduced in a thermal procedure by indiffusion via interstitial sites from a defined silicide boundary phase and are subsequently detected on substitutional sites by electrical analysis. The pioneering marker experiments have been performed with gold and platinum [60, 26], which were known as stable substitutional atoms. Parasitic contributions of the kick-out mechanism are reported, limiting the range of processing conditions. Suitable in-diffusion temperatures were $730^{\circ} \mathrm{C}$ within a process time of 20 min. As an alternative marker species, nickel has been applied for vacancy detection by Khorosheva [19]. The temperature-dependent interstitial solubilities and diffusivities for nickel [3, 4], platinum [60] and gold [60] are shown in fig. 1.3. Whereas the mobilities for the three sorts are roughly comparable, the interstitial solubility of nickel is orders of magnitude higher than for platinum and gold. This means that with nickel higher in-diffusion fluxes can be achieved at lower temperatures, which eventually allows thermal procedures that are less invasive for the grown-in defect landscape.

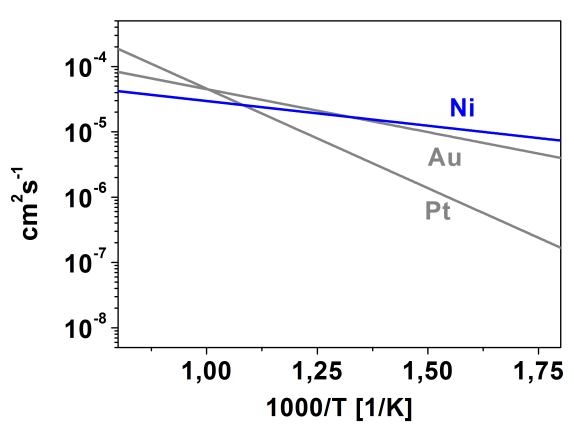

(a)

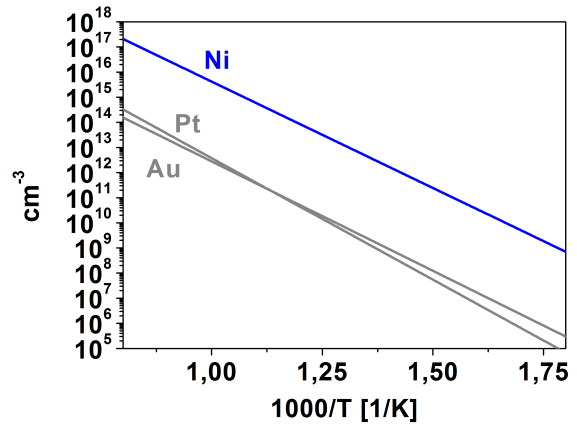

(b)

Figure 1.3: Arrheniusplots of the diffusivity (a) and the solubility (b) for interstitial gold, platinum and nickel. Whereas the mobilities for the three sorts are comparable, the interstitial solubility of nickel is orders of magnitude higher than for platinum and gold. The data for nickel are reported by Weber [3] and Lindroos et al. [4], the data for platinum are reported by Zimmermann and Ryssel [60].

The solubilities of the substitutional species are shown in fig. 1.4. The lower substitutional solubility of nickel atoms means a smaller tendency to bind to a vacancy, which lowers the contribution of kick-out mechanism and makes nickel marker in-diffusion particularly sensitive for the detection of weakly bound vacancies[61].

Exemplary diffusion lengths for typical conditions are depicted in fig. 1.5, with an assumed grown-in vacancy concentration of $[V]=10^{14} \mathrm{~cm}^{-3}$ and an 


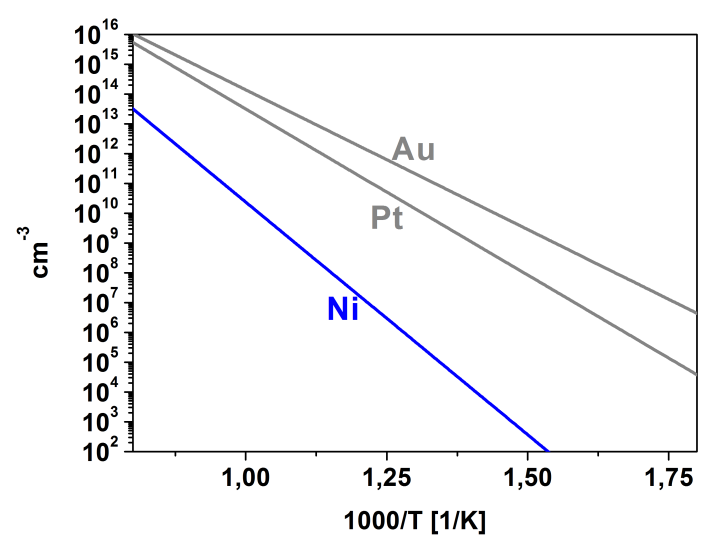

Figure 1.4: Arrheniusplots of the solubility of the substituional species of nickel, gold and platinum. The solubility of substitutional nickel is some orders of magnitude smaller than of substitutional platinum or gold. The data for nickel are reported by Yoshida and Saito [6], the data for platinum are reported by Zimmermann and Ryssel [60].

annealing time of $t=10 \mathrm{~min}$. For the underlying mechanism of trap-limited diffusion [62] the effective diffusion coefficient is given here by

$$
D_{e f f}=D \frac{\left[M_{i}\right]_{e q}}{[V]_{\text {init }}}
$$

with the indices 'eq' for the equilibrium concentration and 'init' for the initial concentration. It can be seen that the diffusion length of nickel is about two orders of magnitude above the diffusion lengths of gold or platinum. Even at $500{ }^{\circ} \mathrm{C}$ the diffusion length of nickel exceeds typical sample thicknesses $d_{\text {wafer }}$ below $500 \mu \mathrm{m}$.

Finally, the substitutional nickel can be identified by its acceptor state at $E c-0.43 \mathrm{eV}$. 


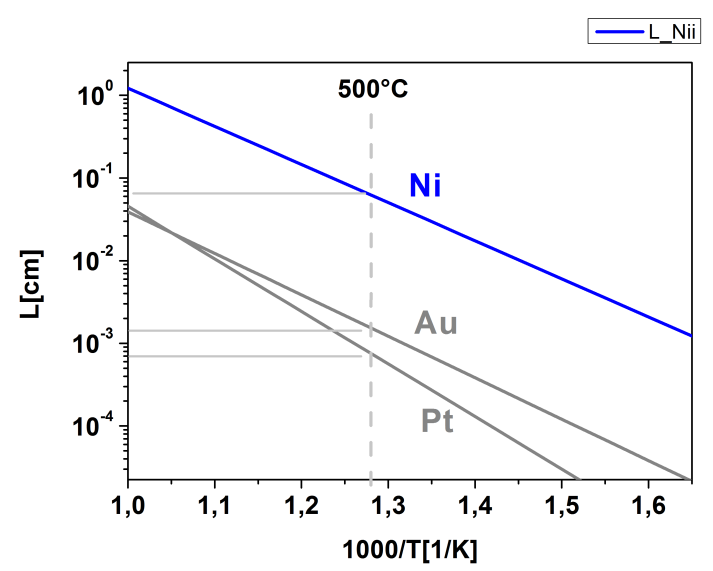

Figure 1.5: Arrheniusplots of the diffusion length for trap limited diffusion with an grown-in vacancy concentration of $[V]=10^{14} \mathrm{~cm}^{-3}$ and a typical in-diffusion time of 10 minutes. The diffusion length of nickel is nearly two orders of magnitude above the diffusion length of gold and platinum. Already at $T=500{ }^{\circ} \mathrm{C}$ the diffusion length of nickel exceeds typical wafer thicknesses $d_{\text {wafer }}<500 \mu \mathrm{m}$. 


\section{$1.2 \quad$ Nickel disilicide precipitation}

\subsubsection{Early stages of nickel disilicide precipitation}

Due to steeply decreasing solubility of interstitial nickel [3] in silicon with decreasing temperatures, rapid cooling of a silicon crystal, saturated during an in-diffusion anneal enables non-equilibrium conditions with rather high driving chemical potentials for precipitation. The precipitation of nickel in silicon in the sub-retrograde temperature regime under these supersaturation levels was first investigated by Seibt [63], finding evidence for the incorporation of nickel atoms into disc-shaped metastable $\mathrm{NiSi}_{2}$ particles via core sites of a geometrically necessary dislocation loop bounding the platelets on $\{111\}$-planes with a Burgers vector $b=a / 4<111>$. This precipitation mechanism was found to be decisive, in spite of the related interfacial and dislocation energies at the two dimensional structures, as it is assumed to maximize the energy degradation rate of the system. In the as-quenched state, the platelets have a thickness of only two monolayers. The atomic structure of the precipitates is illustrated in fig. 1.6. Both $\mathrm{NiSi}_{2} / \mathrm{Si}$-interfaces are formed by $\mathrm{Si}-\mathrm{Si}$ bonds. In the following, the term 'rapid quenching' or 'quenching' will be used in the sense of 'rapid cooling'. Please note that during these processes nickel atoms on interstitial sites are mobile.

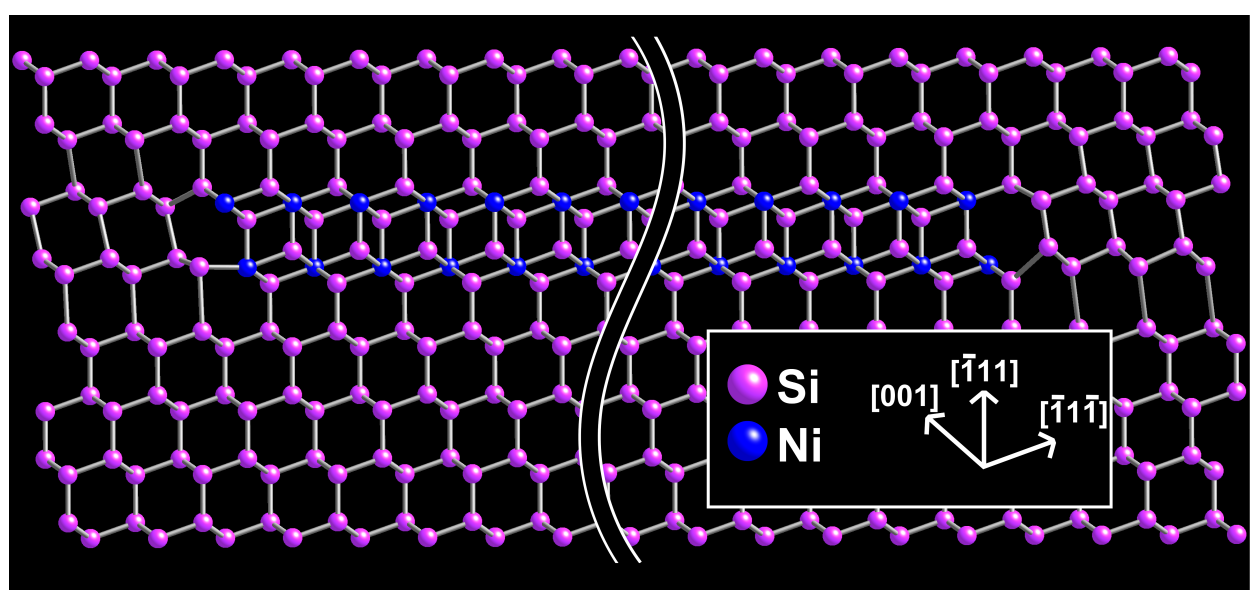

Figure 1.6: (110)-projection with the atomic structure of a two monolayer thick $\mathrm{NiSi}_{2}$ platelet, embedded in silicon matrix. The $\mathrm{NiSi}_{2}$-layers are coherent with $\mathrm{Si}(111)$ planes. Formation of Si-Si-bonds at both precipitate-matrix-interfaces leads to a sevenfold coordination of the nickel atoms and to a change in stacking sequence by a lattice displacement of $\mathrm{b}=\mathrm{a} / 4[111]$. In this structure, each nickel atom is in the interface, which is surrounded by a bounding dislocation. 


\subsubsection{Initial stages}

Calculations of the Gibbs free energy of circular $\mathrm{NiSi}_{2}$-platelets, which is the energy difference from assuming the same amount of involved nickel atoms in a silicide boundary layer in thermal equilibrium, are presented in detail e.g. by Riedel [10]. Contributions of the free energy result from the interface, $G_{G F}$, the strain energy $G_{S}$ and the bounding dislocation, $G_{R V}$, according to

$$
G=G_{G F}+G_{S}+G_{R V}
$$

For certain stages during cooling, the critical radius was estimated in Seibt and Schröter [9] by $r_{\text {crit }}=6 \mathrm{~nm}$ at $T=0.7 \times T_{\text {indiff }}$ or $r_{\text {crit }}=3 \mathrm{~nm}$ for the supersaturation at $T=0.5 \times T_{\text {indiff }}$, with energy values given in [64] and [65]. Extrapolating the stacking fault energy to initial stages would result in nucleation energies of the order of $100 \mathrm{eV}$. As was pointed out by Seibt and Schröter [9] for small precipitate radii, the incorporation of interstitial nickel atoms into the stacking fault structure requires stacking fault energies per atom, which far overbalance the driving chemical potential and make a stacking fault for the initial stages highly improbable. The question of early stages with a smaller nucleation barrier has not been answered yet and is still unexplored. In chapter 3.2 a reaction path for early nucleation stages is discussed. It is proposed that the nucleation barrier for the formation initial $\mathrm{NiSi}_{2}$ configurations could be significantly lowered with the involvement of substitutional nickel atoms.

\subsubsection{Nucleation processes}

The modelling of nucleation processes in the classical nucleation theory relies on the fundamental works by Volmer and Weber [66], Farkas [67], Becker and Döring [68]. The relevant aspects for the present study can be summarized as follows: In supersaturated systems, microscopic variations result in the volatile formation of clusters with the structure of the thermodynamic stable phase. If such embryonic state exceeds a critical nucleus size, it won't dissolve but further grows by aggregation of additional atoms to the equilibrium phase. In the picture employed here, we assume that the density of precipitates is linked with the nucleation rate $\mathrm{J}$, which is propertional to the concentration of nucleation sites $\mathrm{N}$ and a Boltzmann factor with nucleation barrier $E_{N B}$, according to

$$
J \propto N \times \exp \left(-\frac{E_{N B}}{k T}\right)
$$




\subsubsection{Determination of the precipitate density}

In earlier DLTS measurements of $\mathrm{NiSi}_{2}$ precipitates, described in more detail in section 1.3.1, Riedel [10] found a phenomenological correlation between the width of their DLTS signature and the precipitate diameter. The data are depicted in fig. 1.7. The width of the spectra is classified here by the Full Width Half Maximum (FWHM) at a pulse time $t_{p}=10 \mu \mathrm{s}$. In combination with the density of nickel atoms in $\mathrm{NiSi}_{2}$-phase and the concentration of precipitated nickel atoms according to the interstitial solubility at the in-diffusion temperature one can deduce the density of precipitates. In the analysis by Riedel [10] the precipitate densities were in the range of $N_{\text {prec }} 10^{12} \mathrm{~cm}^{-3}$ to $10^{13} \mathrm{~cm}^{-3}$. In the current work, the approach is used in section 3.2.1 to determine the precipitate densities from precipitation with and without available substitutional nickel.

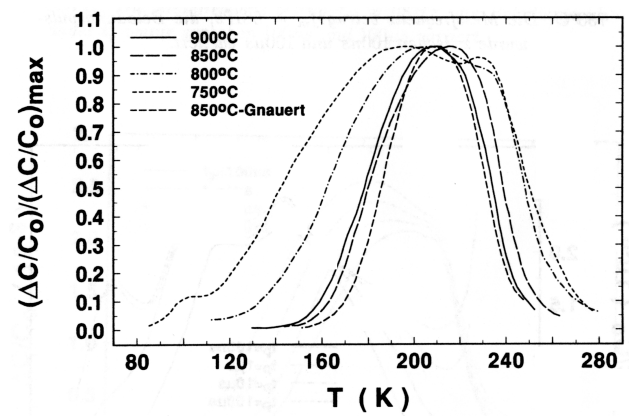

(a)

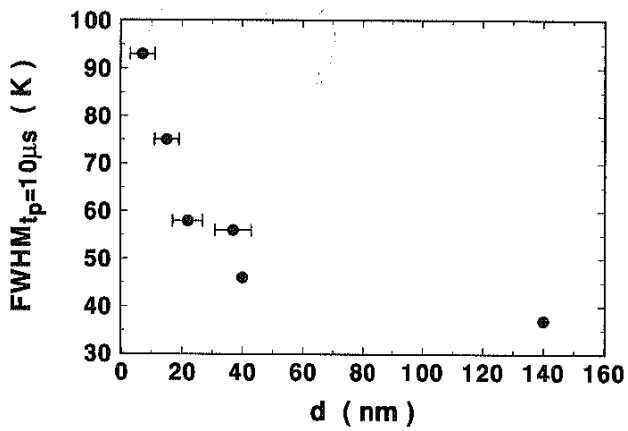

(b)

Figure 1.7: (a): Precipitate signals, measured by Riedel [10], for which the precipitate diameter was determined by Transmission Electron Microscopy. (b): Relation between the precipitate diameter and the FWHM of the DLTS signature. Both graphs are taken from Riedel [10]. 


\subsection{Electronic properties of $\mathrm{NiSi}_{2}$ precipitates}

\subsubsection{Previous DLTS analyses of $\mathrm{NiSi}_{2}$ precipitates}

The structural analysis of $\mathrm{NiSi}_{2}$ precipitates have been supplemented by the analysis of their electrical properties in DLTS-studies at large ensembles of precipitates by Gnauert [69], Riedel [10] and quotes therein, with first simulations by Kronewitz [70], refined by Hedemann [71]. A model of extended states with bandlike character was established, shown in fig. 1.8, according to an ensemble of deep multi-electron states with internal equilibration times $\Gamma_{i}$ shorter than capture or emission times $R^{-1}$.

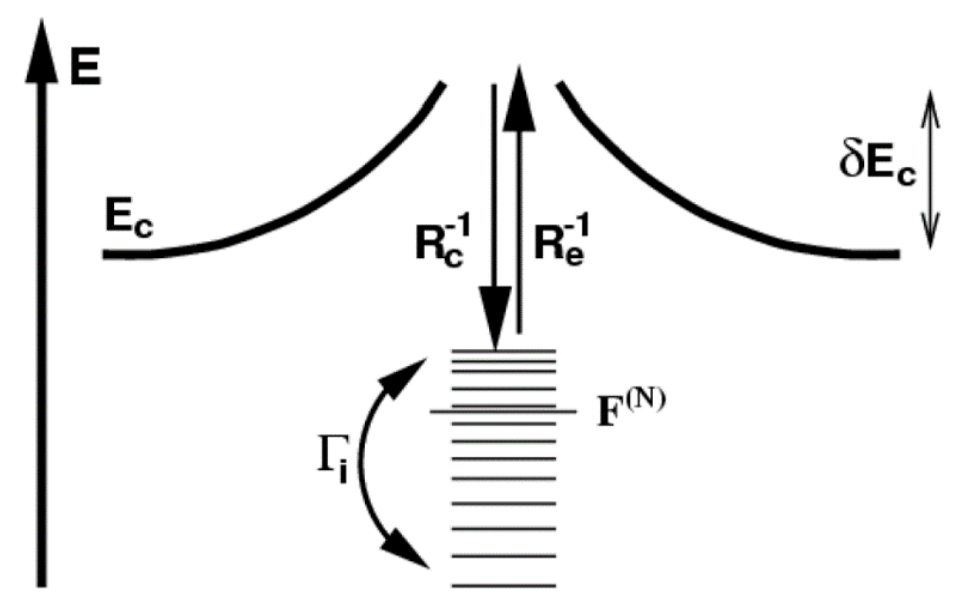

Figure 1.8: Schematic representation of the electronic model for $\mathrm{NiSi}_{2}$ precipitates, illustrating electron capture and emission at an extended defect: The electrostatic barrier $\delta E_{c}=e \Phi(F)$ with the total occupation $\mathrm{F}$ of defect states, electron capture and emission rates $R_{c}$ and $R_{e}$, and $\Gamma_{i}$ denoting the time constant for equilibration within the deep level system for fixed occupation. In the description of the system as extended defect with bandlike states, it must be $\Gamma_{i} \ll R_{e}^{-1}$. The occupation of the neutral defect is denoted by $F^{(N)}$ referred to as neutral occupation. Taken from Schröter et al. [72].

Typical DLTS-graphs of the precipitates with simulated profiles, in n-doped silicon, probing the upper half of the bandgap, are shown with the dotted curves in figs. 1.9 and 1.10. They are characterized by the extension of their low temperature side with increasing capture pulse durations. By convention, the spectra are classified by their Full Width Half Maximum (FWHM) at a pulse time $t_{p}=10 \mu \mathrm{s}$, which is related to the mean diameter of the corresponding precipitates. The numerical fits by Hedemann [71] comprehend a twodimensional distribution with rectangular density of states with $E=$ const $\left(E_{\min } \leq E \leq E_{\max }\right)$ and a onedimensional distribution with $E^{-1 / 2}$ singularities at the upper and lower energetic limit. According to fig.1.8 the model considers a total concentration of deep states at the precipitate $N_{T}$, a neutral- 
ity occupation $F_{N}$, i.e. the occupation for which the defect itself is neutral, a lower and an upper energetic limit of the defect band $\Delta H_{\text {min,max }}$, and a capture barrier $E_{\alpha}=e \Phi(F=1)$ under complete occupation (capture barrier coefficient). Pre-characterizing Hall measurements within the current work indicated a Fermi-level pinning around $E_{c}-0.3 \mathrm{eV}$ which is in accordance with the density of states, determined by Hedemann [71].

Whereas the electronic properties are well described in terms of bandlike states, the question concerning their origin appears rather complex. Remarkably, the spectra are confidently fitted with both, a 1D density of states $(D(E)=$ const $\left.\left(E_{\min } \leq E \leq E_{\max }\right)\right)$ in fig. 1.9 or with a $2 \mathrm{D}$ density of states $\left(D(E) \sim E^{-1 / 2}\right)$ as shown in fig. 1.10. Thus, the question if the states have predominantly 1Dcharacter, attributed to the unusual core structure of the bounding dislocation, or if they have 2D-character, attributed to the electronic structure with sevenfold coordinated nickel atoms at the interface, has not been definitely answered. In this view, a change in spectra in n-doped material under structural transformation during internal ripening with perturbations of the precipitate edge gave reason to assume that the bandlike extended states correspond to the bounding dislocation ring. A specific core structure was assumed with nickel atoms with deficient configuration [43].

Later DLTS and MCTS studies by Trushin et al. [73] indicated that the spectra in p-doped material remain unchanged during internal ripening, for which electronic states at the $\mathrm{NiSi}_{2} / \mathrm{Si}$-interface, constituting a defect band in the lower half of the bandgap, were proposed, as depicted in the model in fig. 1.11. This would eventually suggest that both, the interface and the bounding dislocation, are related to extended bandlike states. In this concept, the bandlike states of the bounding dislocation are largely in the upper half of the bandgap and the bandlike states of the interface are in the lower half of the bandgap. For more detailed insight, spatially resolved electrical measurements at individual structures are required, as will be presented in section 3.3. A sketch with a possible electronic model of the precipitates, combining deep states at the bounding dislocation, according to Riedel [43], shallow interface levels according to Schick [74] and bandlike interface states in the lower half of the bandgap, according to Trushin [73] is shown in fig.1.11.

\subsubsection{Recombination properties of $\mathrm{NiSi}_{2}$ precipitates}

Previous analyses of the recombination properties refer to $\mathrm{NiSi}_{2}$ precipitates, which had subjected to a ripening procedure. This had resulted [9] in particles with an increased thickness. In EBIC studies, Kittler et al. [75] found recombination contrasts of $\mathrm{c}=0.4$ at these precipitates, and identified the $\mathrm{NiSi}_{2}$ - precipitates as dominant recombination path in the samples. The high recombination activity was ascribed to the internal Schottky barriers at the 


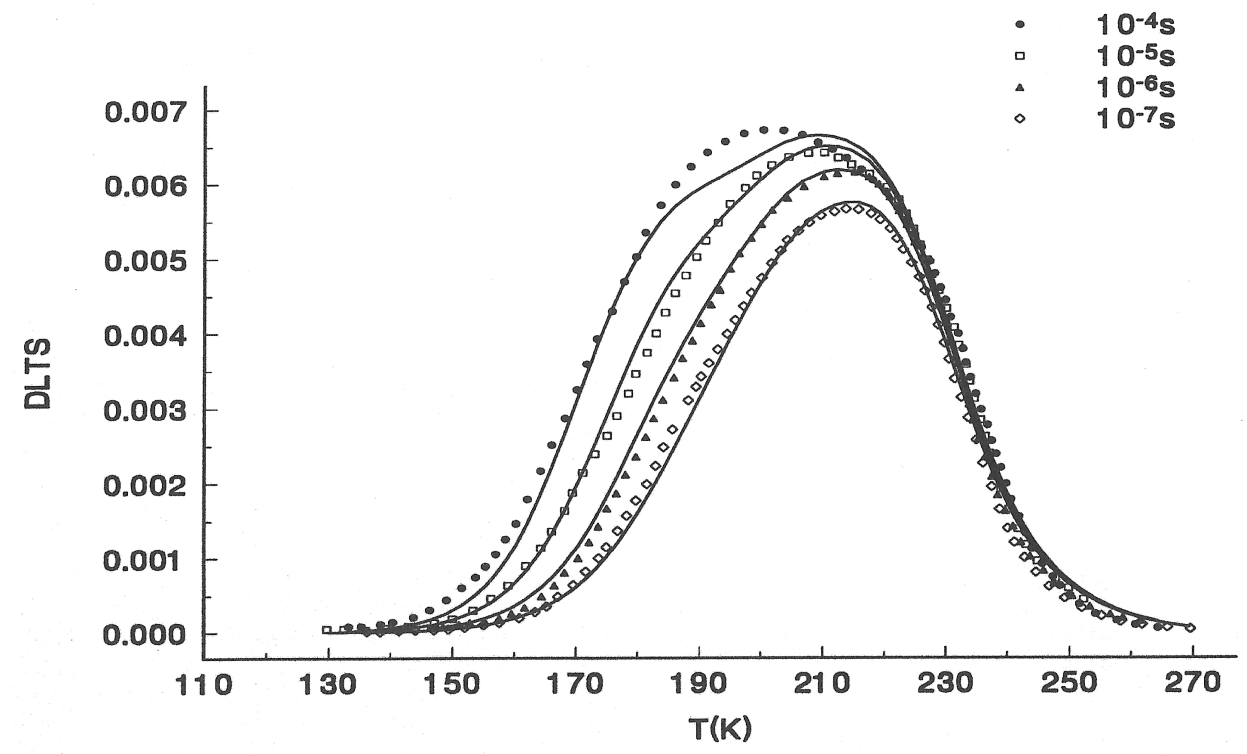

Figure 1.9: Measured DLTS signature (dotted) of $\mathrm{NiSi}_{2}$ precipitates after quenching from $900{ }^{\circ} \mathrm{C}$ with simulated curves (solid) on the basis of a onedimensional density of states, with a capture barrier coefficient $E_{\alpha}=0.47( \pm 0.01) \mathrm{eV}, \Delta H_{\min }=0.272 \mathrm{eV}$, $\Delta H_{\max }=0.419 \mathrm{eV}$ and neutrality level $F_{N}=0.44( \pm 0.05) \mathrm{eV}$, taken from Hedemann $[71]$.

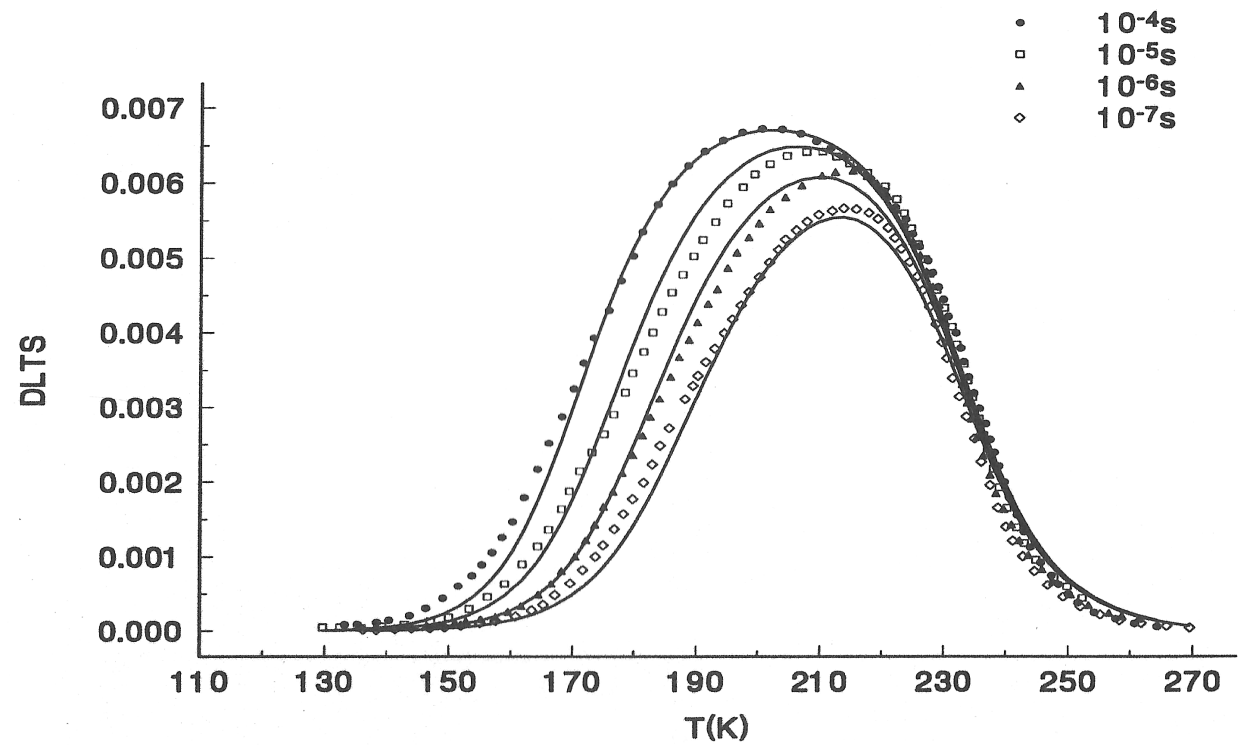

Figure 1.10: Measured DLTS signature (dotted) of $\mathrm{NiSi}_{2}$ precipitates after quenching from $900{ }^{\circ} \mathrm{C}$ with simulated curves (solid) on the basis of a twodimensional density of states, with a capture barrier coefficient $E_{\alpha}=0.48( \pm 0.03) \mathrm{eV}, \Delta H_{\min }=0.212 \mathrm{eV}$, $\Delta H_{\max }=0.452 \mathrm{eV}$ and neutrality level $F_{N}=0.27( \pm 0.02) \mathrm{eV}$, taken from Hedemann $[71]$. 


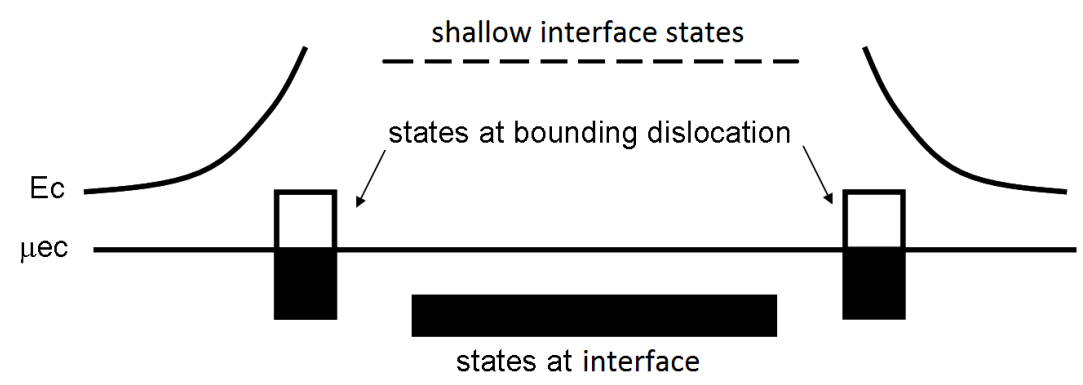

Figure 1.11: Possible band diagram for $\mathrm{NiSi}_{2}$ precipitates which contains bandlike states at the bounding dislocation, according to Riedel [43], shallow interface levels according to Schick [74], and bandlike states in the lower half of the bandgap, according to Trushin [73].

precipitates. An electron holography study by Formanek and Kittler [76] revealed direct evidence for a Schottky barrier with a height under illumination - affected by excess carriers - of $90 \mathrm{meV}$ from which they concluded a dark barrier of $0.3 \mathrm{eV}-0.4 \mathrm{eV}$. A strong recombination activity of $\mathrm{NiSi}_{2}$ precipitates indicates a Schottky barrier already in the thin as-quenched state, which was found within a pre-characterization in the current work with corresponding bulk diffusion lengths around $L=15 \mu \mathrm{m}$. 


\section{Chapter 2}

\section{Experimental}

\subsection{Preparation process}

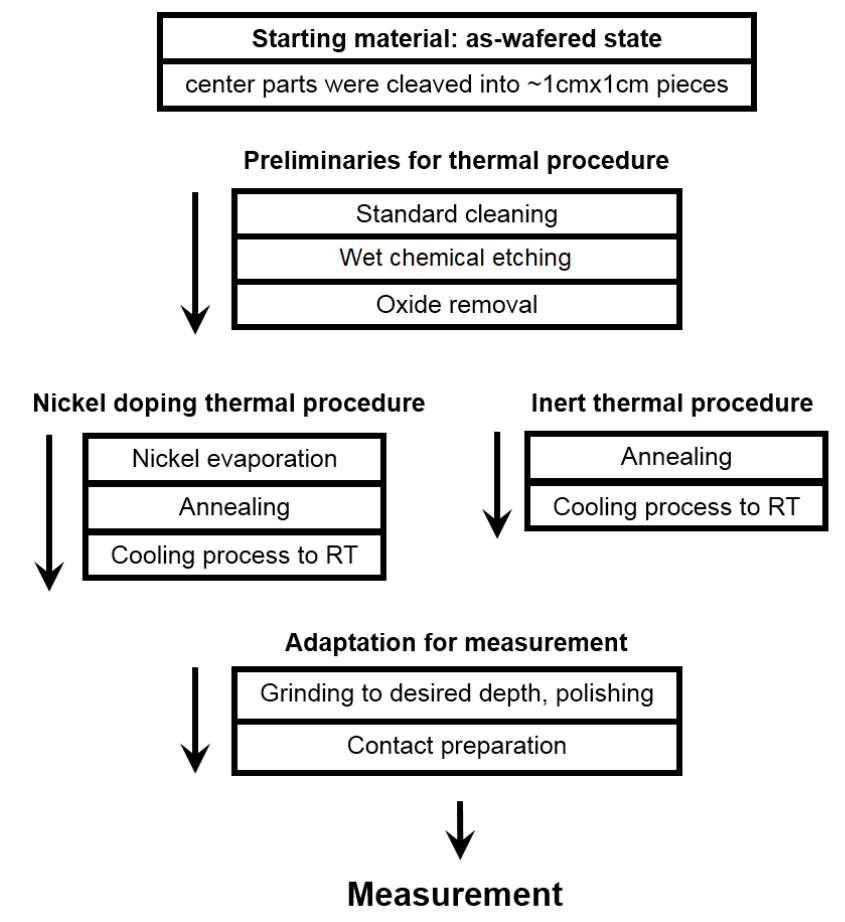

Figure 2.1: Block diagram of the sample preparation.

Fig. 2.1 gives an overview of the sample preparation procedures, applied within this work. The modular blocks are briefly described in the following; detailed information as e.g. about diffusion temperatures, cooling conditions are given in the respective respective context in chapter 3. 


\section{Starting material}

The starting material was out of as-wafered FZ-silicon samples, grown under vacancy-rich conditions in the standard industrial process by Wacker, with shallow doping concentrations of $3 \times 10^{14} \mathrm{~cm}^{-3}$ and $1 \times 10^{15} \mathrm{~cm}^{-3}$ in n-type material and $1 \times 10^{14} \mathrm{~cm}^{-3}$ in p-type material. The nitrogen concentration in each material was $0.5-3.5 \times 10^{15} \mathrm{~cm}^{-3}$ (information from Wacker). The oxygen concentration was $1-2 \times 10^{15} \mathrm{~cm}^{-3}$ (information from Wacker). To probe the relation between the grown-in defects and nitrogen, additional FZ-samples, provided by N. Abrosimov from the Institut für Kristallzucht (IKZ), were analyzed. These samples were out of one crystal doped with nitrogen and one crystal, grown in nitrogen-free ambient. Reference samples from other growth methods were Cz-silicon, provided by the MEMC Electronic Materials from Prof. V. V. Voronkov and Dr. R. Falster, and monocrystalline and multicrystalline block cast silicon, produced within the SolarWinS-project. The wafers were cleaved in $\approx 1 \mathrm{~cm} \times 1 \mathrm{~cm}$ pieces. The samples for the analysis were taken from center parts of the wafers.

\section{Preliminaries for annealing procedure}

Prior to the annealing procedures, the sample surfaces were cleaned with a standard cleaning procedure in deionized water $\left(3 \times 4 \mathrm{~min} \mathrm{H}_{2} \mathrm{O}\right)$ and organic solvents ( $5 \mathrm{~min}$ acetone, $5 \mathrm{~min}$ methanol) in an ultrasonic bath and finally brief rinsing in $\mathrm{H}_{2} \mathrm{O}$. Possible subsurface impurities, introduced in the wafering process, were removed by wet chemical etching with removal of ca 5 micrometers from the surfaces by a mixture of $\mathrm{HF}(4 \%)$ and $\mathrm{HNO}_{3}(44 \%)$. Subsequently, oxidized surface layers were removed by HF.

\section{Nickel doping procedure}

For nickel in-diffusion the samples were then immediately mounted in an ultra high vacuum chamber and nickel was thermally evaporated from one large side to a thickness of $20-30 \mathrm{~nm}$. Subsequently, the samples were inserted into the furnace. The respective annealing procedures were performed in tube furnaces with the samples in a quartz tube in inert atmosphere, realized by an argon flow through the quartz tube. For the different experiments, annealing temperature and time were varied, which will respectively be described in the context.

The cooling rate terminating the annealing process is decisive for the reaction path of the interstitial nickel. Sufficiently small cooling rates were chosen within the marker experiments, allowing the interstitial outdiffusion and segregation in the nickel silicide boundary phase. For this purpose the samples were 
put out of the quartz tube and brought into contact with an aluminum heat sink. The rate should not be too small to avoid the dissolution of substitutional nickel. Sufficiently large cooling rates were chosen within the precipitation experiments. Therefore precipitation was triggered by quenching in ethylene glycol with an estimated cooling rate of $1000 \mathrm{~K} / \mathrm{s}$ or in $\mathrm{NaOH}$, with an estimated quenching rate of $2000 \mathrm{~K} / \mathrm{s}$ [77]. Quenching after temperatures above $950{ }^{\circ} \mathrm{C}$ often resulted in sample breaking. Therefore, for higher temperatures only quenching in ethylene glycol was applicable.

\section{Inert annealing procedures}

For the annealing without nickel in-diffusion the mounting in the UHV chamber was skipped and the samples were immediately inserted into the furnace.

\section{Adaption for the measurement}

For subsequent measurements, the samples were ground so that the nickel disilicide boundary layer and the near surface regions were removed, standardly down to a depth of $95 \mu \mathrm{m}-105 \mu \mathrm{m}$ below the former boundary layer. Particular depth-dependent analyses were enabled by grinding bevel surfaces. The created surfaces were polished with diamond polishing paste down to optical flatness and finally chemomechanically polished with syton suspension. In a water-repellance test, the removal of oxide was proved.

Schottky contacts for DLTS measurements were produced by thermal evaporation of gold. During contact preparation after the last thermal procedure, wet chemical hydrogenative etching was avoided. This was essential in order to avoid parasitic nickel hydrogen reactions [18]. The effect of hydrogenative etching of our samples within contact preparation after nickel in-diffusion is illustrated in fig. 2.2. The additional lines after hydrogenative etching indicate nickel hydrogen complexes, which already formed at room temperature.

For the electrical measurements, Schottky-contacts were prepared with gold on n-doped silicon and aluminium on p-type silicon. For DLTS measurements, the contact thickness was about $200 \mathrm{~nm}$ and the diameter was $0.68 \mathrm{~mm}$. For LBIC and EBIC measurements the contact thickness was $15-20 \mathrm{~nm}$, and the contacts covered the whole front area. Ohmic contacts for Hall measurement were prepared by evaporation of $\mathrm{Al}$ on n-type on the corners of $5 \mathrm{~mm} \times 5 \mathrm{~mm}$ samples. For defect preferential etching, the evaporation of metal contacts was omitted. 

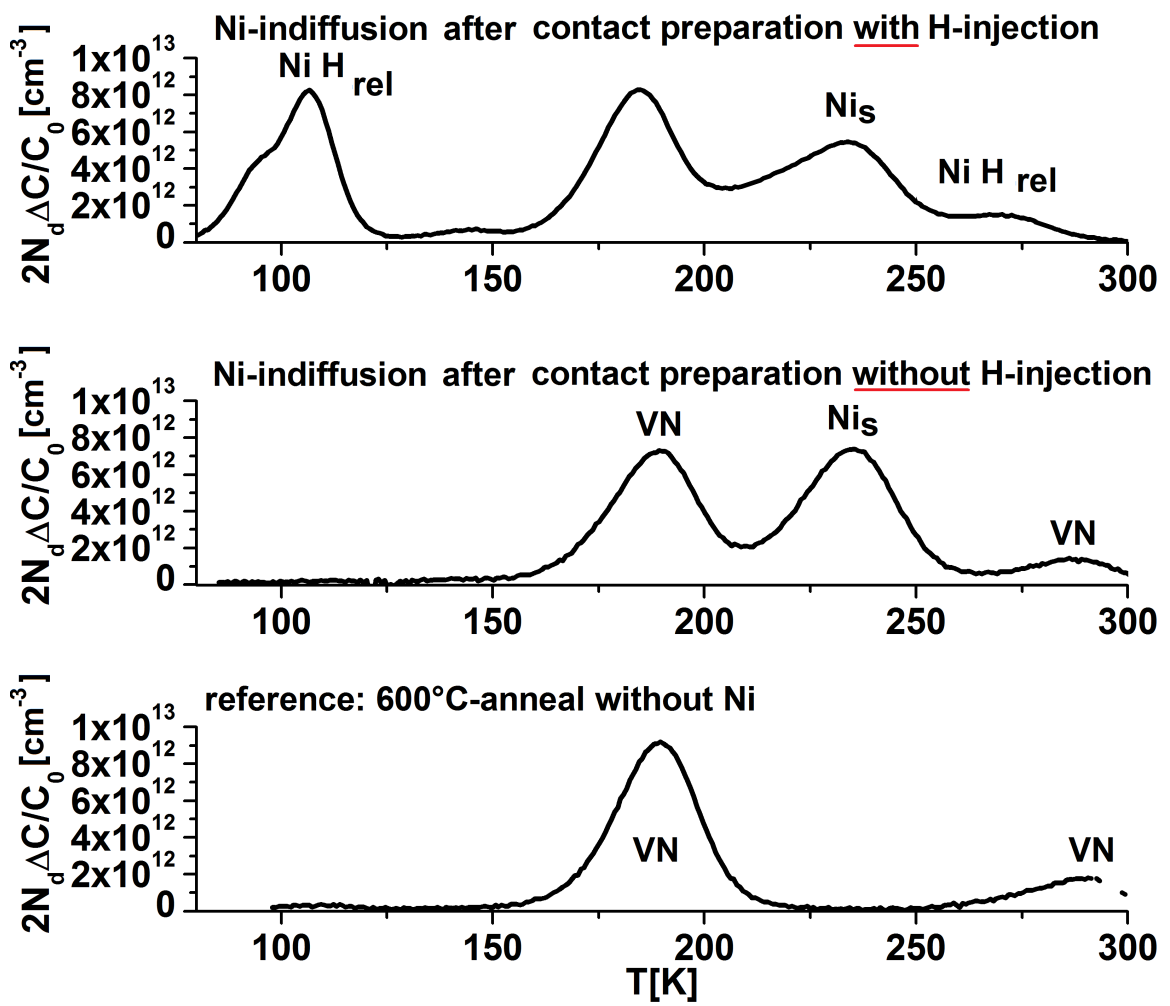

Figure 2.2: DLTS-graphs illustrating the effect of hydrogenative etching. The presented spectra are taken after nickel in-diffusion with hydrogenative etching (top), nickel in-diffusion without hydrogenative etching (center) and after reference annealing without nickel in-diffusion. In the case with hydrogenative etching, additionel deep levels (labelled with $\mathrm{Ni} \mathrm{H}$ rel) are observed, related to nickel hydrogen complexes. These additional defects were avoided without etching. The reference sample indicates additional deep levels which are presented in detail in sec. 3.1 


\subsection{Deep Level Transient Spectroscopy (DLTS)}

\subsubsection{Method}

The DLTS method, developed by Lang [78], enables efficient analysis of deep levels at crystal defects in semiconductors. It is based on the measurement of the capacitance response from capture and emission processes of deep levels in the space charge region of a diode structure on an applied periodic voltage modulation. During the capture period $t_{p}$, a voltage pulse $U_{p}$ that is superimposed on a constant reverse bias voltage $U_{0}$, pumps the space charge region and rises the occupation of the deep levels there. During the subsequent emission period, the relaxation to the equilibrium under $U=U_{0}$ is probed by analysis of the corresponding change of capacitance $\Delta C$. A detailed review of the method is given e.g. by Blood and Orton, [79] which is briefly summarized in the following.

For a single type of deep levels - with overall concentration $N_{T}$, the concentration of occupied levels $n_{T}$ and the concentration of electrons $n$ in the conduction band and holes $p$ in the valence band - charge processes can be described in form of the rate equation

$$
\frac{\mathrm{d} n_{T}}{\mathrm{~d} t}=\left(c_{n} n+e_{p}\right)\left(N_{T}-n_{T}\right)-\left(e_{n}+c_{p} p\right) n_{T},
$$

$c_{i}$ and $e_{i}$ are temperature-dependent capture coefficients and emission rates for electrons (index $\mathrm{n}$ ) and holes (index $\mathrm{p}$ ). It is the general case - assuming the interaction with both charge types, electrons and holes - which will be referred to in section 3.1.1 within the specific analysis of a near midgap level. The usual case is the interaction with one charge type. For deep levels in the upper half of the band gap in an n-type semiconductor, one approximates

$$
\frac{\mathrm{d} n_{T}}{\mathrm{~d} t}=c_{n} n\left(N_{T}-n_{T}\right)-e_{n} n_{T}
$$

For the emission period $\left(c_{n} n=0\right)$ one obtains

$$
n_{T}(t)=n_{T}(0) \cdot \exp \left(-e_{n} t\right)
$$

Within certain limits [79] the occupation $n_{T}(t)$ is proportional to the capacitance signal $\Delta C(t)=C(t)-C_{0}\left(C_{0}=\right.$ capacitance at the end of the emission 
period under reverse bias $U_{0}$ ). Assuming that the deep levels of the whole space charge region are involved in the charge processes, one further obtains as a rough approximation

$$
\begin{aligned}
\Delta C(t) & =\frac{C_{0}}{2 \cdot N_{d}} \cdot n_{T}(t) \\
\Delta C(t) & =\Delta C \cdot \exp \left(-e_{n} t\right) \\
\text { with } \Delta C & =\frac{C_{0}}{2 \cdot N_{d}} \cdot N_{T} .
\end{aligned}
$$

A more accurate expression for $\Delta C$ (eq.2.6), derived by Pons [80], includes the dependence on the detailed conditions of the filling process. The corresponding analytical equations, used in the present work are presented in the appendix A.2.

From historically established context, the capacitance transients are evaluated by correlation of the capacitance transient with a periodic function and temperature-scanning. For a sine correlation, one obtains a spectrum $\mathrm{S}(\mathrm{T})$, according to

$$
S(T)=f_{\text {corr }} \int_{t_{g}}^{t_{g}+f_{\text {corr }}^{-1}} \Delta C \cdot \exp \left(-e_{n}(T) t\right) \cdot \sin \left(2 \pi f_{\text {corr }} t\right) d t
$$

with the correlation frequency $f_{\text {corr }}$. The gate time $t_{g}<<f_{\text {corr }}^{-1}$ is necessary to blank the measurement device-specific setup process after the filling pulse. It is usually small and can be neglected.

A theoretical spectrum is shown in fig 2.3. In the maximum, one obtains the relations $f_{\text {corr }}=\alpha \cdot e_{n}$ with $\alpha=0.424$ and $\mathrm{S}=\Delta C \cdot \beta$ with $\beta=0.126$.

The position of the maximum shifts with different correlation frequencies, which allows the analysis of $e_{n}(T)$ according to

$$
\begin{aligned}
e_{n}(T) & =\sigma_{n} \chi_{n}<v_{t h}>N_{c} \exp \left(-\frac{\Delta H_{n}}{k_{B} T}\right) \\
& =\gamma \sigma_{n} \chi_{n} T^{2} \exp \left(-\frac{\Delta H_{n}}{k_{B} T}\right)
\end{aligned}
$$




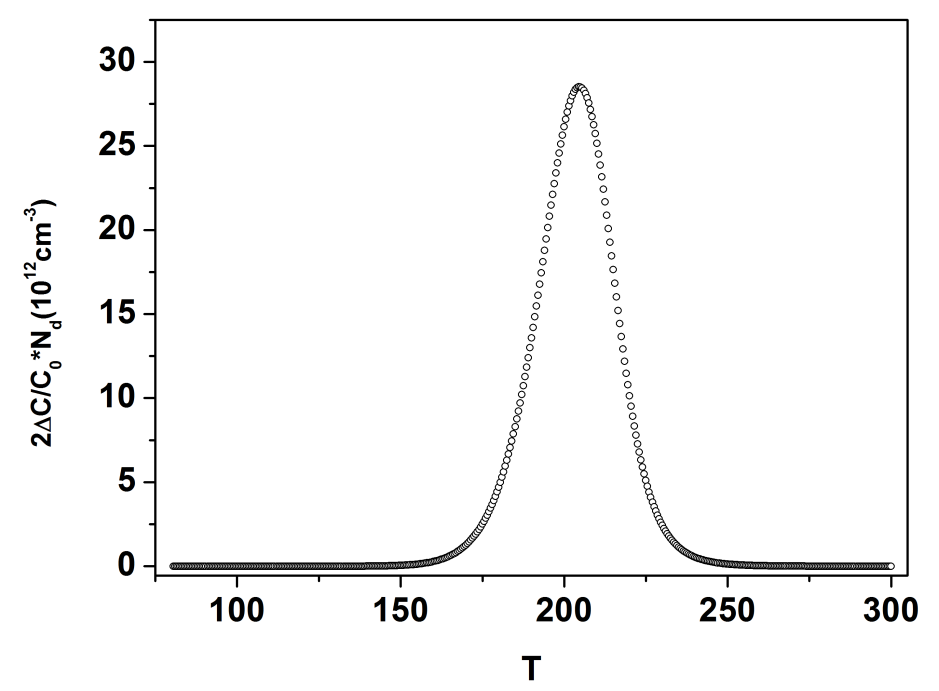

Figure 2.3: Simulated DLTS-graph of an isolated point defect.

$\left(\chi_{n}=\exp \left(-\Delta S_{n} / k_{B}\right), \gamma_{T}^{2}=<v>_{n} N_{c}\right)$ from which the activation enthalpy

$$
\Delta H_{n}=-k_{B} \frac{d \ln \left(\frac{e_{n}(T)}{T^{2}}\right)}{d\left(\frac{1}{T}\right)}
$$

for the electron emission in the conduction band can be determined. The cross section and an entropy factor with all related entropy contributions of the emission process are included in

$$
\ln \left(\gamma \sigma_{n} \chi_{n}\right)=\left.\frac{\ln \left(e_{n}\right)}{T^{2}}\right|_{T \rightarrow \infty} .
$$

In combination with the analysis of the capture cross section by Pons, the entropy factor can be determined.

\subsubsection{DLTS measurement details}

The DLTS analyses were performed with a setup, that has been arranged within the diploma thesis of Oliver Voss [81]. Concentrations of the deep levels have been derived from the DLTS line amplitude, taking into account 
Pons corrections for capture in the Debye tail and for large trap concentrations [80]. Standard parameters for DLTS measurements were a bias voltage during emission period $V_{r}=-5 \mathrm{~V}$, pulse voltage $V_{p}=-0.2 \mathrm{~V}$ and pulse duration $t_{p}=100 \mu \mathrm{s}$. If not otherwise indicated, the spectra were obtained from $17 \mathrm{~Hz}$ sine correlation, where the temperature of the line maximum - with underlying exponential emission - corresponds to an emission rate of $e_{p}=40.1 \mathrm{~s}^{-1}$.

\subsection{Electron Beam-Induced Current (EBIC)}

The Electron Beam Induced Current-method, e.g. reviewed in Leamy [82], is a powerful scanning technique for the spatial analysis of recombination active features in the vicinity of a charge collecting contact. Electrons and holes, pairwise generated by an incident electron beam, either recombine at internal bandgap states of crystal defects or are separated by a rectifying contact and contribute to an external current.

According to [83], the generation rate $g(r, z, R)$ in a depth $z$ and a lateral distance $r$ from the beam axis, depending on the primary electron beam parameters is given by

$$
g(r, z ; R)=\frac{A(z)}{2 \pi \sigma(z)} \cdot \exp \left(-\frac{r^{2}}{2 \sigma^{2}(z)}\right)
$$

$A(z)$ represents the depth-dose function

$$
A(z)=\frac{g_{0}}{R} \Lambda(z / R)
$$

with the polynomial parametrization [84] for $\Lambda$ in silicon

$$
\begin{aligned}
& \Lambda(\xi)=0.6+6.21 \xi-12.4 \xi^{2}+5.69 x^{3} ; 0 \leq \xi \leq 1.1 \\
& \Lambda(\xi)=0 \quad \xi \geq 1.1
\end{aligned}
$$

and

$$
\sigma^{2}(z, R)=0.11 z^{3} / R
$$

and an empirical expression for R: 


$$
R / \mu \mathrm{m}=0.017 \cdot\left(E_{\text {beam }} / \mathrm{kV}\right)^{1.75}
$$

The total generation rate $g_{0}$ is given by

$$
g_{0}=\frac{E_{\text {beam }} I_{\text {beam }} \cdot(1-f)}{E_{e-h} q} .
$$

Therein, $f$ is the backscattered fraction of the beam energy and can be estimated with $f=0.08$. The energy expended to the formation of an electron hole pair is $E_{e-h}=3.8 \mathrm{eV}$ for silicon. The present EBIC analysis was performed with a standard GATAN EBIC setup with a Stanford SR570 current amplifier. The width of the incident electron beam is focused below $20 \mathrm{~nm}$, as was determined from SEM micrographs. The electron energies ranged from $2 \mathrm{kV}$ to $30 \mathrm{kV}$. The depth extension of the probing volume was estimated with the primary electron range $R[\mu \mathrm{m}]=0.017 \cdot E[\mathrm{kV}]^{1.75}$, which is $60 \mathrm{~nm}$ for $2 \mathrm{kV}$ and $6.54 \mu \mathrm{m}$ for $30 \mathrm{kV}$. The broadening of the generation volume due to lateral scattering of the incident electrons $\sigma^{2}$ in a depth $z$ is estimated by means of the expression $\sigma^{2}=0.11 \cdot z^{3} / R([83])$. Generation was performed under low injection conditions.

\subsection{Preparation for EBIC analyses of precipitates}

To access the precipitates in preparation for EBIC measurements, samples have been ground, by which the former nickel layer and about $200 \mu \mathrm{m}$ of the regions below have been removed. After polishing with a grain size from $15 \mu \mathrm{m}$ down to $0.25 \mu \mathrm{m}$ and cleaning in $\mathrm{H}_{2} \mathrm{O}$, in acetone and in methanol, a thin gold layer contact has been evaporated as a Schottky emitter contact on the whole area of the the front surface. Previously, wet chemical etching was observed to preferentially corrode $\mathrm{NiSi}_{2}$-structures at the surface (L. Stolze, private communication, 2009). By skipping wet chemical etching here, the preparation of the Schottky contact deviated from the standard procedure (section 2.1) to preserve the $\mathrm{NiSi}_{2}$ structures attaching the surface. A second, low-ohmic contact has been installed by scratching a gallium/aluminum alloy on the silicon backside. 


\subsection{Focused Ion Beam (FIB) preparation details for cross section EBIC and Transmission Electron Microscopy (TEM)}

For subsequent Transmission Electron Microscopy (TEM) analysis, cross section samples were carved out with Focused Ion Beam from FEI Nova NanoLab 600, making use of the in-situ EBIC FIB technique [85]. For this purpose, the adjacent material was removed by the use of gallium ions, starting with $30 \mathrm{kV}$ and $7 \mathrm{nA}$ for rough milling of the material and finishing with $5 \mathrm{kV}$ and $29 \mathrm{pA}$. The regions on top of the structure were preserved by preceding deposition of a platinum and carbon containing composite (Pt:C). Details of the TEM analysis are described in the respective context. 


\section{Chapter 3}

\section{Results and discussion}

\subsection{Kinetics of grown-in vacancy-related complexes in FZ-silicon and their interaction with nickel atoms}

Recently, silicon from the float zone growth process, ensuring the highest 'purity', has been described [25] to degrade during inert annealing while deep band gap levels appear in significant concentrations. Earlier studies suggest $[86,24,87]$ that these phenomena are associated with nitrogen-related grownin complexes, which transform during subsequent thermal processes. However a unique picture of the underlying complexes and their kinetics is still missing.

In the present study, the annealing behavior of the grown-in complexes is systematically examined in silicon wafer material in temperature-dependent analyses. Subsequent to the characterization of the principal deep levels (section 3.1.1), the involvement of weakly bound vacancies in the grown-in complexes is figured out by nickel marker in-diffusion (section 3.1.2). The annealing properties are gathered in an annealing scheme for nitrogen-vacancy-related complexes (section 3.1.3). The absence of weakly bound vacancies in 'impure' silicon materials is discussed in the context of additional agents serving as prevalent sinks (section 3.1.4). This will lead to an evaluation of the nickel marker diffusion method (section 3.1.5).

If not otherwise indicated in the text, our analyses have been conducted at samples out of FZ-silicon wafers from Wacker. Complementary experiments with material, provided by the Institut für Kristallzucht, Berlin, imply that the observations concerning the nitrogen vacancy complex species and their evolution upon annealing can be generalized to FZ-silicon of the current commercial standard, crystallized under fast pull rates with vacancy-rich conditions. 


\subsubsection{Deep bandgap levels}

Principal results of DLTS measurements in n-type FZ-silicon, probing the upper half of the bandgap, are presented in fig. 3.1. The nitrogen concentration was in the range of (see section 1.1.1) $C_{N t o t}=1 \times 10^{15} \mathrm{~cm}^{-3}$. Deep levels are detected after inert annealing (solid curve) in the medium temperature range between $500^{\circ} \mathrm{C}$ and $800^{\circ} \mathrm{C}$. Among them, two levels, which are denoted by X186 and X295, systematically appear. The absence of these levels in as-grown samples as well as in reference samples (dashed curves), grown in nitrogen-free ambient, implies that the observed levels form during the annealing of a native nitrogen-related complex. The level at $T=110 \mathrm{~K}$, tentatively associated with the A center (whose properties are e.g. reviewed in Pichler [54]), appears irregularly and is not relevant for the further analysis. Deep level spectra by Grant et al. [25] revealed additional defects that do not appear in our spectra. We believe that this may be due to their annealing in dry oxygen, whereas we have used inert argon gas.

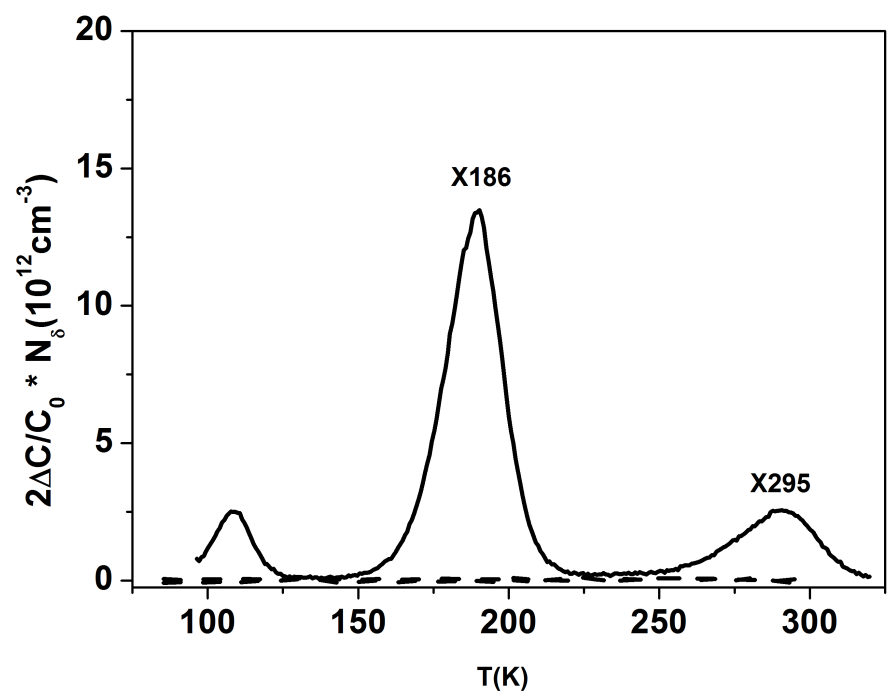

Figure 3.1: DLTS graphs of n-type FZ-silicon. Deep levels are observed after annealing (here at $550^{\circ} \mathrm{C}$ ) in the standard material from the industrial FZ-silicon growth process (solid curve) under vacancy-rich conditions with nitrogen concentrations of $C_{N t o t}=1 \times 10^{15} \mathrm{~cm}^{-3}$. Two levels, denoted by X186 and X295 have been systematically observed. In as-grown material and in nitrogen-free reference material (dashed curves) the levels are absent. 


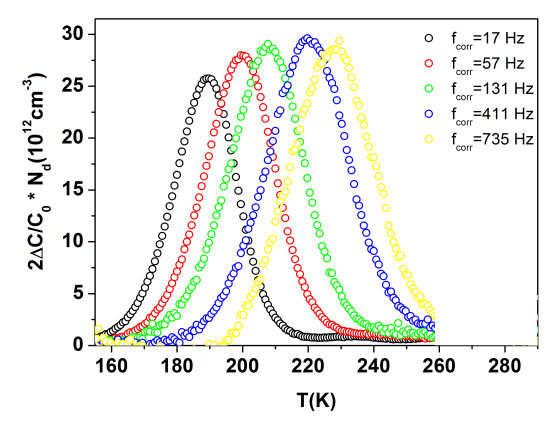

(a)

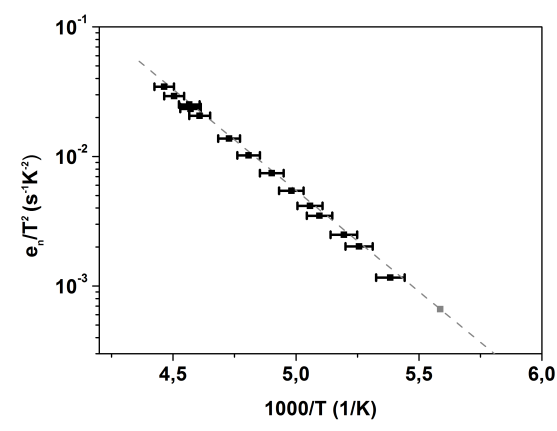

(b)

Figure 3.2: DLTS analysis of the thermal emission at the X186-level. (a) Exemplary DLTS graphs with different correlation frequencies $f_{\text {corr }} / \mathrm{Hz}=17,57,131,411,735$. (b) Arrhenius plot of the emission coefficient (squares). The signature coincides with an earlier described level (dashed gray line), which was related to substitutional nitrogen [88].

The thermal emission characteristics of the X186-level, shown in fig. 3.2 reveals an electron activation enthalpy of $\Delta H=0.32 \mathrm{eV}$. This corresponds to the principal level found in DLTS analyses by Murakami et al. [88] after introduction of nitrogen by $\mathrm{N}_{2}^{+}$-ion implantation and pulsed laser annealing. Murakami et al. interpreted this level as substitutional nitrogen.

Its capture kinetics, which we obtained from variation of the DLTS filling pulse length, are depicted in fig. 3.3. The line graph in fig. 3.3(b) is obtained from a least squares fit of the Pons-corrected (see appendix A.2) exponential capture kinetics to the experimental data. With a mean thermal electron velocity of $[89]$

$$
<v_{t h}>[\mathrm{cm} / \mathrm{s}]=1.17 \cdot 10^{6} \sqrt{T[\mathrm{~K}]}
$$

the resulting capture cross section is $2.6 \cdot 10^{-17} \mathrm{~cm}^{-2}$. A different value of $\sigma=1 \times 10^{-15} \mathrm{~cm}^{2}$ had been derived by Murakami et al. [88] in Electron Spin Resonance (ESR) analysis. They pointed out that their determination of the capture cross section from the ESR-spectra assumes the present level to be the only band gap level of the underlying complex. However, this is not applicable, as will become clear from our following findings.

A peculiarity can be found from comparing the amplitudes of X186 and X295, as illustrated in fig. 3.4. In all analyzed samples, independent of the shallow doping concentration, through different annealing temperatures and annealing times, the ratio of the Pons-corrected amplitudes of X186/X295 is revealed to be constant. In accordance to analogous findings by Utzig and Schröter [91] for different charge states of substitutional gold, we conclude that the present 


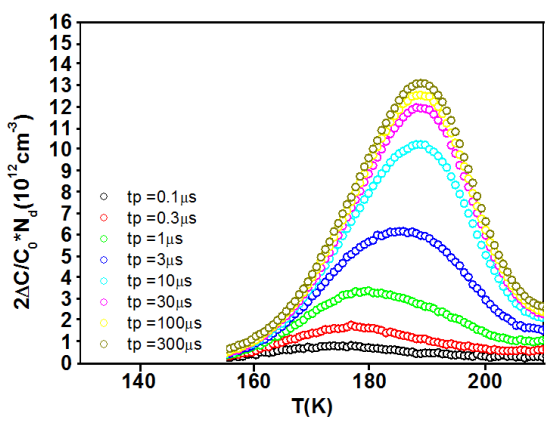

(a)

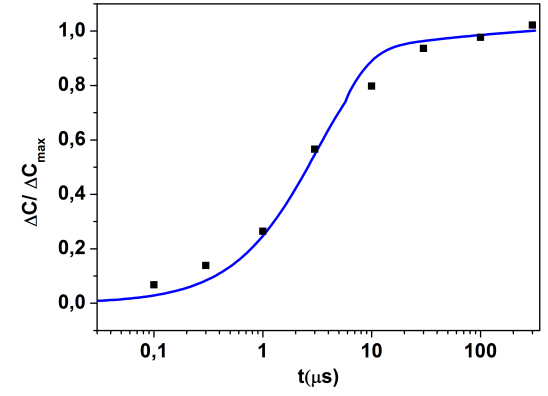

(b)

Figure 3.3: DLTS capture kinetics of the level X186. (a) DLTS graphs with pulse durations $t p / \mu \mathrm{s}=0.1,0.3,1,3,10,30,100,300$. The signature is overlapped by a second point defect, causing a slight maximum shift at small pulse durations. However, due to its much smaller concentration, the additional point defect can be neglected here. The data points were obtained in the Bachelor thesis of Ahrens [90]. (b) Pons-corrected fit (solid line) of the capture pulse dependence, revealing a capture cross section of $\sigma_{n}=2.6 \cdot 10^{-17} \mathrm{~cm}^{-2}$.

levels are two levels of one complex.

\section{Analysis of the X295 near midgap level}

The smaller amplitude of the X295 level (than of the X186 level) matches the finding that the X295 level is detected in p-type material, as well. It indicates that both majority and minority carriers are involved in the emission processes. Typical spectra of the X295 level in both doping types, with selected correlation frequencies, are illustrated in fig. 3.5. It becomes obvious that the respective temperatures of the maxima are the same in n-type and p-type material. The corresponding emission rates for n-type and p-type samples are plotted in one Arrhenius diagram in fig. 3.6(a). Comparing the amplitudes of 3.5(a) with 3.5(b) reveals a counteractive trend, which is expressed as a temperature-dependent amplitude ratio, plotted in fig. 3.6(b).

For a detailed evaluation, the following approach encompasses the bipolar character of the X295 level as a basis to separate the unipolar emission parameters for electrons (index $\mathrm{n}$ ) and holes (index p). According to the corresponding emission rate equation, e.g. in Blood and Orton [79], equation 7.84, the overall emission process of the near midgap level with the situation of balancing majority and minority carrier emission is governed by

$$
\frac{\mathrm{d} N(t)}{\mathrm{d} t}=e_{p}\left(N_{T}-N(t)\right)-e_{n} N(t)
$$




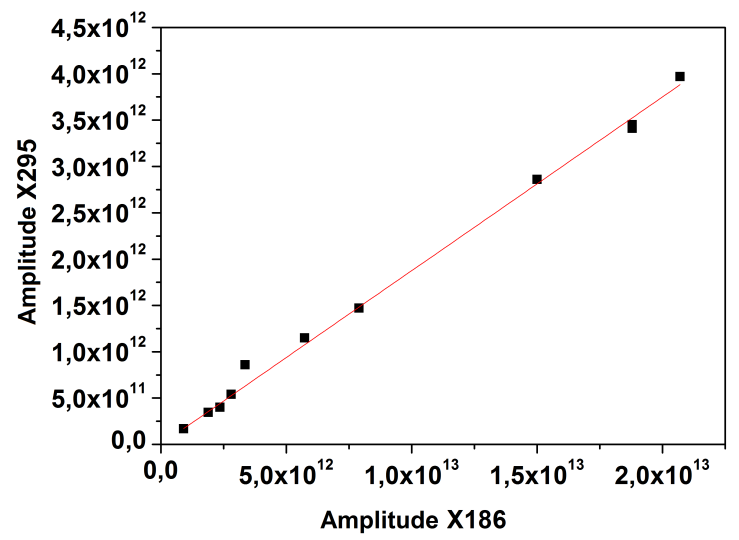

Figure 3.4: Plot of the DLTS amplitude of X295 in n-type material versus X186. Independent of shallow doping concentration or annealing temperature, the levels occur in all samples in the same ratio $r$. The linear fit (red line) reveals $r=$ $0.1875( \pm 0.0025)$.

$N_{T}$ is the total concentration of the deep levels, $N(t)$ the occupied portion and $e_{n, p}$ the emission rates for electrons and holes with the expressions

$$
e_{n}=A_{n} T^{2} \cdot \exp \left(\frac{-\Delta H_{n}}{k_{B} T}\right) \text { and } e_{p}=A_{p} T^{2} \cdot \exp \left(\frac{-\Delta H_{p}}{k_{B} T}\right)
$$

and the Boltzmann constant $k_{B}$ therein. In these equations, the only unknown parameters to be determined are the activation enthalpies $\Delta H_{n, p}$ and the prefactors $A_{n, p}$. For this purpose, the first order linear differential equation 3.2 is solved in the form

$$
N(t)=\frac{e_{p}}{e_{n}+e_{p}} N_{T}+\left(\frac{N(0)}{N_{T}}-\frac{e_{p}}{e_{n}+e_{p}}\right) \cdot \exp \left(-\left(e_{n}+e_{p}\right) t\right) N_{T}
$$

with $N(0)=N_{T}$ in n-type and $N(0)=0$ in p-type. It follows that the occupation converges for both doping types towards

$$
N_{\infty}=\frac{e_{p}}{e_{n}+e_{p}} N_{T}
$$

Compared to strict majority carrier emission, the DLTS amplitudes are thus scaled by the factors $\frac{e_{n}}{e_{n}+e_{p}}$ in n-type and $\frac{e_{p}}{e_{n}+e_{p}}$ in p-type. Using equation 3.3 , their ratio can be written in the following temperature-dependent form, according to an Arrhenius law

$$
\ln \left(e_{p} / e_{n}\right)\left(T^{-1}\right)=\ln \left(\frac{A_{p}}{A_{n}}\right)+\frac{\Delta H_{n}-\Delta H_{p}}{k_{B}} \cdot \frac{1}{T} .
$$




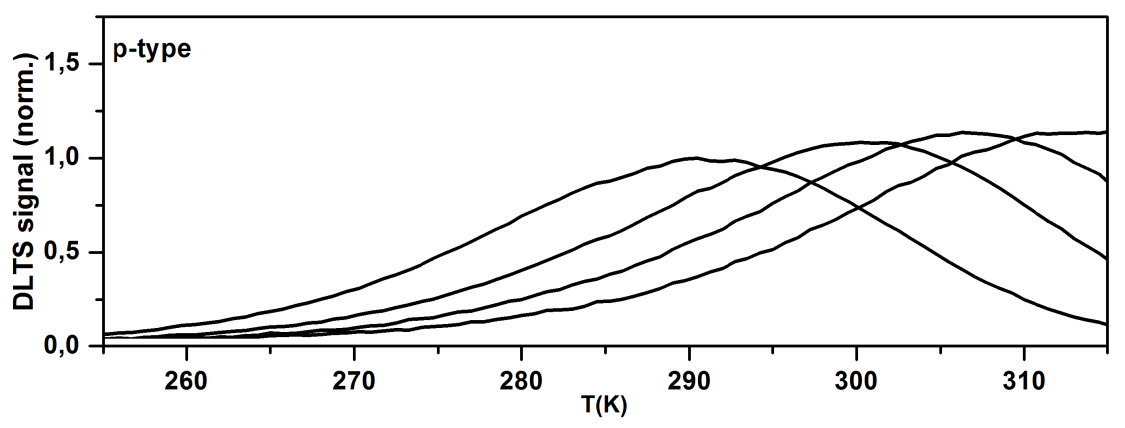

(a)

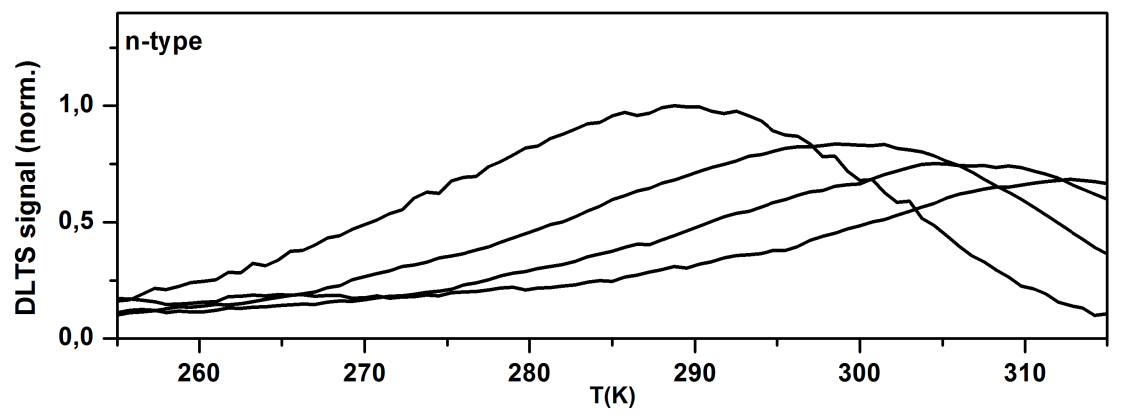

(b)

Figure 3.5: DLTS-signature of the X295 near midgap level in p-type (a) and n-type material (b) for the selected correlation frequencies (respectively from left to right) $f_{\text {corr }} / \mathrm{Hz}=17,39,73,131$. The temperature of the maxima is the same in both cases.

A least squares linear fit to the experimental data for the temperature-dependent amplitude ratio in fig. 3.6(b) reveals the slope

$$
k_{B} \cdot m_{A r r 1}:=k_{B} \cdot \frac{d \ln \left(e_{p} / e_{n}\right)}{d(1 / T)}=\Delta H_{n}-\Delta H_{p}=-0.17( \pm 0.2)
$$

and the ordinate intercept

$$
\Delta y_{A r r 1}:=\left.\ln \left(e_{p} / e_{p}\right)\right|_{\left(T^{-1}\right)=0}=\ln \left(\frac{A_{p}}{A_{n}}\right)=8.0( \pm 0.3)
$$

with the Pearson correlation coefficient $R=-0.99463$. 


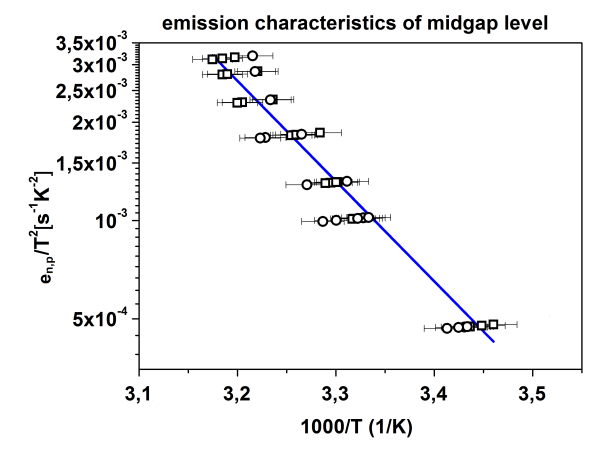

(a)

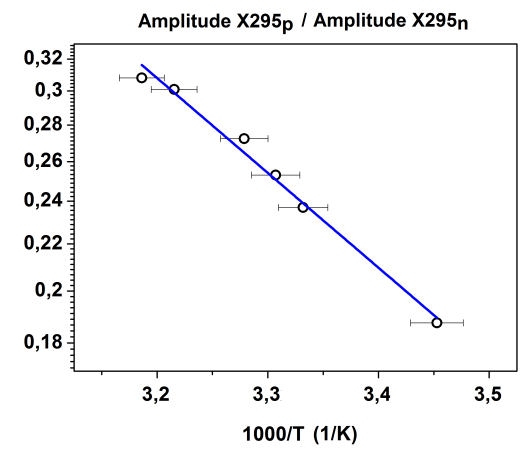

(b)

Figure 3.6: (a) Arrheniusplot of the thermal emission rates in n-type (squares) and in p-type (circles), plotted in one graph. (b) Arrheniusplot of the amplitude ratio for selected pairs in p-type and n-type. According to eq. 3.6 and following, the relations are used to derive the unipolar emission parameters, summarized in table 3.1 .

For the total emission rate (eq. 3.3) one obtains the relation

$$
\ln \left(\frac{e_{n}+e_{p}}{T^{2}}\right)=\ln \left(A_{n} \cdot \exp \left(-\frac{\Delta H_{n}}{k_{B} T}\right)+A_{p} \cdot \exp \left(-\frac{\Delta H_{p}}{k_{B} T}\right)\right)
$$

By inserting $\Delta H_{p}=\Delta H_{n}-m_{A r r 1} \cdot k_{B}$ from eq. 3.7 and $A_{p}=A_{n} \cdot \exp \left(\Delta y_{A r r 1}\right)$ from eq. 3.8 in eq. 3.9 , one obtains

$$
\begin{aligned}
\ln \left(\frac{e_{n}+e_{p}}{T^{2}}\right)=\ln & \left(A_{n} \exp \left(-\frac{\Delta H_{n}}{k_{B} T}\right)\right. \\
& \left.+A_{n} \exp \left(\Delta y_{A r r 1}\right) \exp \left(\frac{m_{A r r 1} k_{B}-\Delta H_{n}}{k_{B} T}\right)\right) .
\end{aligned}
$$

A least squares fit to the experimental data, presented in fig. 3.6 (a), with the variables $A_{n}$ and $\Delta H_{n}$ finally enables to determine all parameters $A_{n}, \Delta H_{n}$, $A_{p}$, and $\Delta H_{p}$. They are summarized in table 3.1. The correlation coefficient for the linear fit is $\mathrm{R}=-0.9743$.

The capture kinetics for electrons and holes were obtained from respective variation of the DLTS filling pulse length, depicted in figs. 3.7 and 3.8. The respective line graphs are obtained from a least squares fit of the Pons-corrected exponential capture kinetics to the experimental data. With a thermal electron velocity according to eq. 3.1 one obtains the capture cross sections for electrons and holes, $\sigma_{n}=4.4 \cdot 10^{-18} \mathrm{~cm}^{-2}$ and $\sigma_{p}=3.8 \cdot 10^{-16} \mathrm{~cm}^{-2}$. The capture cross sections are summarized in table 3.1 . 


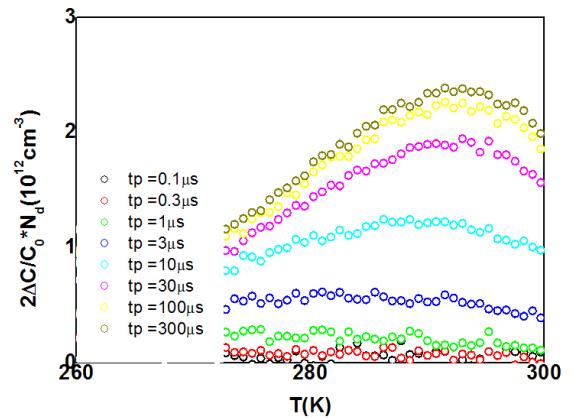

(a)

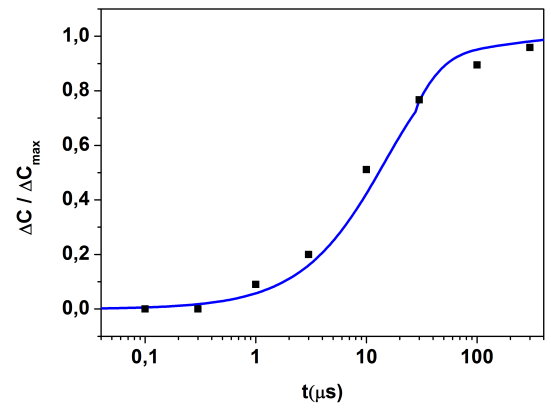

(b)

Figure 3.7: (a) DLTS graphs of the X295 level in n-type material with capture pulse durations of $t_{p} / \mu \mathrm{s}=0.1,0.3,1,3,10,30,100,300$. The datapoints were obtained within the Bachelor thesis of Ahrens [90]. (b) Normalized electron capture kinetics of the level X295 and Pons-corrected fit (solid line).

\begin{tabular}{|c|c|c|c|c|}
\hline level & $\Delta H_{n, p}[\mathrm{eV}]$ & $\sigma_{n, p}\left[\mathrm{~cm}^{-2}\right]$ & $A_{n, p}\left[s^{-1}\right]$ & $\Delta S_{n, p} / k$ \\
\hline $\mathrm{X} 186(\mathrm{n})$ & $0.32(2)$ & $2.6 \cdot 10^{-17}$ & $0.846 \cdot 10^{6}$ & 1.6 \\
$\mathrm{X} 295(\mathrm{n})$ & $0.5(2)$ & $4.4 \cdot 10^{-18}$ & $6.8( \pm 5.6) \cdot 10^{4}$ & 0.41 \\
$\mathrm{X} 295(\mathrm{p})$ & $0.66(3)$ & $3.8 \cdot 10^{-16}$ & $9.77( \pm 8.5) \cdot 10^{7}$ & 4.4 \\
\hline
\end{tabular}

Table 3.1: Activation enthalpy $\Delta H_{n, p}$, capture cross section $\sigma_{n, p}$, prefactor $A_{n, p}$ of the thermal emission and entropy $S_{n, p}$ of the nitrogen-related band gap levels. The indices (n) and (p) designate values for electrons or holes, respectively. The capture cross section was determined by the Pons-corrected analysis of the capture kinetics from the variation of the filling pulse length. The expression of the entropy was obtained from $\Delta S_{n, p} / k=\frac{A_{n, p} \cdot(T / \mathrm{K})^{2}}{\sigma_{n, p}<v_{t h}>N_{c}}$.

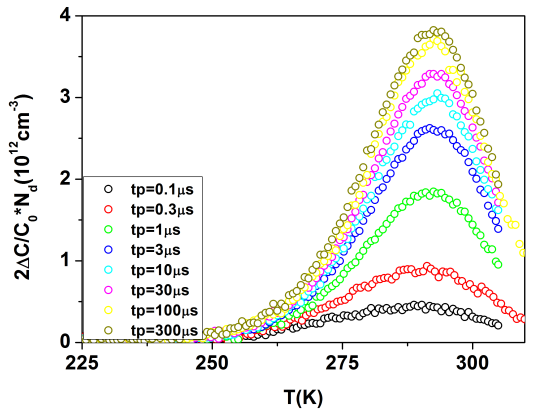

(a)

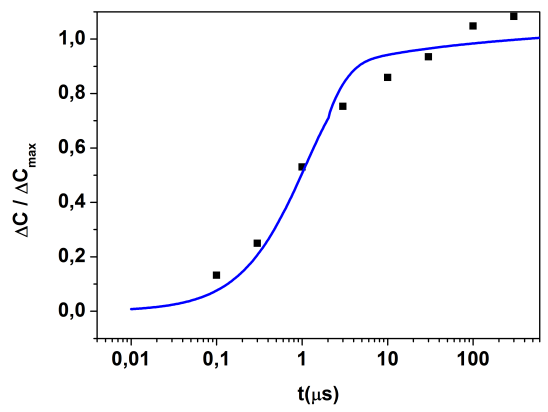

(b)

Figure 3.8: (a) DLTS graphs of the X295 level in p-type material with capture pulse durations of $t_{p} / \mu \mathrm{s}=0.1,0.3,1,3,10,30,100,300$. The datapoints were obtained within the Bachelor thesis of Ahrens [90]. (b) Normalized hole capture kinetics of the level X295 and Pons-corrected fit (solid line). 


\section{Summary}

The annealing of nitrogen doped vacancy-rich FZ-silicon results in a formation of deep levels, attributed to the conversion of the grown-in nitrogen-related complexes. In addition to an already known deep level (labelled here with X186) related to substitutional nitrogen, a second level (X295) of the same complex is found near the midgap. The capture and emission parameters of the levels are summarized in table 3.1.

\subsubsection{Vacancies tracked with nickel in-diffusion}

The involvement of vacancies in the medium temperature annealing reactions is explored by making use of their interaction with nickel. As described in section 2.1 nickel is supplied from a thermally evaporated boundary layer at one large surface of the sample. Due to rather low formation energies, interstitial nickel atoms penetrate the crystal already under a rather low thermal budget, and are available to be trapped at weakly bound vacancies and form substitutional nickel.

Fig. 3.9 shows DLTS graphs after nickel in-diffusion at medium temperatures in FZ-samples, grown without and with nitrogen. Obviously, substitutional nickel (labelled with $\mathrm{Ni}_{\mathrm{s}}$ ) forms only in the nitrogen-doped material, fig. 3.9(b), which illustrates that the native nitrogen complexes in FZ-silicon are associated with a severe amount of weakly bound vacancies.

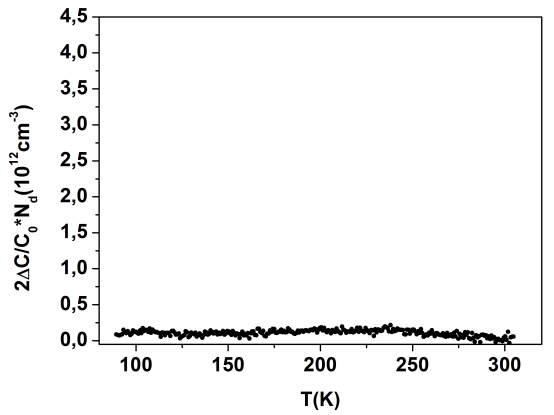

(a)

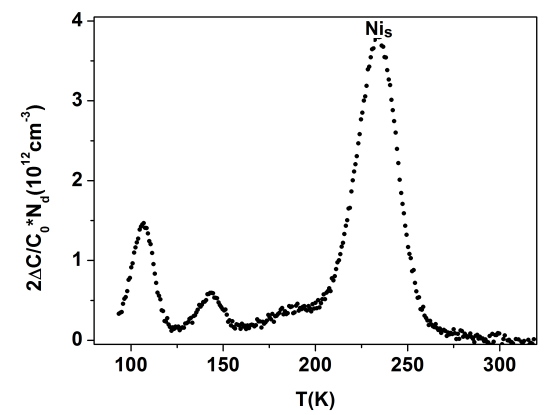

(b)

Figure 3.9: DLTS graphs after nickel in-diffusion at $680^{\circ} \mathrm{C}$ for $10 \mathrm{~min}$ in silicon samples grown without and with nitrogen. Whereas no deep levels are detected in the nitrogen-free material (a), the spectra in the nitrogen-containing material (b) exhibit a predominant signal around $T=230 \mathrm{~K}$, attributed to the deep acceptor state at $E_{c}-0.43 \mathrm{eV}$ of substitutional nickel. The findings imply a connection between the nitrogen complex family and high vacancy concentrations. 
The concentration of detected vacancies varied in different samples. In wafers from Wacker, maximum concentrations of $3 \cdot 10^{14} \mathrm{~cm}^{-3}$ are found with nickel in-diffusion into as-grown material and into samples after low pre-annealing temperatures. These concentrations coincide with the expected excess vacancy concentration from the difference in incorporated concentrations of $\mathrm{V}$ and $\mathrm{I}$ at the crystallization front that is supposed to remain after Frenkel recombination [20]. Even higher vacancy concentrations in the low $10^{15} \mathrm{~cm}^{-3}$-range have been found earlier in FZ-silicon by Abe [92], bound in divacancies that are incorporated during the crystal growth. In our samples which comply with the current commercial standard, divacancy bandgap levels are absent in the as-grown state.

\section{Summary}

Nickel in-diffusion indicates a high concentration of grown-in weakly bound vacancies in nitrogen-doped material. Maximum detected vacancy concentrations are in the range of the difference in incorporated concentrations of $\mathrm{V}$ and I at the melting temperature.

\subsubsection{Annealing stages}

The systematic analysis of the deep level spectrum over a wide temperature range, accompanied by nickel marker in-diffusion, establishes a picture of the annealing conversions of the grown-in complexes that is illustrated in the nitrogen-vacancy-annealing scheme for float zone silicon in fig. 3.10. Three annealing regimes are distinguished, which can be outlined as follows:

I The initial defect landscape includes a remarkably high concentration of volatile, electrically inactive complexes, related to vacancies and nitrogen atoms, maintained below $450^{\circ} \mathrm{C}$.

II Transformation of the initial complexes along with an electrical activation starts around $500{ }^{\circ} \mathrm{C}$, probably via intermediate states, by multiple release of vacancies. These get lost or are absorbed by a stable sink and the nitrogen-vacancy-related X186 complex with two deep bandgap levels emerges.

III Irreversible annealing of the X186 complex occurs above $650^{\circ} \mathrm{C}$, probably by decomposition and further release of a vacancy. 


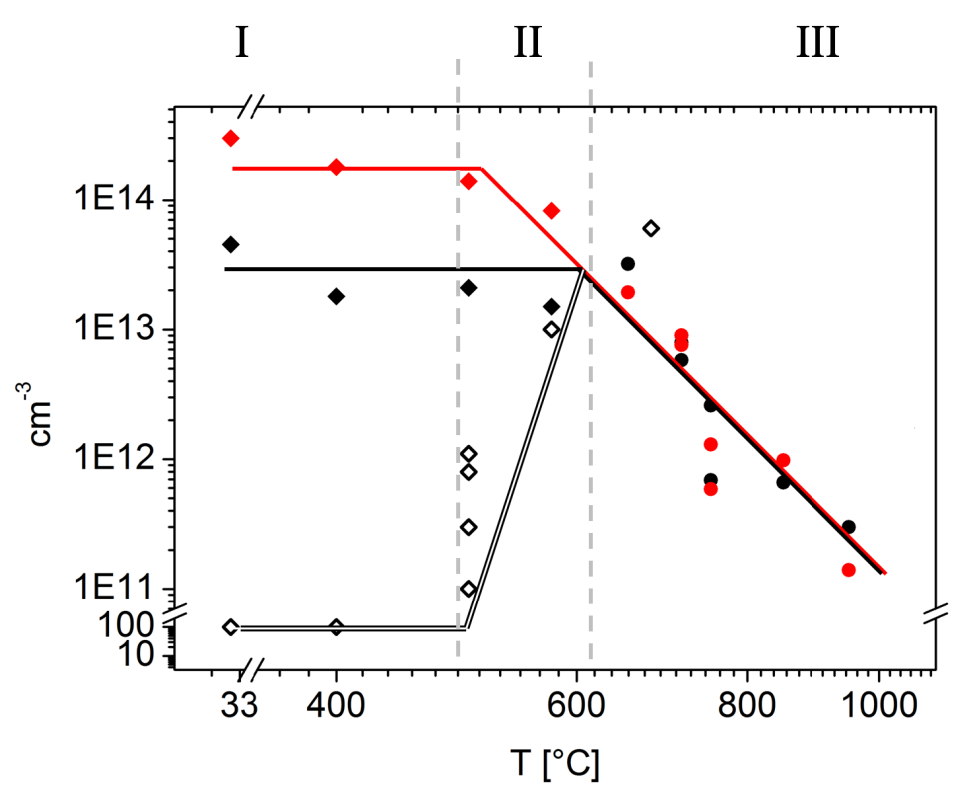

Figure 3.10: Annealing scheme of grown-in vacancy-nitrogen-complexes in FZsilicon, showing the concentration of substitutional nickel, formed at weakly bound vacancies (red line), and concentration of the X186 complex (filled black line), from nickel in-diffusion after different pre-annealing temperatures. The concentration of the X186 complex directly after pre-annealing is represented by the open black line. Circles represent datapoints, measured by Ahrens [90]. 


\section{Initial stage}

Identical DLTS graphs were obtained over the range from the as-grown state to annealing for $10 \mathrm{~min}$ at $400^{\circ} \mathrm{C}$. Figure 3.11 exemplarily shows the absence of the nitrogen complex after low temperature inert annealing. The concentration of vacancies, detected by nickel in-diffusion, is virtually unaffected by the lowtemperature procedures which implies that the initial complexes are stable in this range.

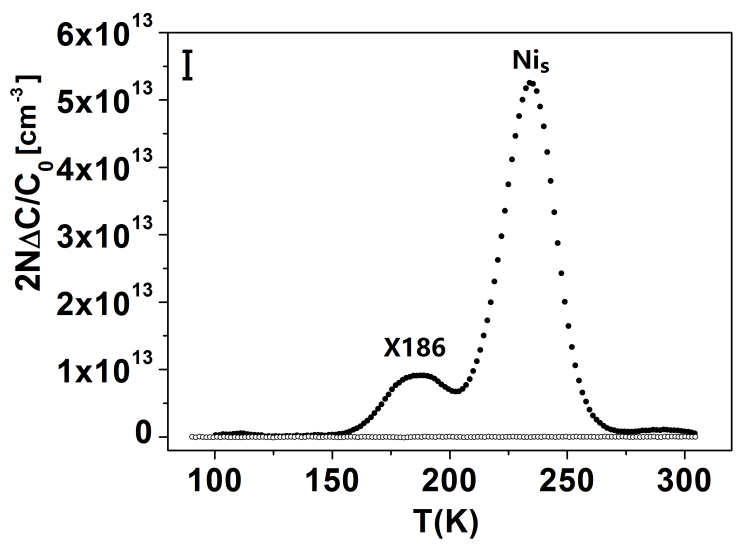

Figure 3.11: DLTS graphs of the as-grown state (open circles) and after nickel in-diffusion (filled circles), indicating electrically inactive complexes in the as-grown state, including weakly bound vacancies in concentrations to $C_{V, \max }=3 \times 10^{14} \mathrm{~cm}^{-3}$.

First indications for the conversion reactions are found after annealing at $500{ }^{\circ} \mathrm{C}$. The nitrogen level X186 occurs sporadically, by trend, with higher concentrations in large distances to the wafer surface.

As explained in sec. 3.1.1, the X186 level is linked with the X295 level related to the same complex. Sometimes, additional levels at $110 \mathrm{~K}$ and $150 \mathrm{~K}$ are observed. The first could be associated with vacancy oxygen complexes VO (with levels around $E_{c}-(0.15 \ldots 0.18) \mathrm{eV}$ reviewed by Pichler [54, p.476]). A further description of the X295 level is neglected here, since it belongs to the X186 level, according to sec. 3.1.1. The corresponding complex is designated as X186 in the following.

\section{Formation of X186 and possible intermediate states}

At $575{ }^{\circ} \mathrm{C}$ the nitrogen-related levels are distinctively formed, fig. 3.12. Maximum concentrations around $5 \cdot 10^{13} \mathrm{~cm}^{-3}$ have been found, which is about 5 percent of the overall concentration of nitrogen atoms (compare section 1.1.1). This is in accordance with earlier findings [93, 94, 95], stating an electrically 
active fraction of nitrogen of several percent.

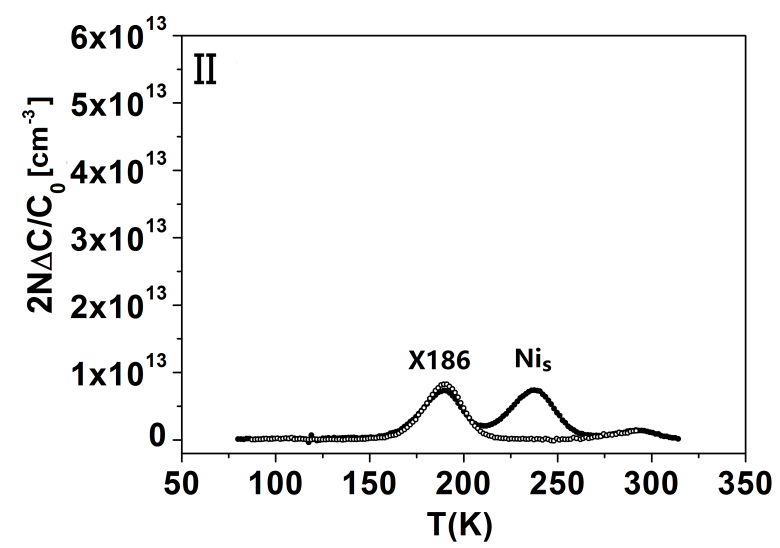

Figure 3.12: Conversion to the X186 complex with maximum concentrations of $C_{X 186}=6 \times 10^{13} \mathrm{~cm}^{-3}$ along with a loss of weakly bound vacancies.

The described findings show strong accordance with the observations by Grant et al. [25] of severe lifetime decrease after annealing, with minority carrier lifetimes partly below $100 \mu \mathrm{s}$. Applying the Shockley-Read-Hall recombination model to the midgap level the lifetimes can be calculated by

$$
\tau_{p}=\frac{1}{\sigma_{p}<v_{t h}>N_{T} \cdot f\left(N_{T}\right)}
$$

in n-type and

$$
\tau_{n}=\frac{1}{\sigma_{p}<v_{t h}>N_{T} \cdot\left(1-f\left(N_{T}\right)\right)}
$$

in p-type material. With the thermal velocity according to eq. 3.1, the occupation $f\left(N_{T}\right) \approx 1 / 3$, and by inserting the capture parameters, determined in section 3.1.1, we obtain minority carrier lifetimes of $\tau_{n}=285 \mu \mathrm{s}$ in p-type material and $\tau_{p}=5 \mu \mathrm{s}$ in n-type material. This provides evidence that the lifetime degradation in Grant et al. [25] is due to the near midgap level X295, related to the involvement of nitrogen and vacancies.

In the same temperature regime, where the nitrogen levels form, we observe that a substantial portion of vacancies gets lost for subsequent nickel decoration, implying that the vacancies are rearranged from their initial state. In order to trace back these vacancies in as-grown material, nickel in-diffusion was conducted close to the conversion temperature. As shown in the corresponding DLTS-graph in fig. 3.13, the trapping of nickel at the initial vacancies under 
these conditions is connected with a broadening of the X186 signal (filled dots). Once the native vacancies are rearranged, the broadening under nickel diffusion does not occur (empty dots). Assuming that the perturbations are caused by an overlap of the X186 defects with some $\mathrm{Ni}_{\mathrm{s}}$-induced electron states, one may speculate that a certain fraction of the nickel atoms, trapped at the initial vacancies is located in close proximity of the X186 complex. One may note that the hybridized electron states of the $\mathrm{Ni}_{\mathrm{s}}$ atoms can have a partly delocalized character, according to Haldane and Anderson [96]. No perturbations are observed at the acceptor level of the substitutional nickel here.
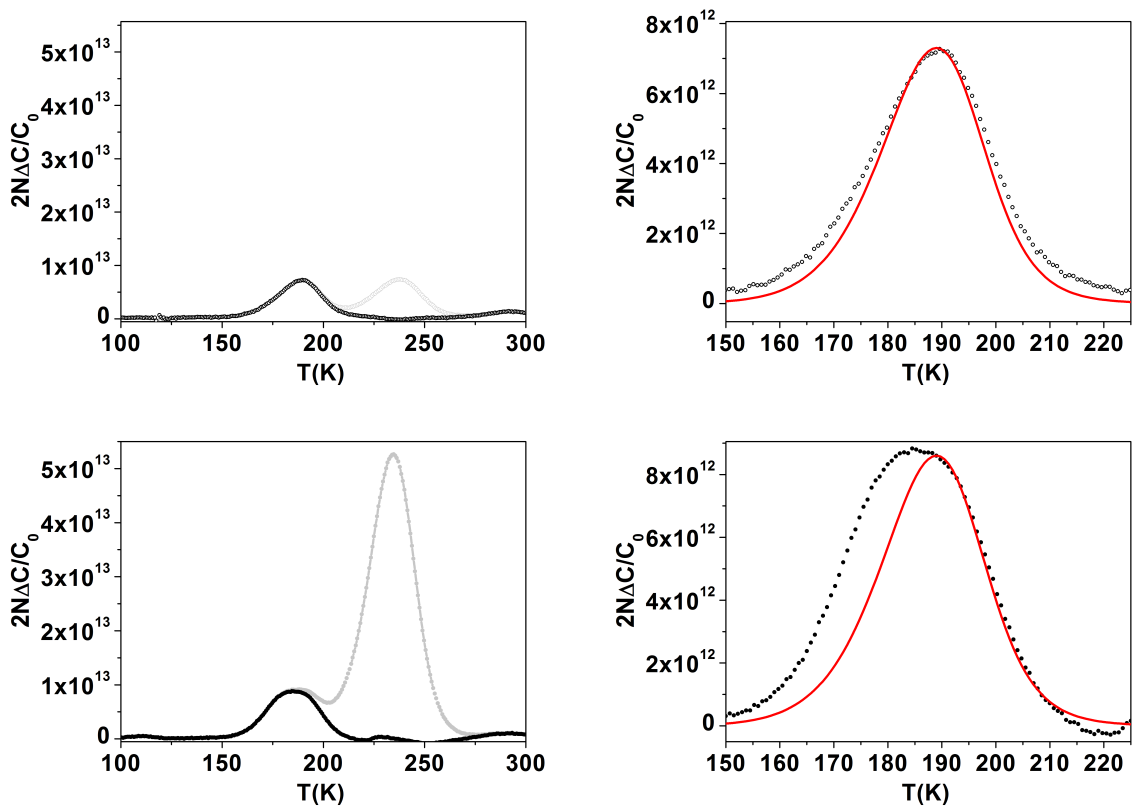

Figure 3.13: Analysis of the X186 DLTS-signature after nickel in-diffusion in FZsilicon after pre-annealing (top) and with native vacancy complexes (bottom). For both cases shown on the left: The X186 signature (black) was extracted by subtracting a synthetic nickel signature from the measured curves (grey). For both cases shown on the right: Comparison of the X186 signal (black dotted curve) with a synthetic X186 signal (red solid curve). After nickel in-diffusion in the presence of the native vacancy complexes (bottom right) the resulting X186-signal is perturbed.

The close vicinity of substitutional nickel to the X186 complex substantiates the idea that both, the initial vacancies and the X186 complex, originate from the same native complex. Its decomposition would then occur via multiple release of vacancies from the native state, so that the X186 complex remains. In the analyzed samples, the ratio of the detected concentrations between the X186 complex and initial weakly bound vacancies is roughly about 1:4 to 1:5. According to this stoichiometry, the prevailing native complexes could be the $\mathrm{NV}_{6}$ complex, decomposing in $\mathrm{NV}$ and $5 \mathrm{~V}$, or the $\mathrm{N}_{2} \mathrm{~V}_{10}$ complex, decomposing in $2 \mathrm{NV}$ and $8 \mathrm{~V}$. It is noticeable that the numbers of vacancies in these 
complexes match the first two magic numbers, associated with stable configurations of multivacancies, which are characterized by low numbers of dangling bonds [97]. The corresponding binding energies on the order of $2 \mathrm{eV}$ are smaller than the formation energies of $N_{s}$ [54]. This is qualitatively in accordance with the annealing behaviour at low temperatures observed in our experiments and substantiates the description of the native defects as a composition of a nitrogen part and a multivacancy part.

The proposed structure of the native complexes is in accordance with earlier observations of the nitrogen-controlled formation of voids below the delineation limit by Schmolke et al. [23]. In their explanation binding of vacancies to nitrogen slows the vacancy diffusion, resulting in slowed void nucleation. The current results given above imply that nitrogen complexes occasionally act as nucleation sites themselves for the aggregation of excess vacancies, stabilizing them during crystal cooling in high concentrations of nitrogen-bound multivacancies.

In this concept the vacancy concentration, detected by nickel in-diffusion provides a measure for the efficiency of the suppression of detrimental voids by nitrogen-controlled multivacancy formation. For their decomposition during subsequent annealing above $500{ }^{\circ} \mathrm{C}$ many paths and intermediate stages are conceivable. In this context, the lower conversion temperatures (starting at $450^{\circ} \mathrm{C}$ ) in Grant et al. [25] are plausible when assuming that their annealing in dry oxygen is related to the injection of interstitials which probably promotes the dissociation of the native vacancy complexes.

\section{Decomposition of X186}

Applying annealing temperatures above $575^{\circ} \mathrm{C}$ the concentration of the X186 complex is found to decrease by a thermally activated process with increasing temperature (see fig. 3.10), formally described by an activation enthalpy of $1.6 \mathrm{eV}$. The substitutional nickel concentration follows this decrease, which indicates a successive annealing of all detected complexes with increasing temperatures [90]. After annealing at sufficiently high temperatures no deep levels or weakly bound vacancies can be found at all, see fig. 3.14.

Comparing inert annealing with nickel in-diffusion, shown in the DLTS graphs in fig. 3.15 from annealing at $680^{\circ} \mathrm{C}$, reveals that the amplitude of the X186 complex is reduced by nickel in-diffusion. Regarding the X186 complex as substitutional nitrogen, it can be understood that nickel atoms promote its decomposition by grabbing its vacancies. Potential residues of the X186 complex annealing evade electrical detection. 


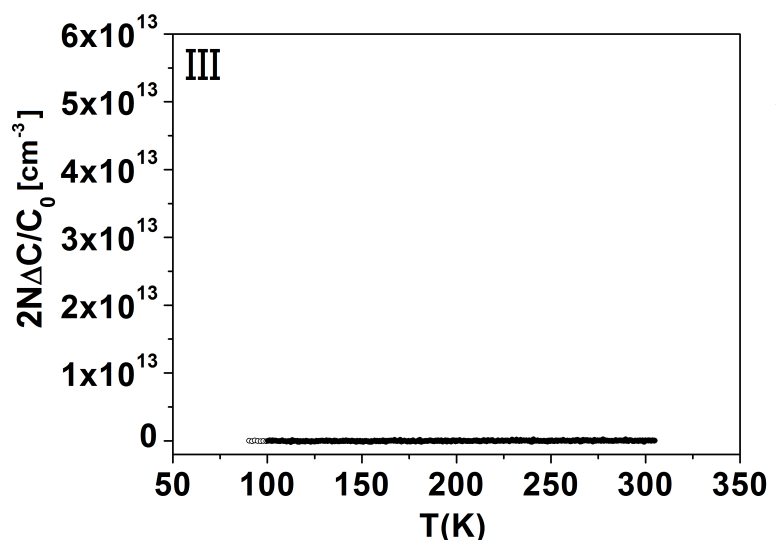

Figure 3.14: DLTS-graphs after annealing at $900^{\circ} \mathrm{C}$ with and without subsequent nickel in-diffusion shows no deep levels. This indicates an irreversible loss of the X186 complex and weakly bound vacancies with an annealing of all deep levels.

The underlying reactions are substantiated by energetic considerations:

- For substitutional nickel, First-Principles studies [98] predicted a binding energy of $E_{B}=2.7 \mathrm{eV}$ which is in accordance with the commonly accepted formation enthalpy of $\Delta H_{F, N i s}=3.1 \mathrm{eV}$ from solubility analyses, described in [6].

- For substitutional nitrogen, First-Principles calculations [99] estimated a binding energy of $E_{B, N s}=3.88 \mathrm{eV}$.

A favored vacancy binding to nickel becomes plausible when including the pairing of nitrogen atoms.

- Monomeric nitrogen atoms ought to pair to electrically inactive $N_{2}$ with binding energies, proposed earlier, ranging from $1.73 \mathrm{eV}$ via $3.67 \mathrm{eV}$, $3.86 \mathrm{eV}$ and $4.1 \mathrm{eV}$ to $4.3 \mathrm{eV}$ (Pichler [54], p.382).

The dissolution of the X186 complex, along with the preferred binding of vacancies to nickel atoms is then described by the reaction:

$$
\begin{aligned}
2 \mathrm{NV} & \longrightarrow \mathrm{N}_{2}+2 \mathrm{~V} \\
2 \mathrm{Ni} i_{i}+2 \mathrm{~V} & \longrightarrow 2 \mathrm{Ni} i_{s} .
\end{aligned}
$$

In the case of platinum marker in-diffusion [100], a stronger binding to vacancies, with binding energies for substitutional platinum, see e.g. in Jacob et al. [101], of $3.45 \mathrm{eV}$, would mean even larger driving forces for the decomposition 


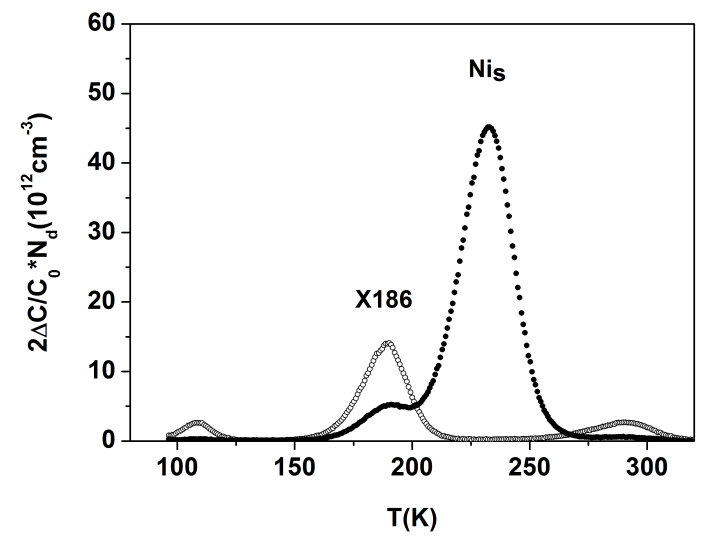

Figure 3.15: DLTS graphs after inert annealing (open circles) and nickel in-diffusion (filled circles) at $680^{\circ} \mathrm{C}$, showing a reduction of the X186 complex under nickel indiffusion. This indicates the decomposition of the X186 complex by vacancy release.

of the X186 complex. This would mean that the X186 complex is probably not to be seen in platinum in-diffusion experiments.

Once annealed out, the levels stayed absent during subsequent annealing at lower temperatures, which we carried out at some randomly selected samples. This indicates that their annealing reactions are irreversible. Possible loss mechanisms are figured out from the analysis of depth profiles of the nitrogen complex after time-dependent annealing, given in the appendix A.3, possibly with the involvement of additional complexes.

\section{Summary}

From the close proximity of the detected vacancies to the nitrogen complex, native complexes are proposed to consist of nitrogen and multivacancies, revealing stochiometries of $\mathrm{NV}_{6}$ or $\mathrm{N}_{2} \mathrm{~V}_{10}$, which have formed during crystal growth. Upon subsequent wafer-based annealing at temperatures between $T \approx 500{ }^{\circ} \mathrm{C}$ and $T \approx 700^{\circ} \mathrm{C}$ they are found to irreversibly decompose by vacancy release and residuing substitutional nitrogen. With increasing temperatures, all nitrogen-related deep levels and weakly bound vacancy complexes anneal out irreversibly, possibly associated with out-diffusion to the wafer surfaces.

\subsubsection{Additional dopants and structural defects: Alternative va- cancy reaction paths}

To examine the occurrence of weakly bound vacancies in crystalline silicon from other growth modes, the nickel marker in-diffusion procedure was carried 
out at standard Czochralski silicon and block cast multicrystalline and quasi monocrystalline silicon. The corresponding DLTS graphs, placed in the appendix A.4, revealed no hints for nitrogen vacancy complexes or weakly bound vacancies.

Their absence can be explained by considering that in these materials, additional agents as oxygen or structural defects are present in higher concentrations and provide alternative reaction paths during crystal cooling by consuming vacancies and forming more stable complexes. Upon the growth of $\mathrm{Cz}-$ silicon, vacancies are supposed to enter the complexing with oxygen, present in a concentration two orders higher than in FZ-silicon, and the formation of the energetically more stable $\mathrm{VO}_{2}$-complex is supposed to prevail [20].

In multicrystalline silicon, vacancies are assumed to equilibrate at dislocations. Simulations of the dislocation-assisted Frenkel recombination in a typical cooling process of block-cast crystallization with a dislocation density of $N_{\text {disl }}=10^{4} \mathrm{~cm}^{-2}$ are shown in fig. 3.16 for assumed cooling rates of $d T / d t=1000 \mathrm{~K} / \mathrm{h}(\mathrm{a})$ and $d T / d t=100 \mathrm{~K} / \mathrm{h}(\mathrm{b})$. For both cooling rates can be seen that the point defect concentrations (solid curves) instantaneously follow their equilibrium concentrations (dashed curves), given in the appendix A.1.3. Further vacancy reactions, related to dislocation kinetics have recently been discussed by Kveder et al. [102] and Khorosheva et al. [103].

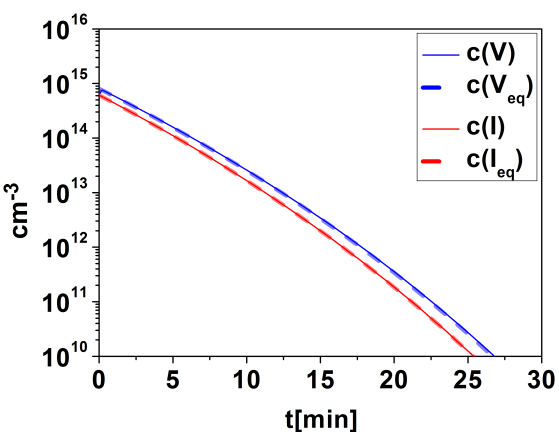

(a)

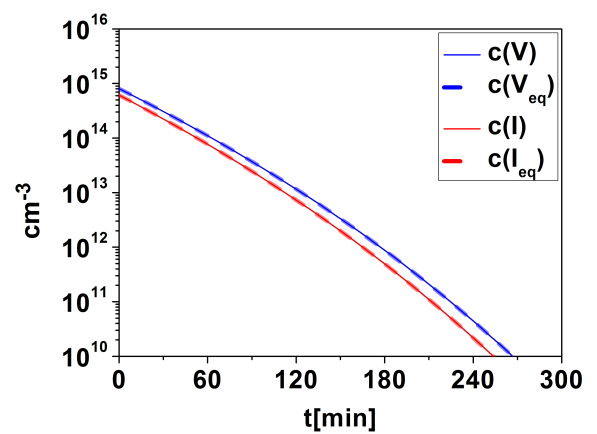

(b)

Figure 3.16: Simulated curves for the intrinsic point defect concentrations during the solar silicon block cast process under standard conditions with cooling rates (a) of $1000 \mathrm{~K} / \mathrm{h}$ and $(\mathrm{b})$ of $100 \mathrm{~K} / \mathrm{h}$, modeling Frenkel pair recombination at dislocations in densities of $10^{4} \mathrm{~cm}^{-2}$. In both cases during all cooling stages, the intrinsic point defect concentrations (of interstitials: red line, of vacancies: blue line) are close to their equilibrium concentrations (respective dashed lines) which identifies the intrinsic point defect annihilation at dislocation as a strong reaction path that ought to be predominant here. 


\section{Summary}

In other silicon materials with additional dopants and structural defects, the absence of weakly bound vacancies indicates that alternative vacancy reaction paths prevail during crystal growth, including reactions to stable complexes or interactions with dislocations. 


\subsubsection{Advantages and drawbacks of the nickel marker in-diffusion technique}

The applicability of the vacancy marker method depends in the end on the substitution of the initial vacancy binding partners by the marker atoms. On the one hand, the binding energies must be large enough to ensure a favourite binding of vacancies to the marker under the given marker concentrations. On the other hand, the binding energies must not be too large to ensure that the marker does not replace the silicon lattice atoms by the kick-out mechanism.

In this context, substitutional nickel distinguishes itself from earlier marker species by having rather small binding energies. The implementation of nickel marker diffusion within the analysis of grown-in complexes in FZ-silicon has been proven powerful to selectively probe weakly bound vacancies in successive annealing stages. Comparably low formation energies of interstitial nickel facilitated large nickel diffusion fluxes under little invasive conditions to approach the native vacancy complexes apart from parasitic reactions as premature annealing or kick-out reactions. The control of nickel-related side reactions ensured the exclusive formation of substitutional nickel.

According to our findings, more stable complexes, such as $\mathrm{VO}_{2}$ or possible posterior annealing stages, evade the detection with nickel. Therefore, the nickel marker diffusion method, compared to Pt-diffusion and Au-diffusion, is sensitive to weakly bound vacancies. Thus, the combination of nickel marker in-diffusion and gold marker in-diffusion facilitates the approach of vacancy binding energies, as was performed for vacancy trail defects after dislocation moving by Kveder et al. [102].

The analysis showed that typical native vacancy concentrations in FZ-silicon can have a decisive effect on the kinetics of additional dopants and their concentrations established during subsequent processes. With respect to silicon processing, deliberate nickel in-diffusion into vacancy-rich FZ-silicon at medium temperatures could establish high excess concentrations of substitutional nickel, which are comparable to equilibrium concentrations around $1200^{\circ} \mathrm{C}$ - at much lower process temperatures. The achievable doping concentrations of such hypothermal doping can be tuned by the adjustment of previous vacancy complexes under pre-annealing at medium temperatures.

\section{Summary}

Nickel marker diffusion has been proven to be a powerful technique in the analysis of vacancy-related grown-in complexes in FZ-silicon. The low formation energies eventually enable large in-diffusion fluxes under little invasive processes. Due to the comparably low binding energies of nickel to vacancies, 
stable voids or $\mathrm{VO}_{2}$ evade their detection with nickel. Therefore, nickel is a selective marker for weakly bound vacancies. 


\subsection{Correlation between vacancy concentration and nickel disilicide nucleation}

In many gettering and defect engineering processes, the kinetics of precipitates play a key role [1]. An understanding of their formation is crucial. Recent simulations covering precipitation kinetics [27, 104, 105], require predictions about their initial stages. Therefore, a better understanding of early nucleation stages on atomic scale is essential. Experiments on this subject

are sparse. Early stages of copper precipitation have been examined in DLTS analyses [28] and First-Principles studies [29], stating agglomerates with four atoms of three interstitial and one substitutional copper atoms. The current study aims to experimentally access the early nucleation stages of nickel disilicide precipitation, i.e. the stages prior to the introduction of the bounding dislocation and the related stacking fault.

Structural analyses of highly coherent macroscopic $\{111\}$-interfaces by Cherns et al. [106] and precipitates by Seibt and Schröter [9] showed that the positions of the nickel atoms in the $\mathrm{NiSi}_{2}$ phase correspond to substitutional sites of the adjacent silicon lattice. This raises the question, if substitutional nickel is involved already in the nucleation process. From section 3.1 it is known that the substitutional nickel concentration is linked with the concentration of pre-existing vacancy complexes that can be controlled in a pre-annealing procedure. In section 3.2.1, the effect of substitutional nickel, formed with pre-existing vacancies, on the nickel disilicide precipitation is analyzed. The resulting precipitate densities as a measure for the nucleation efficiency are determined by DLTS, preferential etching and EBIC: DLTS-analysis additionally reveals the corresponding concentrations of substitutional nickel, Preferential etching analysis reveals to directly access the precipitate densities, EBIC analysis reveals a link to the corresponding recombination activity. Complementary, in section 3.2.2, the effect of precipitation on the substitutional nickel concentration is investigated by DLTS. In section 3.2.3 possible nucleation paths, including early stages without and with substitutional nickel, are proposed.

\subsubsection{Substitutional nickel increases precipitation}

The formation of precipitates was analyzed in two principal setups: with and without the presence of substitutional nickel. For this purpose, precipitates were generated upon nickel diffusion in a sample from initial state FZ-material providing weakly bound vacancies as nickel traps and, in the same run, in a sample in which the nitrogen-vacancy-complexes had been eliminated by preannealing. The in-diffusion of nickel was immediately finished by quenching in sodium hydroxide. The procedure is sketched in the block diagram in fig. 3.17 . 


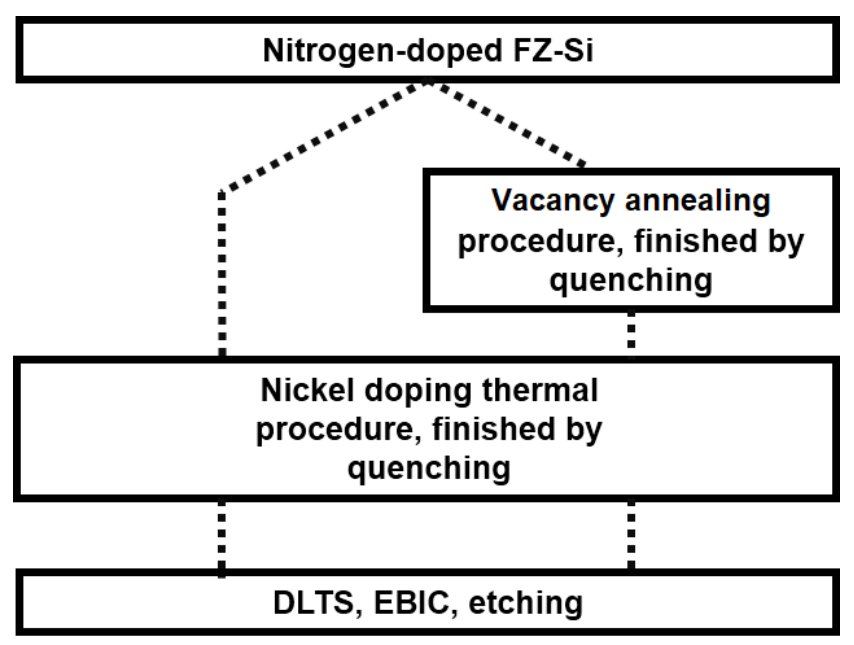

Figure 3.17: Block diagram, showing the preparation process for nickel disilicide precipitation with and without vacancy annealing.

\section{DLTS analysis}

Typical DLTS-graphs after nickel precipitation are shown in fig. 3.18. Without vacancy annealing, the high temperature side of the precipitate signature (dashed curves in fig. 3.18(a)) is superimposed by the nickel acceptor point defect line. To obtain the pure precipitate signature the substitutional nickel signal was subtracted by simultaneous least squares fitting the high temperature sides of different capture pulse durations to their mean value by respectively subtracting a synthetic point defect line (dash-dotted curves) according to the acceptor state of substitutional nickel with $\sigma=6 \times 10^{-17} \mathrm{~cm}^{2}$ and $\Delta H=0.43 \mathrm{eV}$. The resulting curves (solid line) comply very well with the shape of the pure bandlike DLTS signature of the precipitates, decoupled from the substitutional nickel point defect line.

After vacancy annealing, shown in the viewgraph 3.18 (b), the spectra show the pure bandlike DLTS signature and no point defect line is detected. The amplitude of the signal is smaller by approximately a factor of 5 .

Comparing the spectra of both cases for the same pulse duration $t_{p}=10 \mu \mathrm{s}$, fig. 3.19, reveals significant differences in the width of the spectra. According to the evaluation by Riedel [10], introduced in section 1.3.1, different precipitate sizes can be deduced. From the precipitate growth after vacancy annealing, the full width half maximum of the precipitate signal is $\Delta T_{F W H M}=50 \mathrm{~K}$ implying precipitate diameters around $39 \mathrm{~nm}$. Considering that the interstitial nickel at the in-diffusion temperature is completely consumed by the precipitates [9] one can deduce a volume density of the precipitates, revealing $N_{\text {prec }}=4 \times 10^{12} \mathrm{~cm}^{-3}$ which corresponds very well to the results of Riedel after precipitation at $900^{\circ} \mathrm{C}$. 


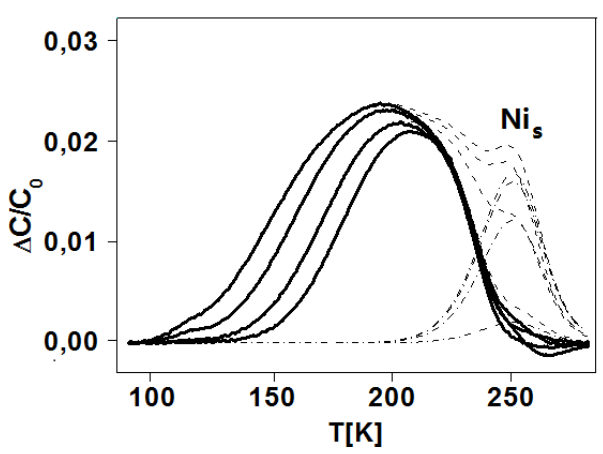

(a)

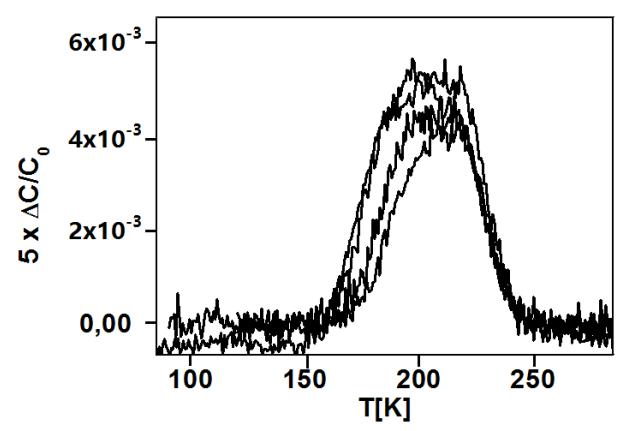

(b)

Figure 3.18: DLTS signal of nickel disilicide precipitates from nickel in-diffusion in as-grown samples in which substitutional nickel formed preceding the precipitation process (a) and of precipitates from nickel in-diffusion after vacancy annealing (b). Under the effect of the substitutional nickel (a), the signal is broadened to the lowtemperature side and the high temperature side is superimposed by the acceptor point defect line of substitutional nickel. After subtraction of a synthetic substitutional nickel signature (dash-dotted curves), one obtains the isolated precipitate spectrum (solid curves). The scale of the ordinate differs between both plots by approximately a factor of 5 . The spectra are taken with a correlation frequency of $f=68 \mathrm{~Hz}$ and capture pulse durations of $t_{p}=10^{-7} \mathrm{~s}, 10^{-6} \mathrm{~s}, 10^{-5} \mathrm{~s}$ and $10^{-4} \mathrm{~s}$.

From the precipitate growth without vacancy annealing, the full width half maximum of the precipitate signal is $\Delta T_{F W H M}=85 \mathrm{~K}$ implying precipitate diameters around $12 \mathrm{~nm}$, corresponding to a precipitate density of $4 \times 10^{13} \mathrm{~cm}^{-3}$. This indicates that the presence of substitutional nickel during precipitation results in significantly increased precipitate densities indicating a higher nucleation rate.

\section{Preferential etching analysis}

The results are corroborated by the analysis of the precipitate distribution in defect preferential etching experiments performed at the same parallel samples. The figures 3.20 and 3.21 display the revealed precipitate patterns. Both samples had been etched under identical conditions in different runs with Secco etching solution with the composition, given in [107]. The etching rate of $0.6 \mu \mathrm{m} / \mathrm{min}$ (see Gnauert [69], p.40) and the etching time 10s correspond to a projected depth of $100 \mathrm{~nm}$. The etch pitchs have been counted numerically by identifying and counting connected areas below a grey level threshold. In the case after vacancy annealing, the maps show a homogenous distribution of etch pits pursuant to a precipitate density of $N_{\text {prec }}=8 \times 10^{12} \mathrm{~cm}^{-3}$. In the case without vacancy annealing, the overview image shows alternately arranged bright and dark streaks with a lateral distance of about $50 \mu \mathrm{m}$, possibly 


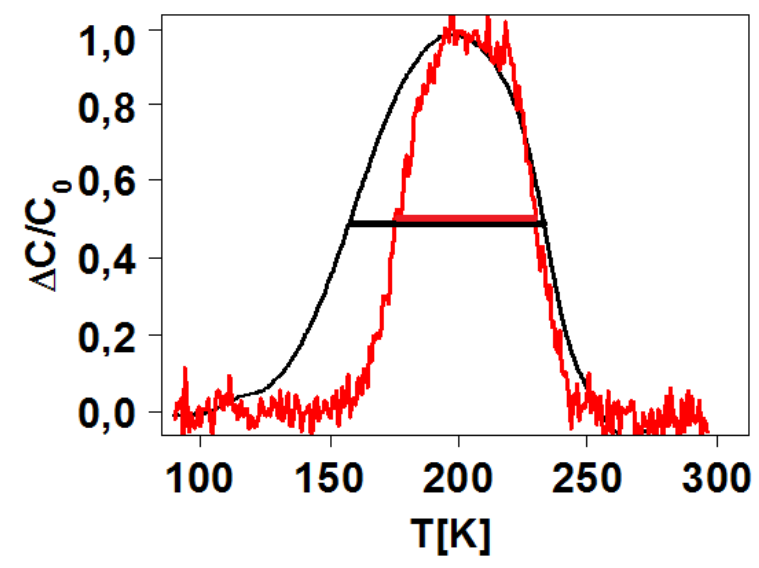

Figure 3.19: Comparison of the DLTS signals (normalized to their respective maximum) after precipitation with and without substitutional nickel. The larger FWHM in the presence of substitutional nickel indicates a larger density of smaller precipitates.

due to inhomogeneous distributions of the pre-existing defects. An effect of striations on the $\mathrm{Cu}_{3} \mathrm{Si}$ precipitation has been observed by Graff [108]. Similar inhomogeneities have been observed in EBIC analyses but are not further discussed here. The corresponding precipitate densities in the bright region and in the dark region are $8 \times 10^{13} \mathrm{~cm}^{-3}$. In accordance with the DLTS-results, the preferential etching experiments indicate an increased precipitate density without vacancy annealing by roughly a factor of 10 . Between the precipitate densities of both techniques lies a systematic factor of two, which is within the error regime assumed to be caused by uncertainties in the etching procedure. 


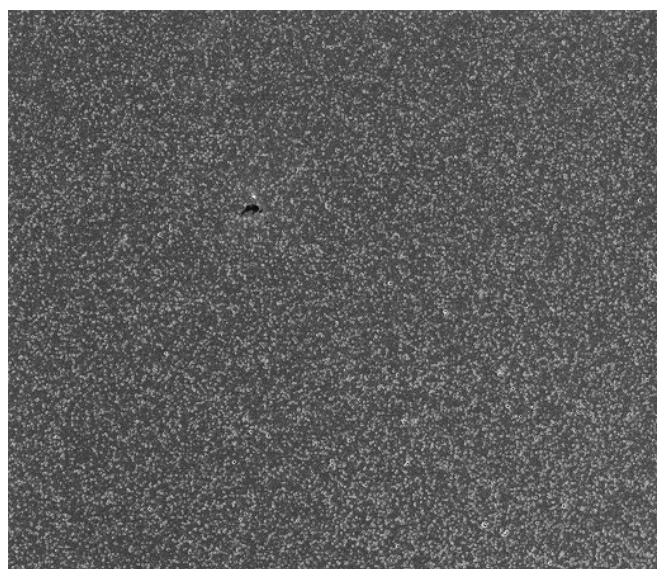

$50 \mu \mathrm{m}$

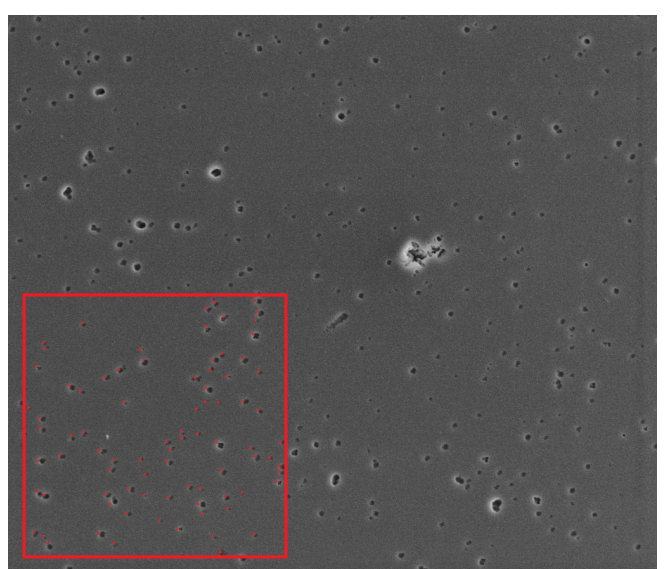

$5 \mu \mathrm{m}$

(a)

(b)

Figure 3.20: SEM micrographs of the precipitate distribution after precipitation without substitutional nickel, visualized by 10s Secco etching, corresponding to a projected depth of $100 \mathrm{~nm}$. The overview (a) supports the assumption of a homogeneous precipitate distribution. The red square in the magnified section (b) with an edge length of $10 \mu \mathrm{m}$ contains 84 etch pits, corresponding to a precipitate density of $N_{\text {prec }}=8.4 \times 10^{12} \mathrm{~cm}^{-3}$.

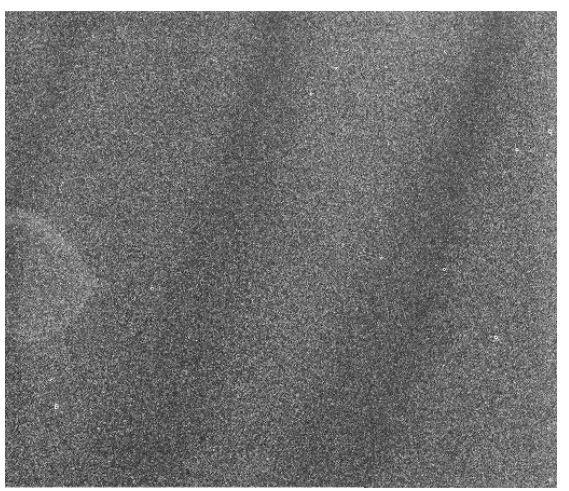

$50 \mu \mathrm{m}$

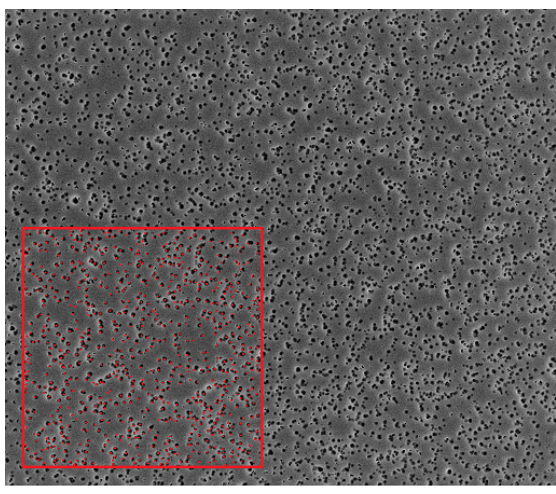

$5 \mu \mathrm{m}$

(a)

(b)

Figure 3.21: SEM micrographs of the precipitate distribution after precipitation with substitutional nickel, visualized by 10s Secco etching, corresponding to a projected depth of 100nm. The overview (a) supports the assumption of a homogeneous precipitate distribution. The red square in the magnified section (b) with an edge length of $10 \mu \mathrm{m}$ contains 754 etch pits, corresponding to a precipitate density of $N_{\text {prec }}=7.9 \times 10^{13} \mathrm{~cm}^{-3}$. 


\section{EBIC analysis}

EBIC micrographs after precipitation without and with substitutional nickel are shown in fig. 3.22. The explicit determination of the precipitate densities is hampered by the overlapping of the individual contrast features. A qualitative analysis shows an increased recombination activity after precipitation without vacancy annealing, with a shift of the maximum in the histograms to lower currents. Increasing recombination activity with increasing precipitate concentration under equal overall concentration of precipitated metal is in accordance with the theoretical studies by Plekhanov and Tan [109] and with the results by Kittler et al. [75] for $\mathrm{NiSi}_{2}$ precipitates after ripening.
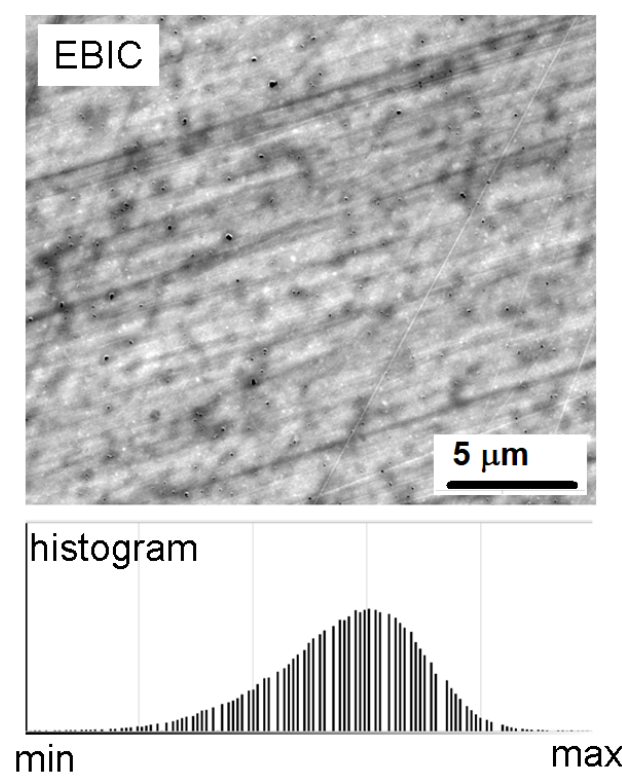

(a)
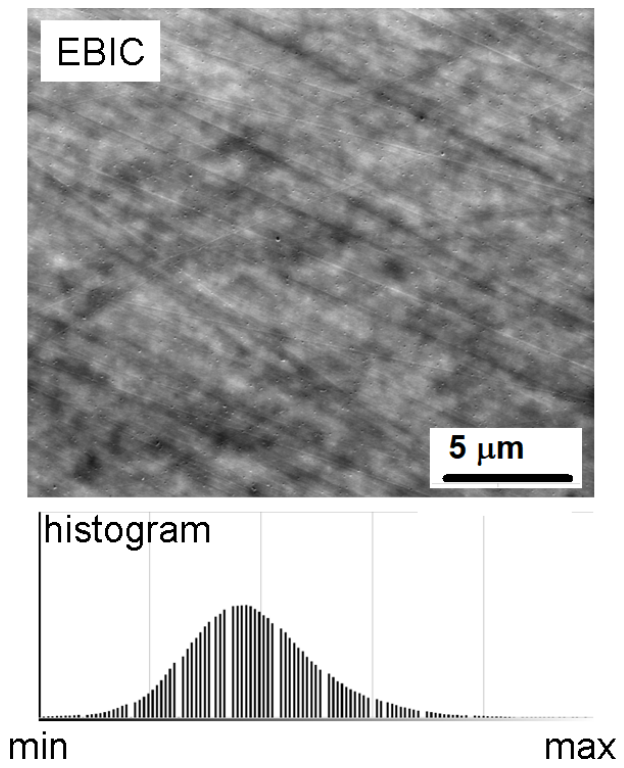

(b)

Figure 3.22: EBIC-micrographs of precipitates without (a) and with (b) available substitutional nickel. The resolution of single precipitates is hampered by an overlapping of the individual signatures. The histograms below indicate a larger region with reduced signal after precipitation with available substitutional nickel.

\section{Temperature dependence}

The precipitate densities after quenching in $\mathrm{NaOH}$ from different annealing temperatures are summarized in fig. 3.23. The black region comprehends the precipitate densities after quenching without $\mathrm{Ni}_{\mathrm{s}}$. In the case with $\mathrm{Ni}_{\mathrm{s}}$, the precipitate densities are comprehended in the blue region. The increased precipitate density in the presence of substitutional nickel, indicated through 
all temperatures, provides strong indications that substitutional nickel atoms lower the nucleation barrier for nickel disilicide precipitation.

In addition, earlier data by Riedel [10] are included in fig. 3.23 by the red region. In that study, the spectra after nickel in-diffusion and precipitation have revealed an additional DLTS signal at 240K, whose origin had not clearly been understood. It remained e.g. unexplained, why the concentration of the corresponding level was higher for lower annealing temperatures, being most apparent after annealing temperatures below $850^{\circ} \mathrm{C}$. Against the background of the current results, presented in section 3.1.2, it seems very likely that the additional point defect is substitutional nickel, formed by trapping of interstitial nickel during the annealing process at the initial weakly bound vacancies. In full accordance with this explanation, the precipitate densities in Riedel's study are located in the blue region at the low temperature side. On the high temperature side, the data by Riedel coincide with the case without $\mathrm{Ni}_{\mathrm{s}}$. The current precipitate concentrations are two to three orders of magnitude larger than the density of precipitates, grown under smaller quenching rates with ethylene glycol by Seibt [63]. For the temperature region and the annealing times applied there, $\mathrm{Ni}_{\mathrm{s}}$ most probably had annealed.

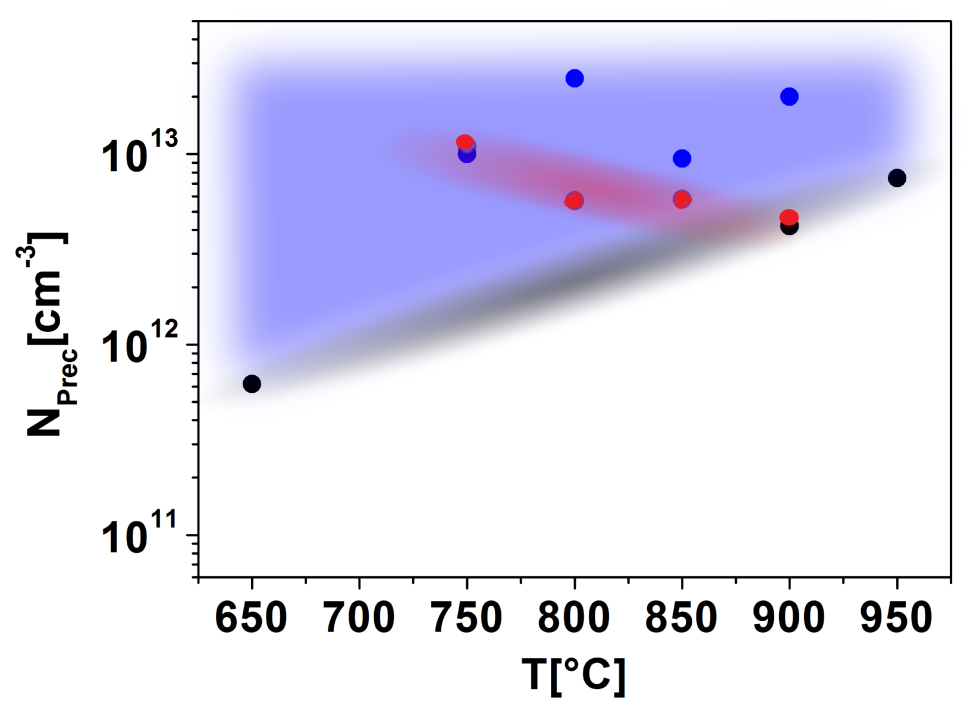

Figure 3.23: Temperature-dependent precipitate densities from DLTS-data without substitutional nickel (black circles)and with substitutional nickel (blue circles), compared to the data by Riedel [10] (red circles). The datapoints are underlayed respectively to their colours, by which the different regimes are separated.

From the precipitate densities, summarized in table 3.2, and the concentration of potential nucleation sites, for the case without substitutional nickel (given by the concentration of silicon lattice sites $N_{S i}=5 \times 10^{22} \mathrm{~cm}^{-3}$ ) and with substitutional nickel $\left([N i]_{s}\right)$, one can derive that the nucleation probability 
is increased by a factor of about $10^{9}$ for nucleation at a substitutional nickel atom. According to eq. 1.8 in section 1.2 .3 for a temperature of $T=900^{\circ} \mathrm{C}$ this corresponds to a difference in the nucleation barriers of $1.7 \mathrm{eV}$ and gives strong evidence for the involvement of substitutional nickel in the early nucleation stages. In the case without pre-existing substitutional nickel, the formation of substitutional nickel may thus be the limiting process for $\mathrm{NiSi}_{2}$ nucleation or alternative nucleation paths exist.

\section{Summary}

DLTS, preferential etching and EBIC analyses revealed evidence for efficient nucleation when high concentrations of substitutional nickel are present, formed at pre-existing vacancies. The probability for $\mathrm{NiSi}_{2}$ nucleation at a substitutional nickel site is increased by a factor of about $10^{9}$ corresponding to a decrease of the nucleation barrier of $1.7 \mathrm{eV}$. 


\subsubsection{Decrease of substitutional nickel concentration under precipitation}

What happens with the substitutional nickel during precipitation? In a crosscheck experiment, the effect of precipitation on the substitutional nickel concentration was examined. For this purpose, substitutional nickel was formed at weakly bound vacancies by nickel in-diffusion in sample pairs of FZ-material without vacancy annealing. The annealing was finished for one of the samples by quenching to promote precipitates, and for the other sample by moderately fast cooling with a cooling rate of $-4 \mathrm{~K} / \mathrm{s}$ to $-6 \mathrm{~K} / \mathrm{s}$ precipitate the interstitial nickel at the surface, according to [110], and to freeze the substitutional nickel concentration without precipitation in the bulk. The preparation is illustrated in fig. 3.24.

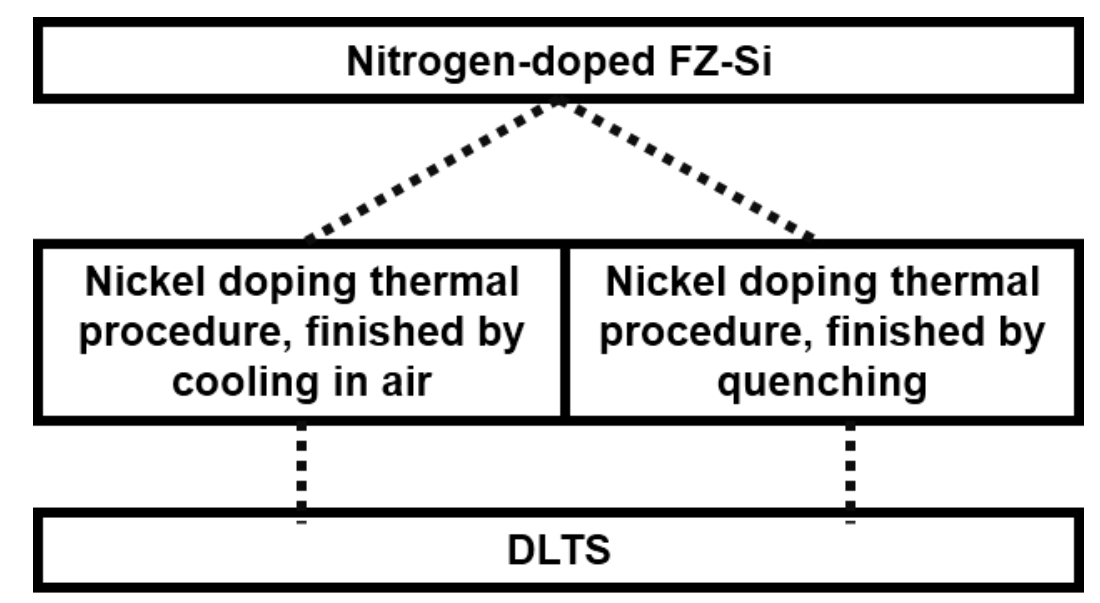

Figure 3.24: Block diagram of the preparation process for formation of substitutional nickel with and without subsequent quenching.

DLTS-profiles, illustrated in fig. 3.25 show that the detected substitutional nickel concentration is decreased after precipitation according to a consumption of substitutional nickel atoms. For different in-diffusion temperatures, the decrease in concentration of substitutional nickel is shown in fig. 3.26 (blue bars) in comparison with the corresponding precipitate densities (black bars).

For each temperature, the decrease in substitutional nickel concentration is even larger than the detected precipitate densities. This reveals indications for the formation of some invisible clusters. This may provide indications for $\mathrm{NiSi}_{2}$-stages, which did not undergo a transformation to a stacking fault. It is not clear if they remain in the material as invisible clusters or if they completely dissolve in favour of the transformed precipitates, as a result of Ostwald ripening (generally described in Haasen [111]). From different experimental conditions, Lemke [112] postulated the formation of $\mathrm{Ni}_{\mathrm{s}} \mathrm{Ni}_{\mathrm{i}}$-pairs. These complexes have been described to form during growth of a doped $\mathrm{Ni}$ crystal in certain atmosphere. They were found to be electrically inactive and 


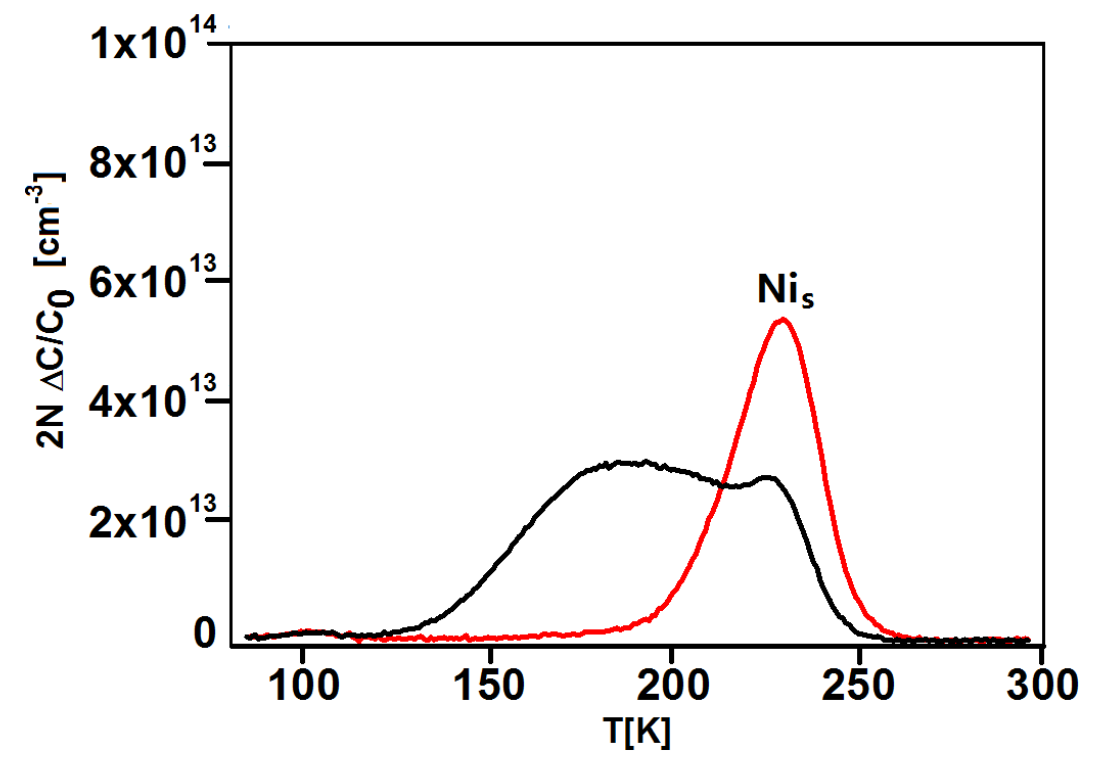

Figure 3.25: DLTS-graphs after nickel in-diffusion in FZ-samples without pretreatment, finished by moderate cooling (red) and by precipitation (black). Under precipitation, the concentration of substitutional nickel is decreased.

dissociated in a subsequent anneal at $600{ }^{\circ} \mathrm{C}$. Further properties of the pair are not known yet. It seems possible to us that these invisible pairs also occur in our experiment contributing to the losses of the observed substitutional nickel concentration.

The data are summarized in table 3.2. The corresponding spectra are given in the appendix A.5.

\section{Summary}

DLTS analyses indicate that the decrease in substitutional nickel concentration is slightly higher than the resulting precipitate densities, suggesting that more substitutional nickel is consumed than precipitates are finally formed. Probably, some invisible clusters have formed that do not transform to stacking faults or even completely dissolve in favour of the transformed precipitates. 


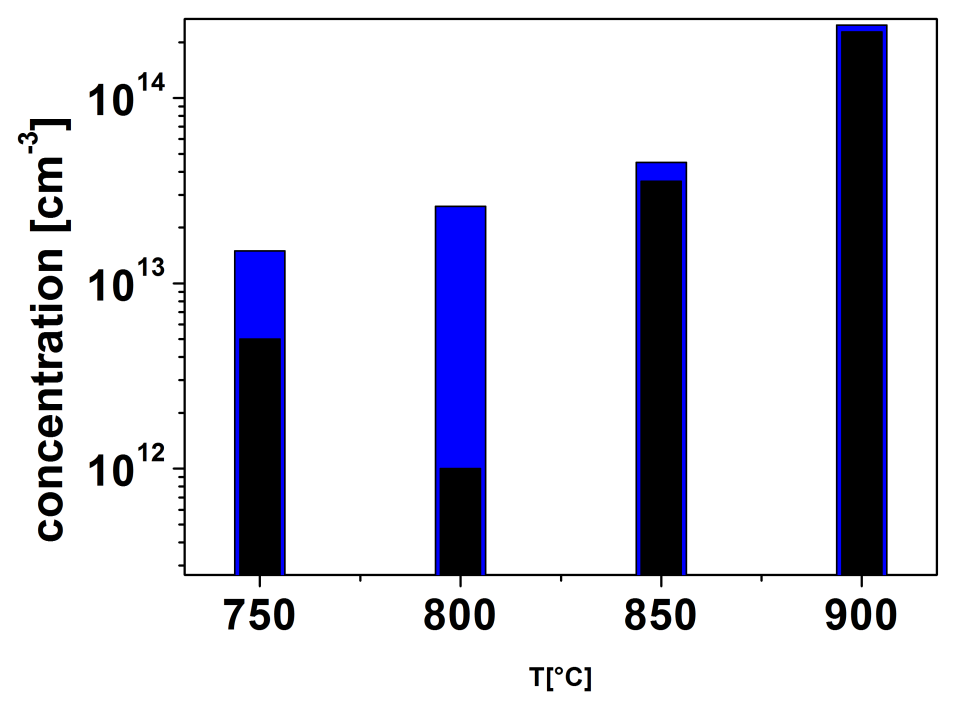

Figure 3.26: Decrease of substitutional nickel under precipitation (blue bars) and precipitate density (black bars). The difference between them indicates the formation of invisible $\mathrm{NiSi}_{2}$-agglomerates that do not undergo a transformation to a stacking fault.

\begin{tabular}{|c|c|c|c|c|c|}
\hline$T /{ }^{\circ} \mathrm{C}$ & $\begin{array}{l}c\left(N i_{s}\right) / \mathrm{cm}^{-3} \\
\text { without prec }\end{array}$ & $\begin{array}{c}c\left(N i_{s}\right) / \mathrm{cm}^{-3} \\
\text { with prec }\end{array}$ & FWHM & $\mathrm{d}$ & $N_{\text {prec }} / \mathrm{cm}^{-3}$ \\
\hline 650 & 0 & 0 & 83 & $10( \pm 5)$ & $6.2 \times 10^{11}$ \\
\hline 750 & $1.1 \times 10^{14}$ & $9.5 \times 10^{13}$ & 120 & $\leq 7$ & $\geq 1 \times 10^{13}$ \\
\hline $750^{*}$ & no data* & no data* & $93^{*}$ & $7( \pm 3)^{*}$ & $1.1 \times 10^{13 *}$ \\
\hline 800 & $7.9 \times 10^{13}$ & $5.3 \times 10^{13}$ & 106 & $\leq 7$ & $\geq 2.5 \times 10^{13}$ \\
\hline $800^{*}$ & no data* & no data* & $75^{*}$ & $15 \overline{( \pm 7)^{*}}$ & $\overline{5} .7 \times 10^{12 *}$ \\
\hline 850 & $6 \times 10^{13}$ & $1.5 \times 10^{13}$ & 71 & 17 & $9.5 \times 10^{12}$ \\
\hline $850^{*}$ & no data* & no data* & $58^{*}$ & $22( \pm 4)^{*}$ & $5.8 \times 10^{12 *}$ \\
\hline 900 & $3 \times 10^{14}$ & $5.3 \times 10^{13}$ & 71 & $17( \pm 7)$ & $2.0 \times 10^{13}$ \\
\hline $900 *$ & no data* & $0^{*}$ & $56^{*}$ & $37( \pm 6)^{*}$ & $4.2 \times 10^{12 *}$ \\
\hline 900 & 0 & 0 & 55 & $37( \pm 6)$ & $4.2 \times 10^{12}$ \\
\hline 950 & 0 & 0 & 50 & $39( \pm 6)$ & $7.5 \times 10^{12}$ \\
\hline
\end{tabular}

Table 3.2: Diffusion temperature (T), pre-existing substitutional nickel concentration $\left(N i_{s}\right)$, substitutional nickel concentration after precipitation, the full width half maximum of the precipitate peak (FWHM), corresponding precipitate diameter $\mathrm{d}$ and the deduced precipitate density $N_{\text {prec }}$. Previous data by Gnauert [69] and Riedel [10] are marked by *. 


\subsubsection{Possible early $\mathrm{NiSi}_{2}$ nucleation stages}

Proving the ability of substitutional nickel atoms as preferred nucleation sites, the in-situ experimental detection of the highly transient stages in the early nucleation process on atomic scale is practically impossible yet. The aim in this part is to propose a model of possible configurations of early $\mathrm{NiSi}_{2}$ nucleation stages. For this purpose, the nucleation process is assumed as consecutive agglomeration of nickel atoms to the growing nuclei. The approach considers atomic configurations along nucleation paths referring to initial stages with and without substitutional nickel.

\section{Atomic structure}

The coherence of the $\mathrm{NiSi}_{2}$ phase with the host lattice [9] provides strong reasons to consider that the nucleus has $\mathrm{NiSi}_{2}$ structure. The smallest imaginable $\mathrm{NiSi}_{2}$ unit consists of a nickel atom on a substitutional site of the host lattice between a silicon atom on a neighbored host lattice site and a silicon atom on one of the four tetrahedral interstitial sites ('antibonding sites'). The structure is shown in fig. 3.27. It represents a basis of the $\mathrm{NiSi}_{2}$-phase in the cubic fcc bravais lattice.

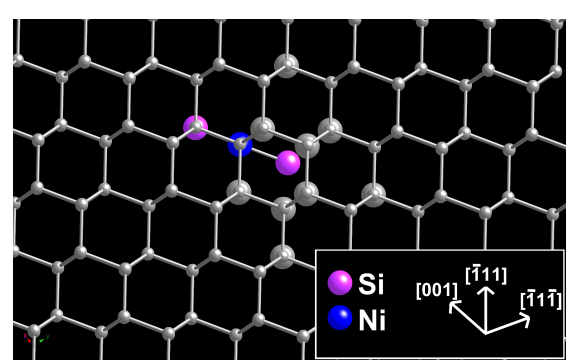

Figure 3.27: Smallest unit of $\mathrm{NiSi}_{2}$ precipitate, consisting of two silicon atoms (pink) and one nickel atom (blue). The grey atoms represent the host lattice. The enlarged atoms represent one fcc-unit cell.

In the case without substitutional nickel, the basic unit can be formed, when an interstitial nickel atom occupies a substitutional site by replacing the silicon atom, formerly on that site, to a tetrahedral interstitial site. The mechanism is illustrated in fig. 3.28 in the left column 'without substitutional nickel' from I to II. In sum, by consumption of an interstitial nickel atom, one $\mathrm{NiSi}_{2}$-unit is formed.

In the case with substitutional nickel already present, the basic unit can form at the existing substitutional nickel atom, when it attracts a silicon atom from a next nearest lattice site, providing a trap for a further nickel atom. The mechanism is illustrated in fig. 3.28 in the right column 'with substitutional 
nickel' from I to II. In sum, by consumption of an interstitial nickel atom, one basic unit and an additional Ni-Si-bond, in the following designated as 'Ni-Si-interbond' is formed.

\section{Formation enthalpies}

To estimate the formation enthalpy for the formation of a basic $\mathrm{NiSi}_{2}$-unit, we consider its formation to be composed of the following parts:

- Formation of a vacancy and a self interstitial: The formation enthalpies of a vacancy and a self interstitial have been considered as the sum of the single formation enthalpies from computed values by Sinno et al. [113] fitted to data from crystal growth analyses, revealing $\Delta H=6 \mathrm{eV}$.

- Formation of a substitutional nickel atom from an interstitial nickel atom and a vacancy: The energy for the formation of a substitutional nickel atom from a vacancy and an interstitial nickel atom has been calculated in first principles studies $(T=0 \mathrm{~K})$ by Backlund and Estreicher [58], revealing $\Delta H=-2.7 \mathrm{eV}$.

- Formation of a Ni-Si-interbond: The energy of a Ni-Si bond is estimated from the energy of the formation of substitutional nickel $2.7 \mathrm{eV}$ divided by 4 , revealing $\Delta H=-0.68 \mathrm{eV}$. Alternatively, the formation of a Ni-Si-bond is considered with the elimination of a silicon dangling bond. Concerning the dangling bond energy, Schröter and Cerva point out that various theoretical calculations obtained values ranging from $0.5 \mathrm{eV}-2.1 \mathrm{eV}$ [114].

In sum, for the first complex of the nucleation path without pre-existing substitutional nickel, the formation enthalpy can be estimated to be between $\Delta H=1.2 \mathrm{eV}$ and $\Delta H=2.8 \mathrm{eV}$. For the first complex of the nucleation path with pre-existing substitutional nickel, including one additional Ni-Si-bond, the total formation enthalpy can be estimated to be between $\Delta H=-0.9$ and $\Delta H=0.9 \mathrm{eV}$.

The considered values cover the experimentally determined difference between the nucleation barrier with and without substitutional nickel of $1.7 \mathrm{eV}$. Low barriers would even imply that the initial complexes could form in equilibrium in certain concentration at the high in-diffusion temperatures. This would potentially promote the formation of clusters as sugested in sec. 3.2.2.

In addition, considering further energy corrections from configurational entropy, hybridization, possible Jahn-Teller relaxations or entropy contributions 
due to changes in the phonon spectrum implies further energy lowering. The numbers of complexions for the complexes formed without and with preexisting $\mathrm{Ni}_{\mathrm{s}}$ is $\Omega_{\text {without }}=4$ and $\Omega_{\text {with }}=12$, resulting in an energy lowering of

$$
k T \ln \left(\Omega_{\text {without }}\right)=0.14 \mathrm{eV} \text { and } k T \ln \left(\Omega_{\text {with }}\right)=0.25 \mathrm{eV}
$$

at $T=900{ }^{\circ} \mathrm{C}$. Energy lowering from hybridization, e.g. studied at the reactivity of metallic surfaces ([115]), can generally be on the order of some tenth eV. Jahn Teller relaxations (as described by Watkins [116]) potentially result in similar corrections. 


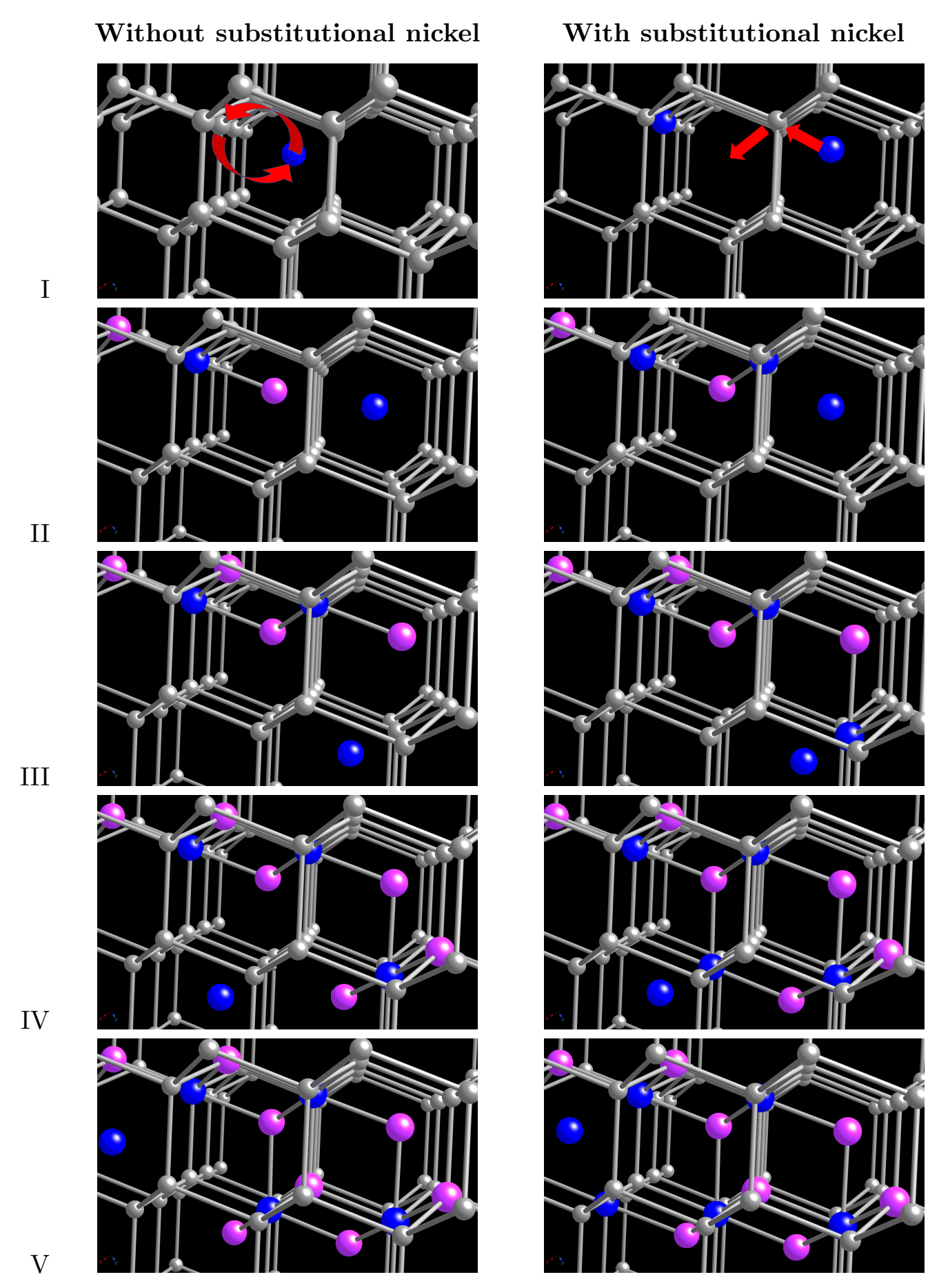

Figure 3.28: Early stages of nickel disilicide nucleation in a perfect crystal (left) and at a substitutional nickel atom (right). The atoms of the silicon host lattice are displayed in grey, involved silicon atoms are highlighted in pink and nickel atoms are blue. 


\section{Growth path}

Further conceivable growth stages of a consecutive growth path are shown in fig. 3.28. They are formed by adding $\mathrm{NiSi}_{2}$ units under completing as many Ni-Si-bonds as possible. The number of Ni-Si-interbonds, added within the corresponding growth step, are given in table 3.3 for nucleation without substitutional nickel and in table 3.4 for nucleation with substitutional nickel. One can imagine that with increasing number of nickel atoms, the number of complexions increases.

The question, if the early stages have indeed planar shape, parallel to $\{111\}$ planes, can not definitely be answered. It is even the case that for precipitates growing out of $\{111\}$-planes the numbers of complexions are by trend higher. However, it seems plausible that the planar structures in $\{111\}$-planes have the lowest energy and are favored for the subsequent transition to a stacking fault to enable the fastest relaxation rate during further growth [9].

The planar growth of early stages has also been observed in other systems: Silide platelets of cobalt have been observed by Utzig [117]. The platelet growth of precipitates has been observed earlier for oxygen [118]. The oxide platelets described there subsequently undergo a morphological transformation from a strain-free to a strained state. A similar effect of planar growth has been observed for copper precipitates at grain boundaries. They have been found to initially form coherent layers in the $\mathrm{Cu}_{3}$ Si-equilibrium phase [119].

\begin{tabular}{|c|c|}
\hline \# Stage & \# Ni-Si-interbonds \\
\hline $\mathrm{I} \rightarrow \mathrm{II}$ & 0 \\
$\mathrm{II} \rightarrow \mathrm{III}$ & 1 \\
$\mathrm{III} \rightarrow \mathrm{IV}$ & 1 \\
$\mathrm{IV}-\rightarrow \mathrm{V}$ & 2 \\
\hline
\end{tabular}

Table 3.3: Number of interbonds formed during the initial reactions of the proposed nucleation path without pre-existing substitutional nickel.

\begin{tabular}{|c|c|}
\hline \# stage & \# Ni-Si-interbonds \\
\hline $\mathrm{I} \rightarrow \mathrm{II}$ & 1 \\
$\mathrm{II} \rightarrow \mathrm{III}$ & 1 \\
$\mathrm{III} \rightarrow \mathrm{IV}$ & 2 \\
$\mathrm{IV} \rightarrow \mathrm{V}$ & 1 \\
\hline
\end{tabular}

Table 3.4: Number of interbonds formed during the initial reactions of the proposed nucleation path with pre-existing substitutional nickel. 


\section{Summary}

The here proposed model qualitatively describes an increased nucleation in the presence of substitutional nickel, suggesting that the critical nucleus may be constituted by substitutional nickel or at least a very small number of $\mathrm{NiSi}_{2}$ units. Against this background, the model can be considered as a beneficial approach to $\mathrm{NiSi}_{2}$-nucleation, providing an idea of the initial stages in $\mathrm{NiSi}_{2}$ precipitation kinetics. A sketch of the precipitation process is depicted in fig. 3.29 .

\section{$\mathrm{NiSi}_{2}$ nucleation scheme}

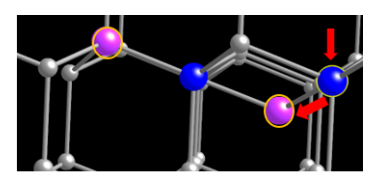

Preferred nucleation at substitutional nickel ( $\left.\Delta \mathrm{H}^{\sim 2 \mathrm{eV}}\right)$.

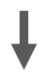

$\mathrm{Ni}_{\mathrm{s}} \mathrm{Ni}_{\mathrm{i}}$ (Lemke, 1996), probably invisible.

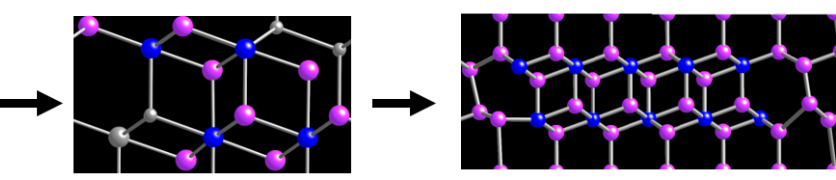

Path of planar growth.

Transition to stacking fault, further growth under fastest relaxation rate (Seibt, 1989).

3-dimensional agglomerates, probably dissolve in subsequent growth.

Figure 3.29: $\mathrm{NiSi}_{2}$-nucleation model 


\subsection{Distribution and electronic properties of individual precipitates}

$\mathrm{NiSi}_{2}$ precipitates formed by immediate undercooling of a solid solution of interstitial nickel in silicon, grown in a self-aligned process with a thickness of only two monolayers [9], represent the thinnest nickel silicide structures that have been observed yet with enormous potential for nanoelectronic applications. Whereas previous electrical analyses mainly probed integral properties from averaging over ensembles of small platelets [69, 10, 71, 73], the current investigation aims to approach the properties of individual structures. Platelets with large diameters, representing buried ultrathin $\mathrm{NiSi}_{2}$ platelets with extraordinary aspect ratio, enabled to access distinctive interface properties.

The growth of these large structures, as sketched in the block diagram in fig. 3.30, was realized by precipitation from high interstitial nickel concentrations under in-diffusion temperatures around $T_{\text {indiff }}=1100^{\circ} \mathrm{C}$. It is assumed that under these conditions, the grown-in vacancies have annealed and equilibrium concentrations for substitutional and interstitial nickel are established. In a two step quenching procedure - first with a moderate temperature gradient in contact with an aluminum heat sink and then by a fast gradient in ethylene glycol - we established two distinct scales for precipitation within one process, resulting in a coarse pattern of large precipitates overlaid by a fine pattern of small precipitates as from the earlier studies $[9,10]$. The current studies are distinguished from the retrograde melting and liquid precipitation studies [120] at much slower cooling rates.

An overview of the spatial distribution of the precipitates with small and large diameters is given in section 3.3.1. In section 3.3.2 recombination and charge collection properties of individual platelets have been probed with energy-dependent EBIC investigations in planar emitter geometry. Finally, in section 3.3.3, charge transport properties along the structures are inspected with high magnification EBIC profiles in cross section geometry.

\subsubsection{Spatial distribution of precipitates}

During the quenching process, interstitial nickel equilibrates at the nickel disilicide boundary layer, by which its concentration and supersaturation level near the boundary layer decrease and the formation of precipitates is hampered in that region [121]. In a simulated concentration profile, fig. 3.32, after 1 second of moderate cooling, the decrease in the concentration of interstitial nickel is roughly extended to a distance of about $d=100 \mu \mathrm{m}-150 \mu \mathrm{m}$ from the boundary layer.

In good correspondence, a Light-Beam-Induced Current (LBIC) overview of a 


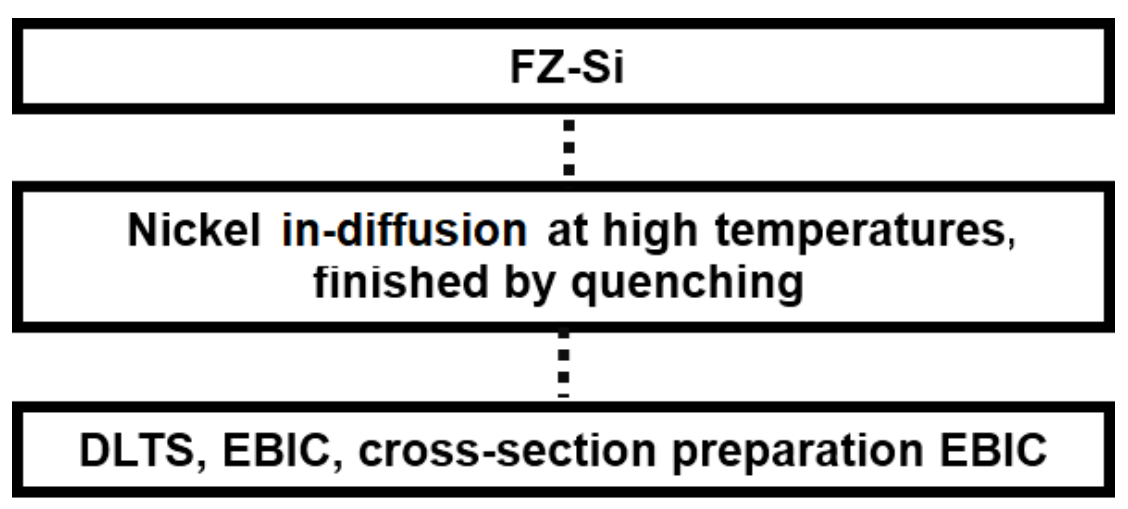

Figure 3.30: Block diagram, showing the preparation for the analysis of large precipitates. The in-diffusion temperature was $T_{\text {indiff }}=1100^{\circ} \mathrm{C}$. The largest precipitate diameter was achieved in our experiments by a subsequent two step quenching for $5 \mathrm{~s}$ in contact with an aluminum heat sink and then in ethylene glycol.

bevel cut, fig. 3.33, shows that the large precipitates have grown in distances $d \geq 130 \mu \mathrm{m}$. The large precipitates appear here as local bright contrasts, as will be discussed in detail in section 3.3.2 and section 3.3.3. Closer to the boundary layer from former nickel in-diffusion, in distances $20 \mu \mathrm{m} \leq d \leq 130 \mu \mathrm{m}$, a homogenously reduced LBIC signal from a minority carrier diffusion length of $L=15 \mu \mathrm{m}$ indicates small precipitates (compare section 1.3.2) from relaxation of interstitial nickel during the second quenching period, roughly down to a distance $d \geq 20 \mu \mathrm{m}$ from the former boundary layer. Their characteristic DLTS-signature is shown in a depth-dependent representation in fig. 3.34. In parallel samples, it was shown that the kinetics of the formation of small precipitates - under sufficient excess concentrations of interstitial nickel - work even below $650{ }^{\circ} \mathrm{C}$. The bright region in distances $d \geq 20 \mu \mathrm{m}$ indicates a region virtually free of precipitates.

An EBIC micrograph, fig. 3.31, of the remote bulk regions in a sample prepared under comparable quenching conditions shows large precipitates, visualized here as line type structures, and small precipitates, visualized as small dark spots, in the bulk regions in-between. According to the situation at the boundary layer, the individual vicinities of the large precipitates, marked by re circles here are by trend free of small precipitates, which can be understood when assuming a lowering of the nickel concentration in their vicinities by internal gettering, as e.g. similarly observed for liquid precipitates by the Buonassisi group [120].

Preferential etching of the surfaces visualizes haze formation apart from the nickel disilicide boundary layer, as illustrated in fig. 3.35, which is in accordance with findings by Seibt and Graff [110] of haze formation with cobalt, nickel, copper and palladium. 


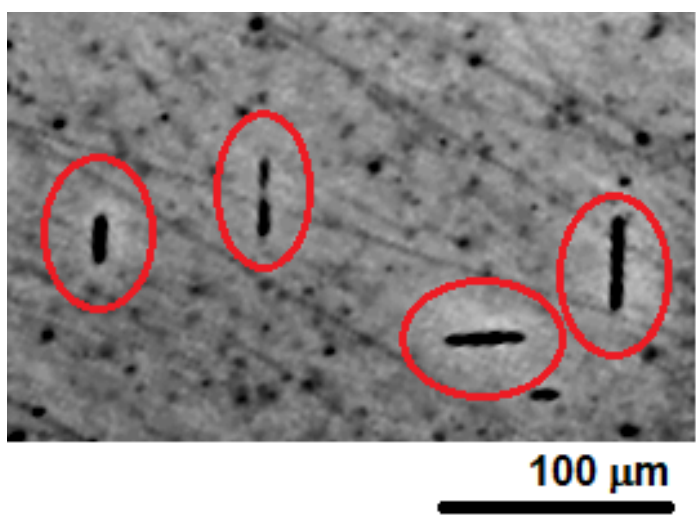

Figure 3.31: EBIC micrograph, showing precipitates with large diameters, identified by dark lines, and small precipitates, identified by dark spots. The vicinities of the large precipitates, marked with red ellipses, are by trend free of small precipitates.

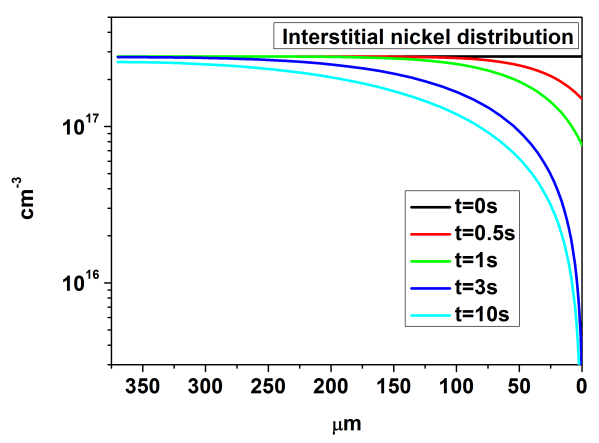

Figure 3.32: Simulated profile of interstitial nickel during the applied quenching procedure.

\section{Summary}

Precipitates have been established in a certain two step quenching procedure. In the bulk regions - apart from the influence of the wafer surfaces - a small density of large precipitates has probably been formed during early cooling stages, and a large density of smaller precipitates in-between from later cooling stages. The direct vicinity of the large precipitates is denuded of small precipitates. 

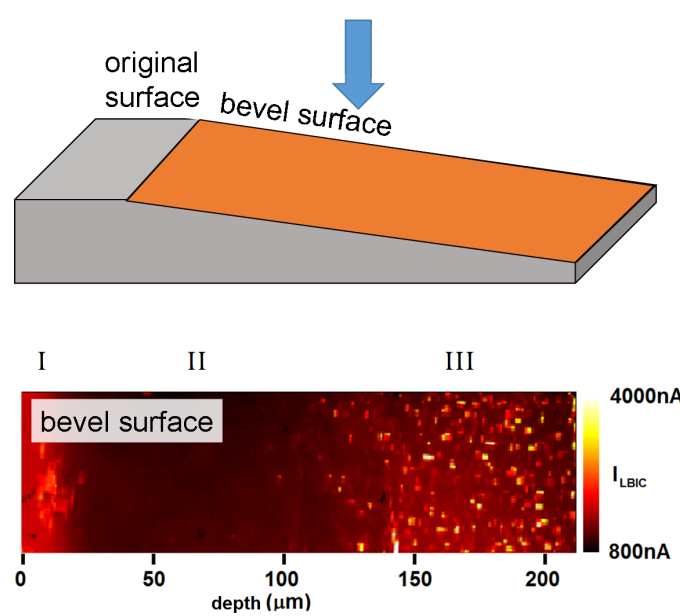

Figure 3.33: Top: Sketch of the sample with bevel surface. Bottom: LBIC overview, revealing different depths below the former nickel in-diffusion layer. Three regions can be distinguished: I) Zone close to the former in-diffusion layer, denuded from recombination active defects, reaching to a distance of about $20 \mu \mathrm{m} \quad$ II) Zone with homogeneously distributed recombination active centers in accordance with small $\mathrm{NiSi}_{2}$ - precipitates III) In distances $\geq 120 \mu \mathrm{m}$ : Additional large structures associated with large diameter $\mathrm{NiSi}_{2}$ precipitates.

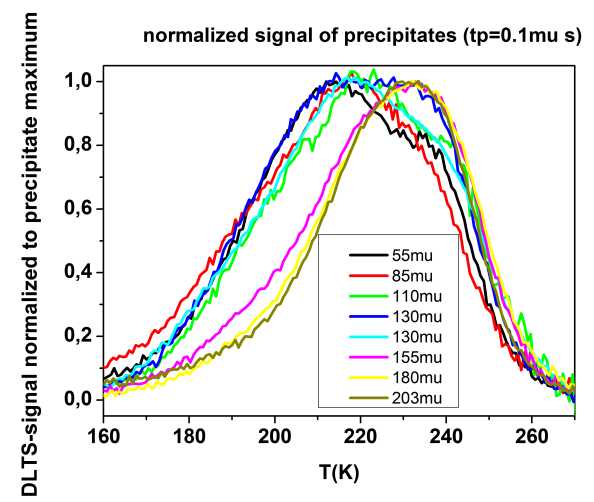

Figure 3.34: Normalized DLTS signature of the precipitates in different distances to the nickel disilicide in-diffusion layer. The width of the spectra indicates by trend increasing diameter of the precipitates with increasing distance. 

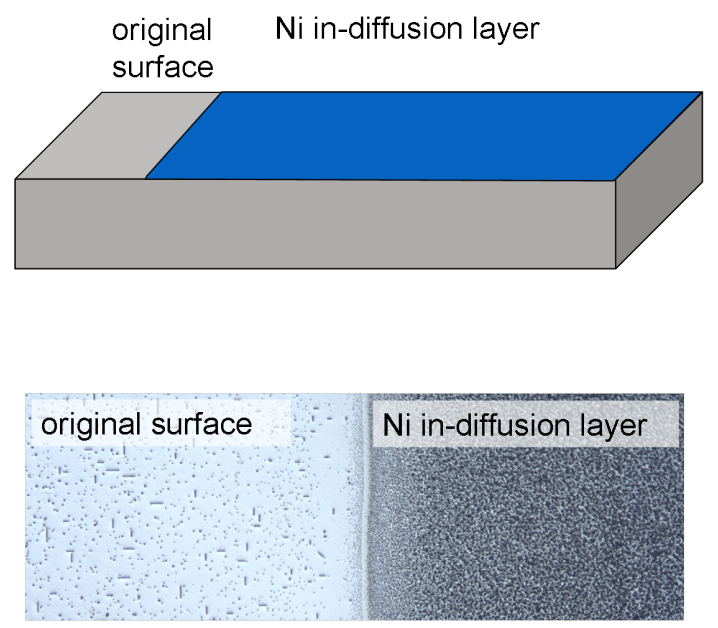

$\overline{100 \mu \mathrm{m}}$

Figure 3.35: Top: Sketch of the sample design after nickel in-diffusion. Bottom: Wafer surface with original surface and nickel in-diffusion layer after Secco selective defect etching. At the original surface, line structures indicate the intersection of platelets with the etched plane, revealing large precipitates at the original surface, in analogy to the earlier observed haze formation [110]. 


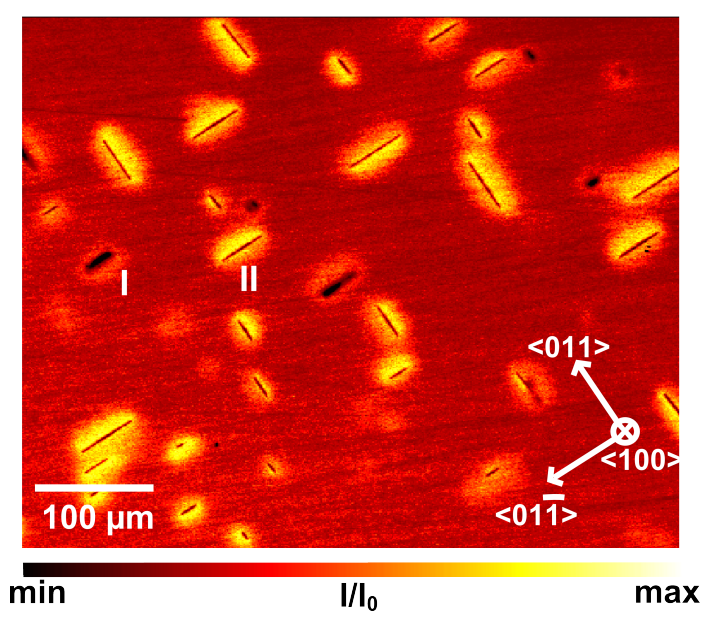

Figure 3.36: Low magnification EBIC-viewgraph of large $\mathrm{NiSi}_{2}$-platelets in edge view, taken with $15 \mathrm{kV}$ beam energy. Two types of profile can be distinguished: one (I) with a pronounced dark core (revealing decreased signal with respect to the bulk regions) and with a slightly bright surrounding (revealing increased signal) and the other (II) with a dominating bright signal, streaked by a narrow dark line.

\subsubsection{Electron beam induced current profiles of individual large precipitates}

A typical set of large $\mathrm{NiSi}_{2}$ platelets in edge-on view is shown in an EBICmicrograph in fig. 3.36. The platelets are silhouetted against the homogeneous background from finely dispersed recombination centers. Obviously, the same type of platelet shows two distinct types of EBIC-profiles, marked as I and II. Profiles of type I are characterized by a center with decreased signal and a surrounding with slightly increased signal. Profiles of type II preponderantly have an increased signal. In the following, decreased signal will be used equivalently with dark contrast; increased signal will be used equivalently with bright contrast. 


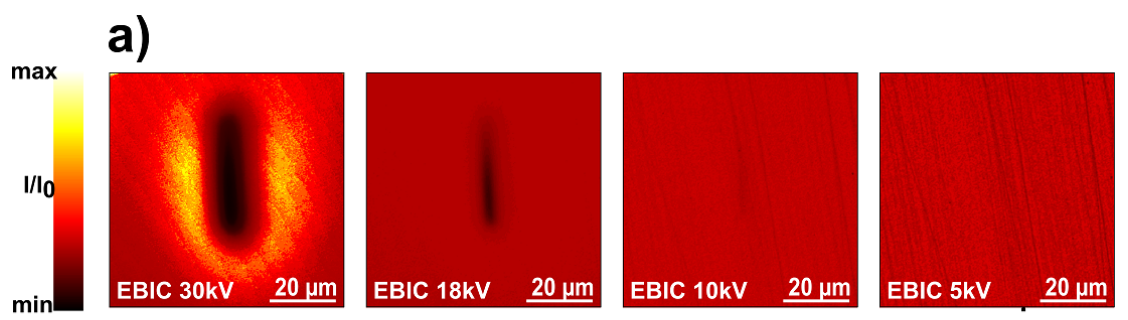

b)

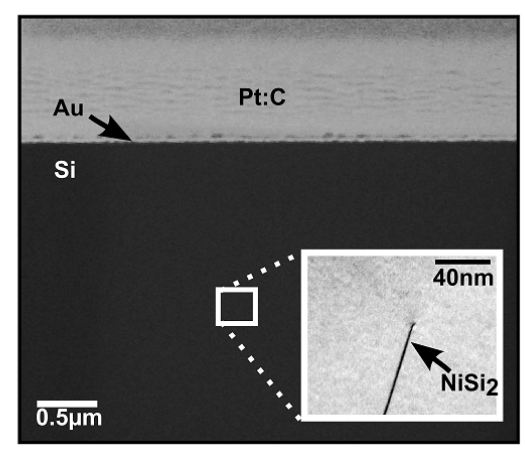

c)

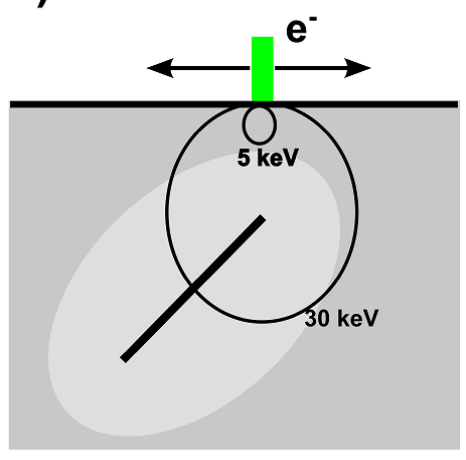

Figure 3.37: a) EBIC image series of $\mathrm{NiSi}_{2}$ platelet that is governed by a dark recombination contrast in its center region. (Type I in fig. 3.36). Additionally, large generation depths reveal a bright contrast in the surrounding of the platelets indicating a denuded zone. Towards lower probing depths, the profile gradually vanishes. The color range for the different beam energies is aligned at the respective background signal. Subsequent cross-section-views (b) by SEM and conventional TEM (inlet) (schematically illustrated on the right) show that the precipitate ( $\left.\mathrm{NiSi}_{2}\right)$ is completely situated inside the silicon bulk $(\mathrm{Si})$. The determined distance to the front contact $(\mathrm{Au})$ is $1.7 \mu \mathrm{m}$. For FIB-preparation a platinum and carbon containing composite (Pt:C) had been standardly deposited on top, to protect nearby regions against the gallium ion beam. The geometry is sketched in (c).

\section{Non-contact geometry}

Structures of profile I are completely situated inside the sample. A typical precipitate with such a profile is depicted in fig. 3.37a) in an EBIC image series, taken at beam energies from $30 \mathrm{kV}$ down to $5 \mathrm{kV}$ with probing depths between $\mathrm{d}_{30}=6,54 \mu \mathrm{m}$ and $\mathrm{d}_{5}=0.28 \mu \mathrm{m}$. Exemplary line profiles are shown in fig. 3.38.

The dark contrast for large probing depths in the core of the precipitate indicates efficient capture of minority carriers in a Coulomb potential and recombination via bandgap states, which is in accordance with the pronounced recombination activities $[75,109,16,76]$ at bulk-shaped (three dimensional) transition metal silicide precipitates. The contrast values of 0.4 are comparable to those by Kittler et al. [75] at small precipitates after ripening. It can be clearly seen that the dark contrast at the current structures is related to the 


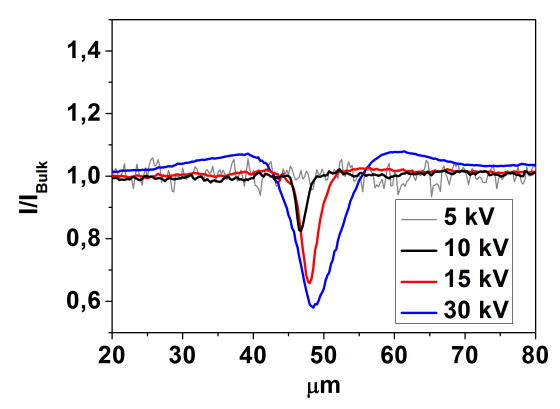

Figure 3.38: Exemplary EBIC line profiles, taken with different probing depths, across a large precipitate in non-contact geometry

interface regions which indicates that the recombination is mainly determined by deep states occurring at the interfaces. This could be explained by the model comprehending interface states, proposed in section 1.3.1. Additionally, recombination may occur at interfacial defects at dislocations or terraces at occasional faceted regions.

In addition, an increased signal is observed in the surrounding of the precipitates. The extension of the corresponding regions exceeds the space charge width discussed in the following sections 3.3.2and 3.3.3. It rather indicates zones around the precipitates, which are denuded of recombination active bulk defects. The zones are in accordance with the internal gettering regions, described in section 3.3.1, formed during the growth of the silicide precipitates as sites for local gettering [122]. From the profiles, the corresponding local gettering range is estimated to be about $20 \mu \mathrm{m}$, which is comparable to the width of a gettering zone at the nickel boundary layer in fig. 3.33. This gives a hint that the large precipitates may form in a very early stage of cooling.

The profile, most pronounced at $30 \mathrm{kV}$, gradually vanishes towards smaller generation depths. At $5 \mathrm{kV}$, the defect contrast is no longer visible, indicating that the complete defect lies deeper than the smallest probed depth. This is confirmed by subsequent cross-section analysis, depicted in the SEM-micrograph in fig. 3.37b) and schematically illustrated in fig. 3.37c). An interspace of $1.7 \mu \mathrm{m}$ as the smallest distance, found between the rim of the platelet and the front surface furthermore clarifies that the structure is separated from the gold contact. 


\section{Contact geometry}

The precipitates of the profile type II are displayed in fig. 3.39, exemplary line profiles are shown in fig. 3.40. Large generation depths reveal a slightly dark line which is far too weak to be explained by recombination losses at the whole platelet and is rather attributed to the intersection with the Au contact. Instead, the entire structure is governed by substantially increased signal, which is far too pronounced to be explained by a denuded zone (compare the line profiles for $30 \mathrm{kV}$ in fig. 3.38 and fig. 3.38). With a gradually diminishing bright signal towards smaller generation depths, the dark line remains visible even in the smallest probing depths. Subsequent SEM- and TEM- cross section views demonstrate that the dark contrast coincides with the intersection of the platelet with the front surface. Under the provision that the platelet electronically couples to the Au emitter, the increased signal implies charge collection at the structure itself and indicates an efficient channel for minority carriers, captured in the space charge region of the structure, to add to the induced current. The bright signal furthermore indicates that the charge collection at the structure is far more efficient than charge collection solely at the gold contact. This can be understood by the highly regular $\mathrm{Si} / \mathrm{NiSi}_{2}$ interfaces of the structure. In comparison, recombination centers seem to be present at the gold contact. This seems plausible when considering a native oxide layer and small scratches that remained from the preparation process without chemical etching.

\section{Summary}

From depth-dependent EBIC profiles can be distinguished between precipitates in non-contact geometry and in contact geometry. Precipitates in noncontact geometry reveal bright contrasts, indicating a denuded zone in their close vicinity, and strong dark contrasts over the whole interface, indicating a high recombination activity at an internal Coulomb barrier. Precipitates in contact geometry only reveal a slight dark contrast at their intersection line, whereas pronounced bright contrasts indicate efficient charge collection at the interfaces and charge transport to the external contact. 
a)
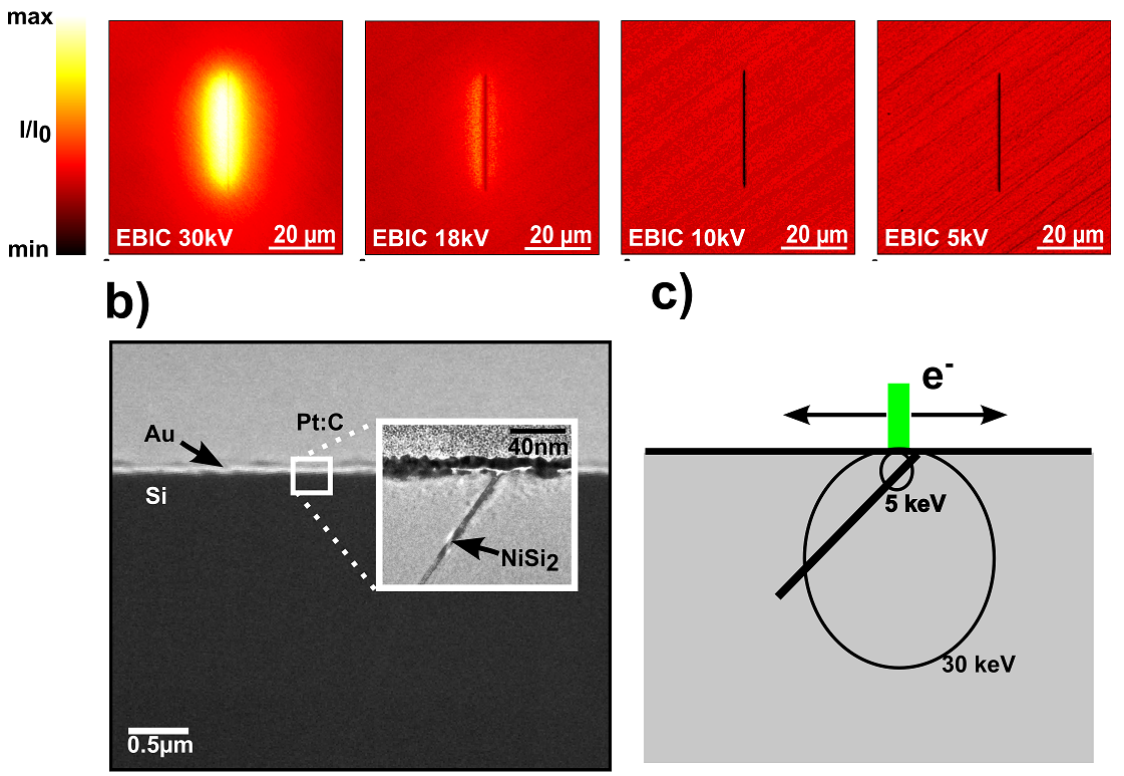

c)

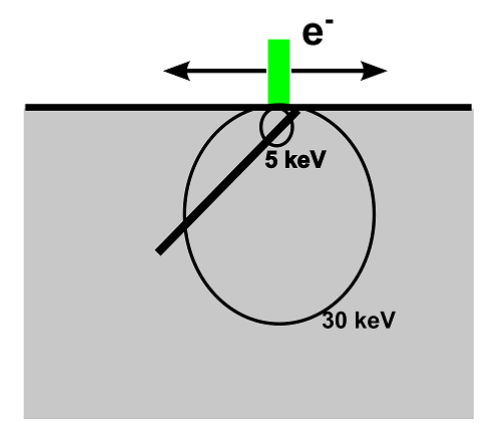

Figure 3.39: a) EBIC image series of $\mathrm{NiSi}_{2}$-platelet intersecting the front surface, taken with different beam energies. At large probing depths, the increased signal at the defect site indicates charge transport along the precipitate. b) Side view by subsequent analysis by SEM and TEM analysis, illustrated in the schematic representation c), indicating that the precipitate is in contact with the emitter at the front surface which offers a channel for minority carriers, generated in deeper sample regions $(30 \mathrm{kV})$ to add to the external current.

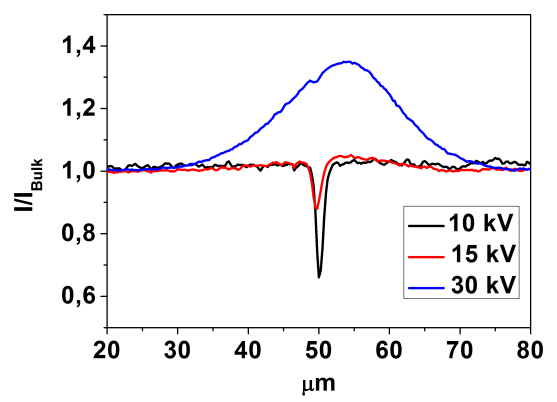

Figure 3.40: Exemplary EBIC line profiles, taken with different probing depths, across a large precipitate in contact geometry. 


\subsubsection{Charge collection profiles of individual large precipitates at FIB-prepared cross-sections}

In the following, specific aspects of the charge transport at large $\mathrm{NiSi}_{2}$ precipitates in contact geometry are accessed in cross-sectional studies. For this purpose, structures are cut, as illustrated in fig. 3.41, by Focused Ion Beam preparation with high energetic gallium ions, starting with $30 \mathrm{kV}$ and $7 \mathrm{nA}$ for a rough removal of the material and finishing with $5 \mathrm{kV}$ and $29 \mathrm{pA}$. For the subsequent SEM and EBIC analysis, the incident beam is tilted to the precipitate plane by an angle of $35^{\circ}$ which results in a broadening of the EBIC signal and benefits the resolution of the precipitate line.
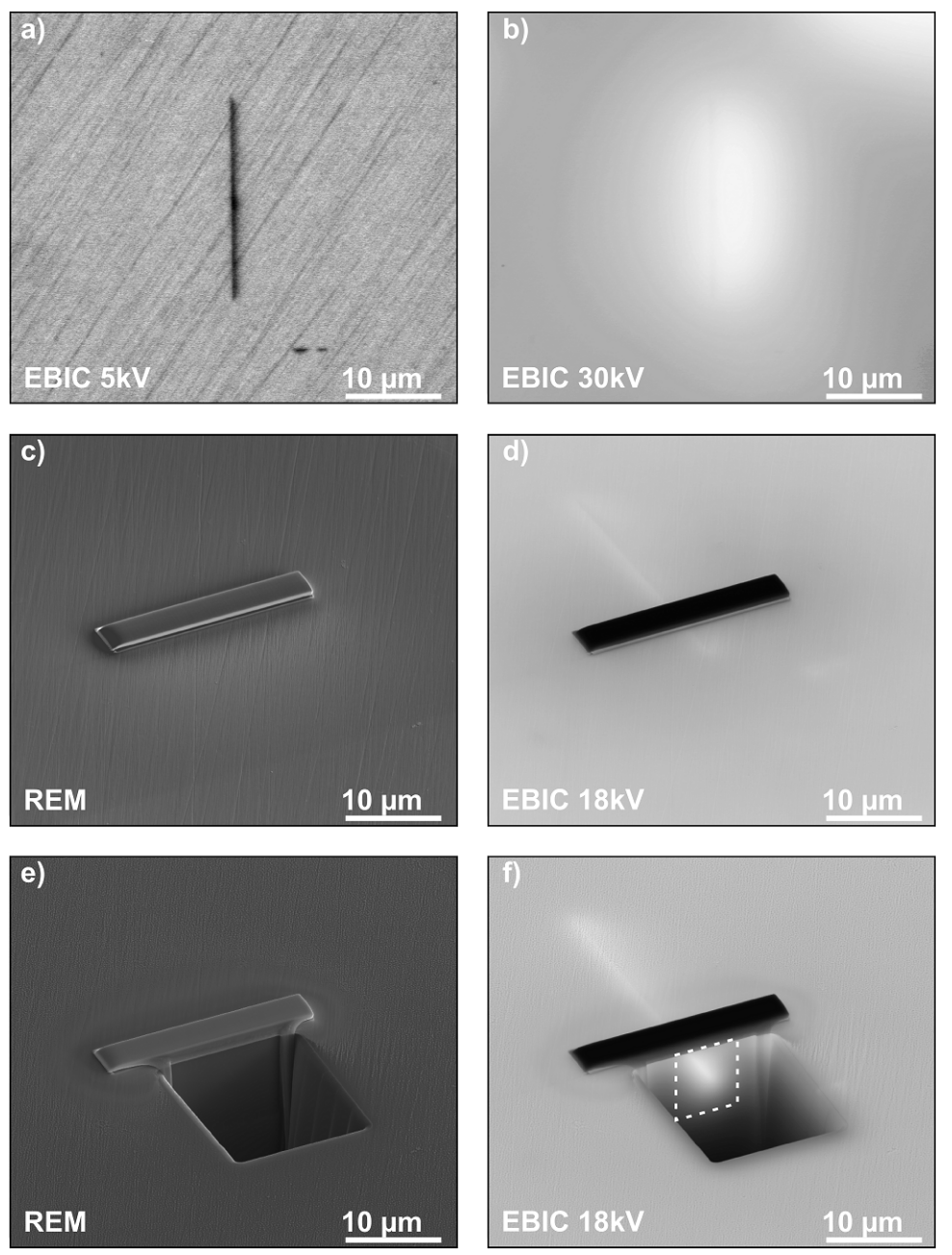

Figure 3.41: SEM and EBIC images during the preparation of cross sections. (a),(b): top view of a large precipitate in contact geometry. (c),(d) inclined and rotated view of the same feature, after deposition of a platinum and carbon containing composite layer to protect the regions of interest against the gallium beam during subsequent milling of the cross section plane, shown in (e) and (f). 


\section{Overview}

A cross section of a typical $\mathrm{NiSi}_{2}$ platelet coupling to the gold contact is presented in fig. 3.42. The structure, displayed in SEM (a) by a slight bright contrast, is recognized in EBIC (b) by a dark line that is framed by a bright signal over its entire length of $3.3 \mu \mathrm{m}$ into the sample. The subsequent TEM images (c) and (d) show a thickness of only two $\mathrm{NiSi}_{2}$ layers in the typical configuration with coherent interfaces over large parts. Occasional strain contrasts (marked with black circles) at the interface indicate in-plane dislocations. 


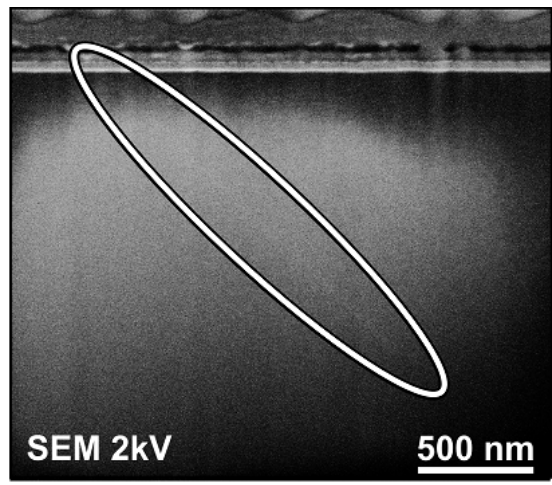

(a)

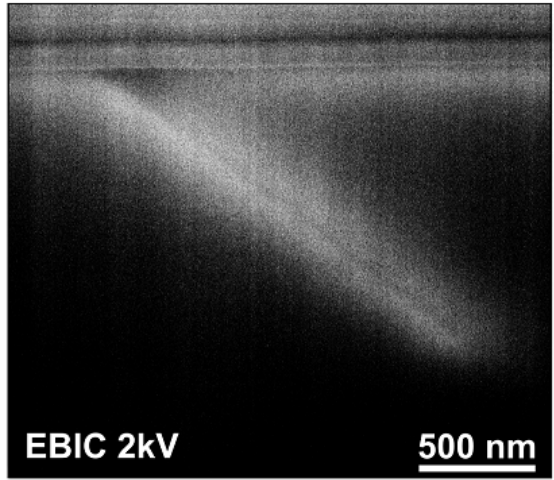

(b)

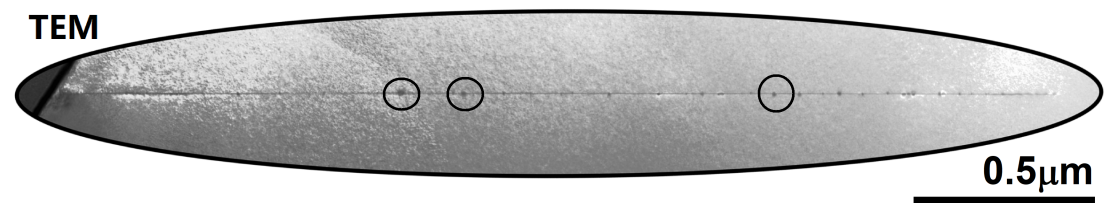

(c)

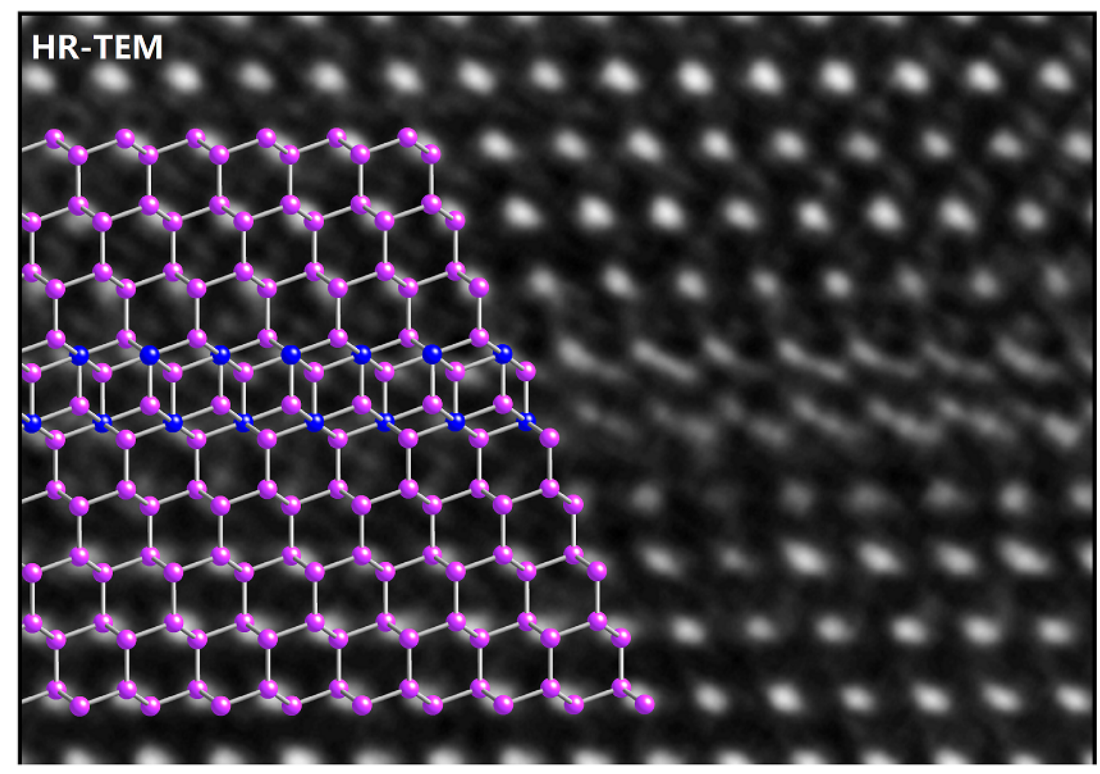

(d)

Figure 3.42: Analysis of a $\mathrm{NiSi}_{2}$-structure in cross section view. In a high magnification SEM image (a) the structure can be seen as a slightly bright line, marked here by an ellipse. In the corresponding EBIC micrograph (b) the line is surrounded by a bright signal contrast, that demarcates the charge collection region. The dimensions of the dark line in the EBIC micrograph exactly matches to its dimensions in the TEM micrograph (c). The structure reveals irregular strain contrasts which are possibly related to in-plane dislocations, some of which are marked by (circles) here. A lattice image in (110)-projection (d) of a typical region reveals the structure of the thin $\mathrm{NiSi}_{2}$ platelets, as illustrated with the superimposed lattice model. 


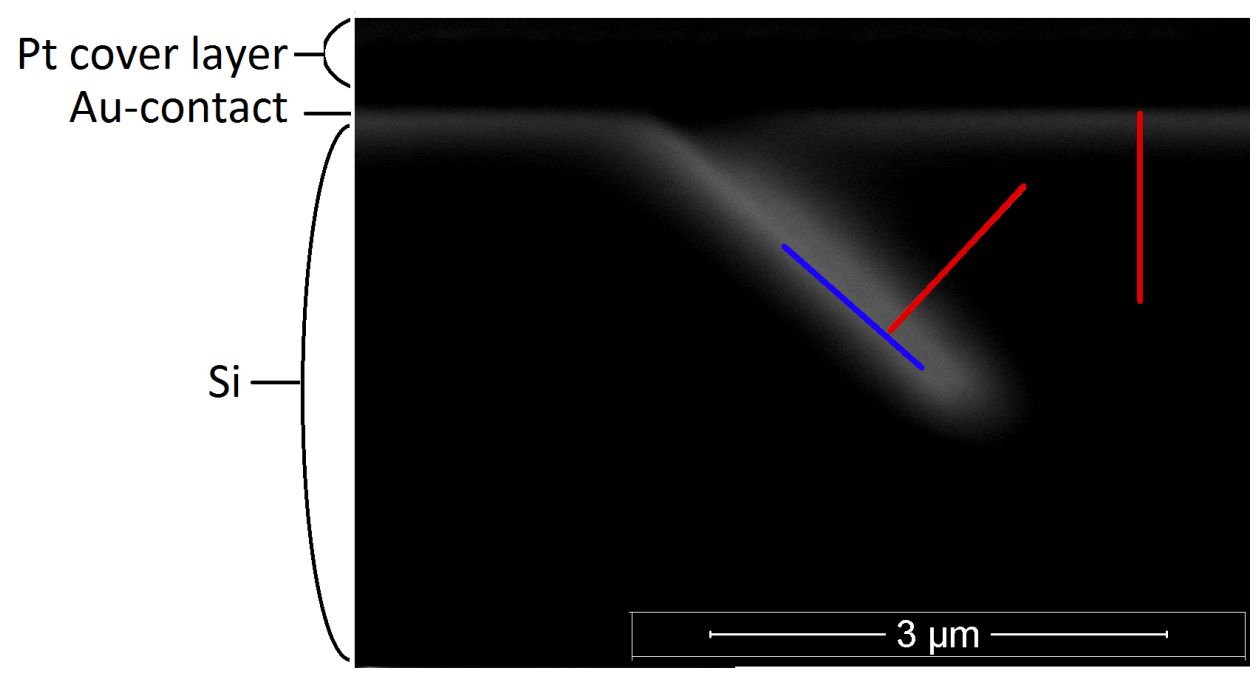

Figure 3.43: Magnified EBIC view of cross section, illustrating the positions of the linescans in the following figs. 3.44 (red) and 3.45 (blue).

\section{Dead layer}

A peculiarity of EBIC measurements at FIB-prepared structures are recombination processes near the Ion-Beam-exposed surfaces due to ion beam damage effects [123]. In Peretzki et al. [124] these are described by a dead layer. In the magnified EBIC image in fig. 3.43, the dead layer leads to zero current at the cross section surface away from charge collecting structures. In the current case of roughly 15 micrometer bulk diffusion lengths (section 3.3.1), the corresponding loss of minority carriers to the FIB-prepared surface is the dominant recombination process. Narrowing the charge collection range around the examined structure, it is thus a prerequisite to resolve the charge collecting structures in the sub-micrometer range.

\section{Space charge region imaging}

Especially for shallow generation depths, it is reasonable to assume that the width of the charge collection function across the structure is closely linked to the width of the space charge region. For the generation with a beam energy of $2 \mathrm{kV}$, as for the magnified image in fig. 3.43, with a primary electron range of $57 \mathrm{~nm}$ (equation 2.17 in section 2.3), most of the generation volume is in the dead layer [125] and only a small fraction of charge carriers is generated in the bulk regions below. The beam width, determined from the resolution of an edge, is $d_{\text {beam }}=20 \mathrm{~nm}$. Based on this, the generation width is $60 \mathrm{~nm}$. A comparison of line profiles across the gold Schottky contact and across the structure to one side, shown in fig. 3.44, reveals a similar extension. This implies that 


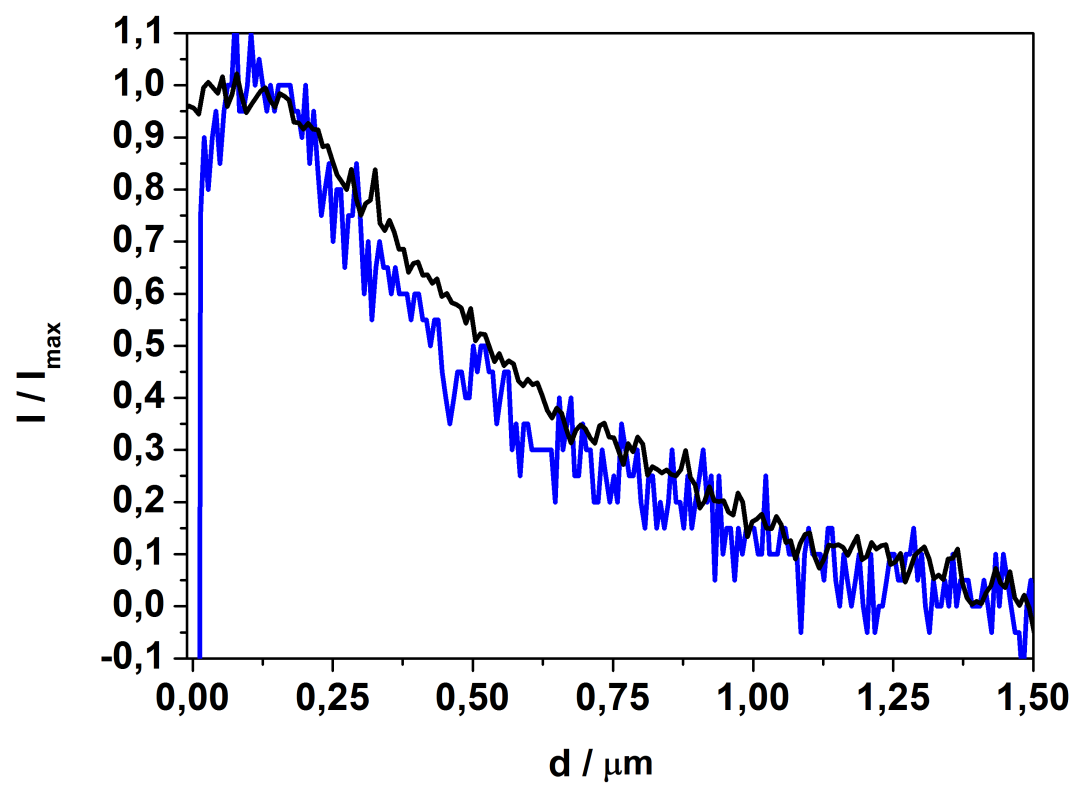

Figure 3.44: Profiles across the space charge regions of the Au-contact (blue) of the $\mathrm{NiSi}_{2}$-structure (black) to one side, according to the positions, marked in fig. 3.43. Both profiles show the same shape.

the potential barriers of the gold Schottky contact and the $\mathrm{NiSi}_{2}$ precipitate are comparable. In accordance with typical Schottky barrier heights at gold contacts (standardly determined at gold contacts for DLTS measurements in this work), one can deduce a Schottky barrier height of about $0.7 \mathrm{eV}$. This is in accordance with Schottky barrier heights determined at $\mathrm{NiSi}_{2}$ layers by Tung et al. [12] of $0.65 \mathrm{eV}$ or $0.79 \mathrm{eV}$. The corresponding charge density can be determined according to the electrostatic relations of a Schottky barrier [79], which reveals $\rho_{\text {area }}=2 \cdot 10^{11} \mathrm{~cm}^{-2}$. The net charge density in relation with charge transport along the structure (compare sec. 3.3.2) imply the presence of charges at the interface in connection with partially filled lower dimensional bands. These can be related to the interfacial defects (section 3.3.3) or to [9] sevenfold coordination of nickel atoms at the interface. The charge density derived above is about four orders of magnitude smaller than the concentration of interface atoms [10]. In a recent review by Tung [126], the contribution of the interface states and of the work function to the Schottky barrier is described.

The barrier is higher than the barriers of around $0.4 \mathrm{eV}$ which were found for the precipitates with smaller diameters [71] or [76]. In a qualitative treatment, this becomes plausible from considering the capacitance of a thin oblate ellipsoid in vacuum, based on calculations given in $[127,128]$. For a given charge 
density $\rho=Q /\left(\pi a^{2}\right)$, the potential depends on the diameter in the form

$$
V=\frac{\pi \rho a}{2}
$$

Even taking the screening of the space charge regions into account, one can deduce that the barrier height qualitatively decreases with decreasing diameters, which explains a smaller barrier at smaller precipitates.

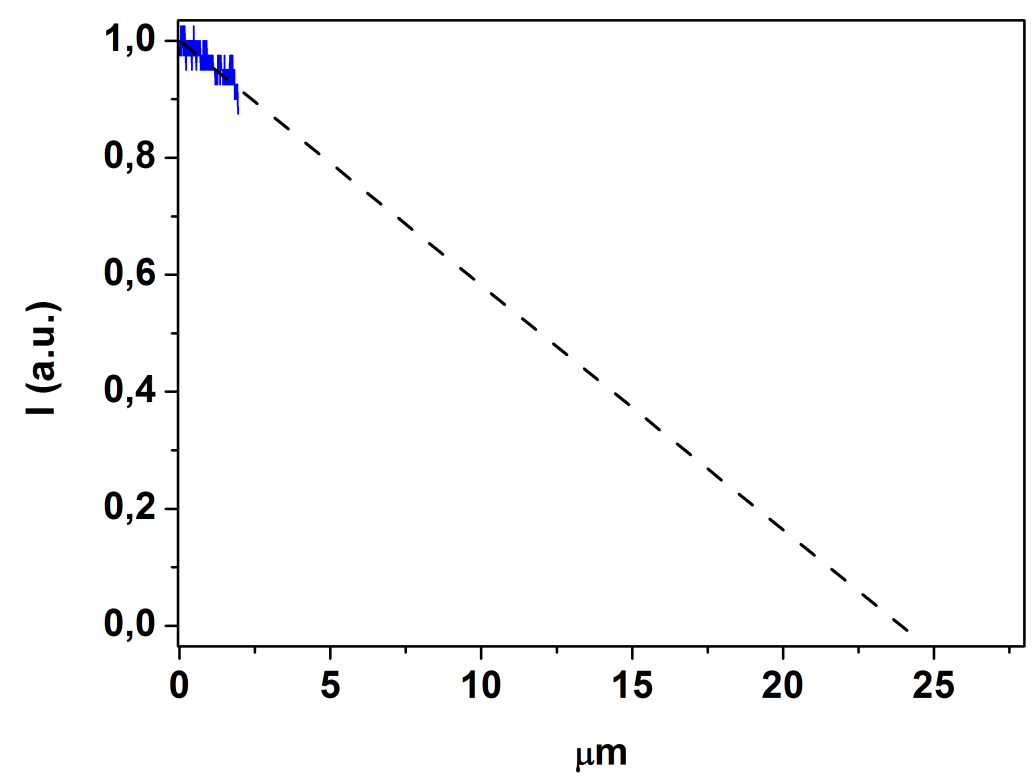

Figure 3.45: Current profile along precipitate (blue). Extrapolation to zero current (black dashed line) reveals a decay length of $24 \mu \mathrm{m}$.

\section{Charge transport along the structure}

The signal along the structure, depicted in fig. 3.45 decreases to zero within a decay length of $24 \mu \mathrm{m}$. The rather small decrease of the current along the structure implies good transport properties. A similar effect has been found earlier [129] at dislocation networks in bonded silicon wafers and at grain boundaries in special multicrystalline silicon [130]. Possible loss mechanisms are due to charge exchange with the adjacent bulk regions.

\section{Maximum current}

At higher injection levels, the charge collection at the $\mathrm{NiSi}_{2}$ structure was more efficient than at the Au-contact. In separate EBIC analyses at a precipitate in 
a ridge (described in more detail in the Appendix A.7), maximum currents of $I_{E B I C}=2.7 \mu \mathrm{A}$ have been detected without damage of the structure. Assuming a thickness of the precipitate plane of $3 \mathrm{~nm}$ and a thickness of the ridge of $10 \mu \mathrm{m}$ this gives a current density of $9 \cdot 10^{7} \mathrm{~A} \cdot \mathrm{m}^{-2}$. We may assume that the EBIC-signal here is limited by the current of the incident beam that is limited here by the electron beam apertures.

A rather small resistivity has been found by Hsin and Deng [131] for ultra-thin epitaxial $\mathrm{NiSi}_{2}$ layers with a value of $\rho_{\mathrm{NiSi}_{2}}=14.93 \mu \Omega \mathrm{cm}$. Assuming this value for the $\mathrm{NiSi}_{2}$ precipitates, one obtains a rather small potential decrease of $12 \mu \mathrm{V} / \mu \mathrm{m}$.

\section{Summary}

The EBIC analysis in cross section geometry substantiates that $\mathrm{NiSi}_{2}$ structures in the contact geometry are an integrated part of the measurement circuit for the induced current, as sketched in fig. 3.46. Charge carriers are generated in the bulk, separated in the space charge region and conducted via the structure contributing to the EBIC signal. A high recombination activity at the FIB-prepared surface enables high magnification EBIC analyses of the precipitate structure. A bright contrast indicates charge collection in the space charge region, which is comparable to that of the gold contact, indicating a Coulomb barrier of roughly $0.7 \mathrm{eV}$. A maximum current density of $9 \cdot 10^{7} \mathrm{Am}^{-2}$ was measured, which was probably limited by the apertures in the applied microscope. Small signal variations along the structure indicate small losses, possibly due to charge exchange with the adjacent bulk regions or leakage currents at the FIB-prepared surfaces.

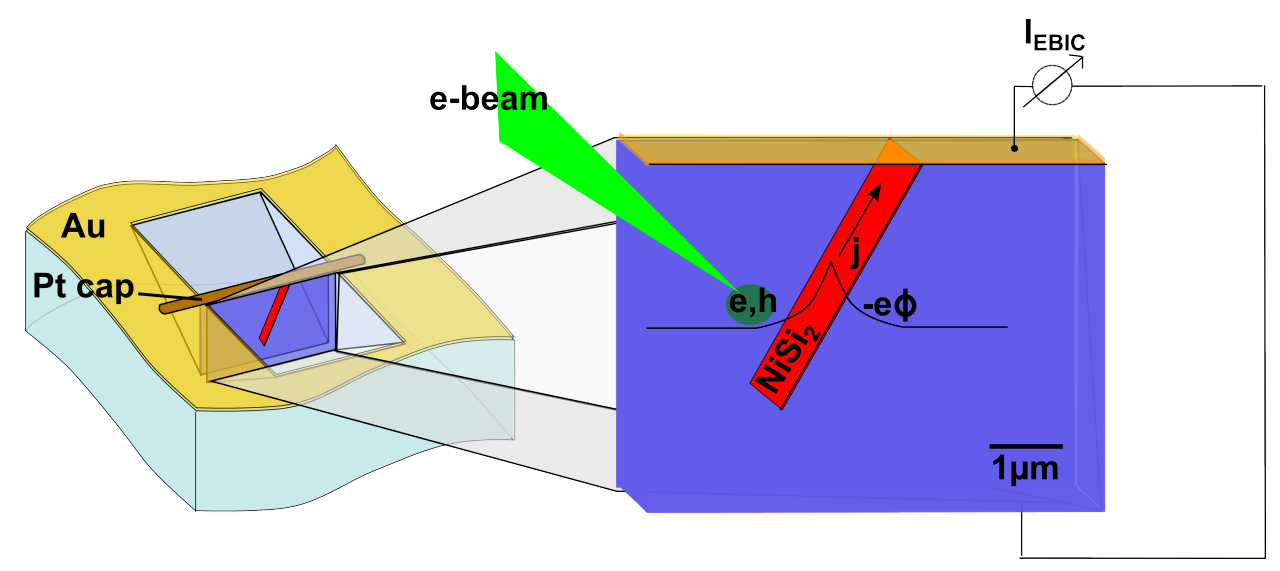

Figure 3.46: Sketch of cross section measurement, shown here for a ridge. 


\section{Chapter 4}

\section{Summary}

Systematic annealing combined with nickel marker in-diffusion provided an access to the annealing kinetics of grown-in vacancy nitrogen related complexes in FZ-silicon. Electrical activation upon annealing was attributed to conversion reactions of the grown-in nitrogen-vacancy related complexes. In addition to an already known deep level (labelled here with X186) of substitutional nitrogen, a second level (X295) of this complex was found near the midgap. Its emission parameters have been derived in an approach, considering balanced majority and minority carrier emission processes.

Nickel in-diffusion detected maximum vacancy concentrations in accordance with the difference in incorporated concentrations of vacancies (V) and interstitials (I) from the crystallization temperature. In the as-grown state, they are supposed to be bound to the nitrogen complex in stochiometries of $\mathrm{NV}_{6}$ or $\mathrm{N}_{2} \mathrm{~V}_{10}$. Upon subsequent wafer-based annealing at temperatures between $T \approx 500{ }^{\circ} \mathrm{C}$ and $T \approx 700{ }^{\circ} \mathrm{C}$ they are found to irreversibly decompose by vacancy release and residuing substitutional nitrogen. With increasing temperatures, all nitrogen-related deep levels and weakly bound vacancy complexes anneal out irreversibly, possibly associated with out-diffusion to the wafer surfaces.

Due to the comparably low binding energies of nickel to vacancies, nickel is a selective marker for weakly bound vacancies. Therefore, the absence of weakly bound vacancies in impure silicon materials indicated the predominance of alternative vacancy reaction paths during crystal growth that result in reactions to stable complexes or interactions with dislocations.

The control of vacancy concentration levels provided an approach to early stages of nickel disilicide nucleation in silicon. The trapping of nickel atoms at weakly bound vacancies has been exploited to explore the effect of substitutional nickel on the nickel disilicide nucleation process. An increased density of precipitates revealed evidence for efficient nucleation at substitutional nickel as nucleation site. The nucleation probability is increased by a factor of about 
$10^{9}$, corresponding to a decrease of the nucleation barrier of $1.7 \mathrm{eV}$.

Upon precipitation, the decrease in substitutional nickel concentration was slightly larger than the resulting precipitate densities. Possibly, some invisible clusters have formed that do not subsequently transform to stacking faults or even completely dissolve in favour of the transformed precipitates.

A bond counting approach qualitatively describes a decreased nucleation barrier in the presence of substitutional nickel, suggesting that the critical nucleus may be constituted by substitutional nickel or at least a very small number of $\mathrm{NiSi}_{2}$-units. Against this background, the model can be considered as a beneficial approach to $\mathrm{NiSi}_{2}$-nucleation, providing an idea of the initial stages in $\mathrm{NiSi}_{2}$ precipitation kinetics.

The analysis of individual $\mathrm{NiSi}_{2}$ precipitates in silicon provides a contribution to probing the downscale limit of $\mathrm{NiSi}_{2}$ structures. Precipitates have been grown in a certain two step quenching procedure. In the bulk regions - apart from the influence of the wafer surfaces - a small density of precipitates with large diameters was probably formed during early cooling stages, and a large density of smaller precipitates in-between from later cooling stages. The direct vicinity of the large precipitates is denuded of small precipitates.

The $\mathrm{NiSi}_{2}$ precipitates with large diameters enabled spatially resolved electrical analyses at individual structures in EBIC studies. Dark contrasts indicated strong recombination activity at the interface. Bright contrasts at the precipitate coupling to an external contact indicated efficient minority charge capture at the $\mathrm{Si}-\mathrm{NiSi}_{2}$ interface and charge transport along the ultrathin structures. Cross section profiles indicated a Coulomb barrier of roughly $0.7 \mathrm{eV}$. A maximum current density of $9 \cdot 10^{7} \mathrm{Am}^{-2}$ was measured. The EBIC signal was probably limited here by the current of the incident beam which was limited by the apertures in the applied microscope. A slight signal decrease along the structure indicates small losses, probably due to charge exchange with the adjacent bulk regions. 


\section{Appendix A}

\section{Appendix}

\section{A.1 The equation system for nickel diffusion in silicon}

\section{A.1.1 Diffusion reaction kinetics}

The exchange of foreign atoms between interstitial and substitutional sites involves the interaction with intrinsic point defects, for which two underlying mechanisms are considered: One describes the formation of a substitutional atom by the reaction of an interstitial atom with a vacancy. The concept was derived by Frank and Turnbull[132] for the analysis of the diffusion of copper in silicon. The reaction equation of the dissociative mechanism or mechanism is

$$
M_{i}+V \rightleftharpoons M_{s}
$$

The second mechanism, proposed by Gösele et al. [133] to explain the diffusion profiles for gold in silicon, assumes that an interstitial atom occupies a substitutional site by generating a silicon self interstitial. The corresponding reaction equation of the kick-out mechanism is given by

$$
M_{i} \rightleftharpoons M_{s}+I,
$$

which is designated as Kick-out mechanism. In the case of nickel diffusion, this mechanism plays only a minor role. Only for the sake of completeness, it is taken into account here. All described reactions are combined in the following diffusion reaction equation system. It represents a set of continuity equations, one for each species. For a one dimensional geometry with the space coordinate $\mathrm{x}$ one obtains 


$$
\begin{aligned}
\frac{\partial C_{N i_{s}}}{\partial t}= & k_{F T \rightarrow} C_{N i_{i}} C_{V}-k_{F T \leftarrow} C_{N i_{s}}+k_{K O \rightarrow} C_{N i_{i}}-k_{K O \leftarrow} C_{I} C_{N i_{s}} \\
\frac{\partial C_{N i_{i}}=}{\partial t}= & D_{N i_{i}} \frac{\partial^{2} C_{N i_{i}}}{\partial x^{2}}-\frac{\partial C_{N i_{s}}}{\partial t} \\
\frac{\partial C_{V}}{\partial t}= & D_{V} \frac{\partial^{2} C_{V}}{\partial x^{2}}+k_{F T \leftarrow} C_{N i_{s}}-k_{F T \rightarrow} C_{N i_{i}} C_{V} \\
& -k_{B}\left(C_{I} C_{V}-C_{I}^{e q} C_{V}^{e q}\right)-k_{B V}\left(C_{V}-C_{V}^{*}\right) \\
\frac{\partial C_{I}}{\partial t}= & D_{I} \frac{\partial^{2} C_{I}}{\partial x^{2}}+k_{K O \rightarrow} C_{N i_{i}}-k_{K O \leftarrow} C_{I} C_{N i_{s}}-k_{B}\left(C_{I} C_{V}-C_{I}^{e q} C_{V}^{e q}\right) \\
& -k_{B I}\left(C_{I}-C_{I}^{*}\right) .
\end{aligned}
$$

The equation system includes the Frenkel-reaction

$$
V+I \rightleftharpoons 0
$$

and the equilibration of vacancies and self interstitials at sinks, homogeneously distributed in the bulk, as have to be taken into account in the case of high dislocation densities.

\section{A.1.2 Rate constants}

Assuming the reactions to be diffusion-limited (as described in Pichler [54], p.55), the rate constants can be given by

$$
\begin{aligned}
k_{F T \rightarrow} & =4 \pi r_{i V}\left(D_{N i_{i}}+D_{V}\right) \\
k_{F T \leftarrow} & =k_{F T \rightarrow} \frac{C_{N i_{i}}^{e q} C_{V}^{e q}}{C_{N i_{s}}} \\
k_{K O \rightarrow} & =4 \pi r_{i I} D_{N i_{i}} \\
k_{K O \leftarrow} & =k_{K O \rightarrow} \frac{C_{N i_{i}}^{e q}}{C_{N i_{s}}^{e q} C_{I}^{e q}} \\
k_{B} & =4 \pi r_{V I}\left(D_{V}+D_{I}\right) \alpha \\
k_{B V} & =\frac{2 \pi N_{d i s l} D_{V}}{\ln \left(r_{d} / r_{0}^{\prime}\right)} \\
k_{B I} & =\frac{2 \pi N_{d i s l} D_{I}}{\ln \left(r_{d} / r_{0}^{\prime}\right)}
\end{aligned}
$$

$k_{B V}$ and $k_{B I}$ result from a mean-field approach for the annihilation at dislocations with $r_{d}$ the mean distance between the dislocations and $r_{0}^{\prime}$ the (effective) 
capture radius. The equation system is in accordance with [134] The expression $\alpha$ stands for an entropy barrier that slows down the relaxation in equilibrium.

$r_{i V}, r_{i I}, r_{V I}$ denote the capture radii. They are estimated by the nearest neighbor distance in the silicon lattice.

\section{A.1.3 Point defect parameters}

The diffusion coefficients and equilibrium concentrations for vacancies and selfinterstitials are taken from Sinno [113, 135], given by

$$
\begin{aligned}
& C_{I}^{e q}(T)=2.97 \cdot 10^{23} \exp \left(1.40+3.85 \cdot 10^{-3} T\right) \exp \left(-\frac{3.46+3.08 \cdot 10^{-4} T}{k T}\right) \\
& C_{V}^{e q}(T)=4.97 \cdot 10^{22} \exp \left(-3.70+3.53 \cdot 10^{-3} T\right) \exp \left(-\frac{2.48+2.33 \cdot 10^{-4} T}{k T}\right) \\
& D_{I}(T)=0.242 \exp \left(-\frac{0.937}{k_{B} T}\right) \mathrm{cm}^{2} / \mathrm{s} \\
& D_{V}(T)=10^{-3} \exp \left(-\frac{0.457}{k_{B} T}\right) \mathrm{cm}^{2} / \mathrm{s} .
\end{aligned}
$$

They had been obtained from a single set of crystal growth experimental data and have been verified for a wide range of growth conditions with a high accuracy. 


\section{A.2 Determination of the free charge carrier capture kinetics by Pons[80]}

\section{A.2.1 Small deep trap concentrations}

According to the work by Pons [80], the DLTS-amplitude is given by

$$
\frac{\Delta C\left(t_{p}\right)}{C_{0}}=\frac{1}{2} \frac{N_{T}}{N_{D}} R\left(t_{p}\right) \times\left[\left(1-\frac{L_{0}}{W_{0}}\right)^{2}-\left(\sqrt{1-\frac{\Delta V}{\left|V_{0}\right|-\frac{k T}{q}}}-\frac{L_{1}\left(t_{p}\right)}{W_{0}}\right)^{2}\right]
$$

For $\mathrm{R}$ is inserted

$$
R\left(t_{p}\right)=\frac{1-\alpha_{p}}{1-\alpha_{p} \alpha_{f}} \text { Pons, eqn.34 }
$$

with

$$
\begin{aligned}
\alpha_{p} & =\exp \left[-\left(e_{n}+c_{n} N_{D}\right) t_{p}\right] \\
\alpha_{f} & =\exp \left[-e_{n}\left(t_{f}-t_{p}\right)\right]
\end{aligned}
$$

Pons eqn's 32 and 33.

for $L_{1}$ is inserted

$$
L_{1} \approx L_{D} \sqrt{2}\left[\ln \left(\beta_{0} t_{p} / \tau_{c}\right)-1\right]^{1 / 2} \quad \text { Pons, eqn.40 }
$$

with $\beta_{0}=1 / \ln \left(2-\alpha_{f}\right)$

$$
\begin{aligned}
L_{0} & =L_{D} \sqrt{2}\left(\frac{E_{F}-E_{T}}{k T}-1\right)^{1 / 2} \\
& =L_{D} \sqrt{2}\left(\ln \left(\frac{\tau_{e}}{\tau_{c}}\right)-1\right)^{1 / 2} \\
W_{0} & =\frac{\epsilon \epsilon_{0} \cdot A_{\text {contact }}}{C_{0}} .
\end{aligned}
$$

98 


\section{A.2.2 Large deep trap concentrations}

In the case of large deep trap concentrations, the amplitude of the refilling kinetics is given by

$$
\frac{\Delta C_{\max }\left(t_{p}\right)}{C_{0}}=\left[1+\frac{2 N_{T}}{N_{D}} F t_{p}\right]^{-1 / 2}-1 \text { Pons, equation } 54
$$

with

$$
F\left(t_{p}\right) \approx \frac{1}{2} \frac{R\left(t_{p}\right)}{W_{0}^{2}}\left\{\left(W_{0}-L_{0}\right)^{2}-\left[W_{1}\left(t_{p}\right)-L_{1}\left(t_{p}\right)\right]^{2}\right\} \quad \text { Pons, equation } 58
$$

with

$$
W_{0}=\frac{\left(N_{T} / N_{D}\right) L_{0}+\left[2 L_{D}^{2}\left(1+N_{T} / N_{D}\right)\left(\left|\frac{q V}{k T}\right|-1\right)-\left(N_{T} / N_{D}\right) L_{0}^{2}\right]^{1 / 2}}{1+N_{T} / N_{D}}
$$

and

$W_{1}=\frac{N_{T}}{N_{D}} \cdot R \cdot L_{1}+\sqrt{\left(\frac{N_{T}}{N_{D}} \cdot R \cdot L_{1}\right)^{2}+2 \cdot\left(1-\frac{N_{T}}{N_{D}}\right) \cdot L_{D}^{2}-\frac{N_{T}}{N_{D}} \cdot R \cdot L_{1}+2 \epsilon \frac{V_{1}}{e N_{d}}}$

(for acceptorlike traps) with

$$
R=\frac{1-\alpha_{p}}{1-\left(N_{T} / N_{D}\right) \alpha_{p}}
$$

with

$$
\alpha_{p}=\exp -c_{n} \cdot\left(N_{D}-N_{T}\right) t_{p}
$$

and

$$
L_{1}=L_{D} \cdot \sqrt{2 \cdot\left(\ln \left(\beta_{0} \frac{t_{p}}{\tau_{c}}\right)-1\right)}
$$




$$
\begin{aligned}
& \beta_{0}=\frac{1}{\ln \left(2-\alpha_{f}\right)} \\
& \alpha_{f}=\exp -e_{n}\left(t_{f}-t_{p}\right) \\
& L_{0}=L_{D} \sqrt{2.0 \ln \left(\frac{1}{\tau_{c}-e_{n}}\right)-1}
\end{aligned}
$$




\section{A.3 Sinks for vacancies and nitrogen}

Once annealed out, the nitrogen-vacancy-related levels stayed absent during subsequent annealing at lower temperatures, which we carried out at some randomly selected samples. This indicates that their annealing reactions are irreversible. Possible loss mechanisms upon the annealing of grown-in nitrogenvacancy complexes are figured out from the analysis of depth profiles of the nitrogen complex X186. The profiles after time-dependent annealing at $680^{\circ} \mathrm{C}$, depicted in fig. A.1, range from the wafer surface to half the thickness of the wafer. They allow a qualitative discussion of the nature of suitable sinks for vacancies and nitrogen, based on the following observations.

After short annealing times, maximum concentrations of the nitrogen annealing complex X186 of $5 \cdot 10^{13} \mathrm{~cm}^{-3}$, in accordance with the maximum concentration described in 3.1.3, are established. With increasing annealing time the concentrations decrease.

All depth profiles show a decrease towards the wafer surface, which appears to operate as a sink over the whole timescale, establishing concentrations in the low $10^{12} \mathrm{~cm}^{-3}$ range.

Additionally, after longer annealing times, the concentration profiles reveal a pronounced loss towards large depths. This indicates an additional sink which is probably not present from the beginning but apparently forms in the course of annealing in the bulk.

The simplest model that conceptually describes the internal sink, assumes an additional agent that diffuses out in the near surface regions or forms a vacancy sink in the bulk regions. In the current temperature regime, in which the sinks operate, a complex formation with oxygen is thinkable. On the basis of binding energies of vacancies to the oxygen dimer around $5 \mathrm{eV}$ as has been derived from $\mathrm{Cz}$-silicon at $1070^{\circ} \mathrm{C}$ [20], the formation of the $\mathrm{O}_{2} \mathrm{~V}$-complex is conceivable from energetic arguments in FZ-silicon, with two or three magnitudes lower oxygen concentration, at temperatures below $800^{\circ} \mathrm{C}$.

Comprehending the whole spectrum of complexes that come into question, requires to consider a great variety of complexes with oxygen, several of which are discussed as precursor states for oxygen nucleation $[136,137,138]$ or as shallow thermal donors, e.g. $[139,140,141,142]$. Certain priority formation reactions are assumed $[142,143]$ to sensitively depend on the initial concentrations of the defects, present in the material, and the thermal prehistory. Therefore, the identification of the prevailing path requires to take into account manifold possibilities. The simulations of the reaction-diffusion equation system for the simulated curves in fig. A.1 uses eight species and qualitatively fits to the experimental data. A quantitative adjustment of the reaction constants is beyond our scope. 


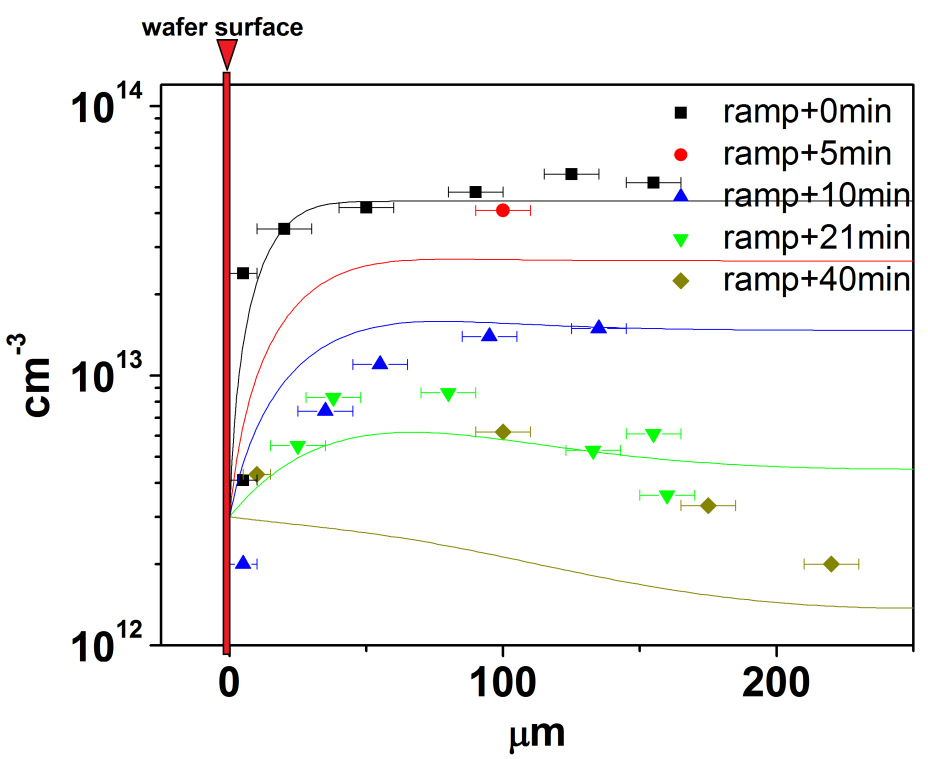

Figure A.1: depth-dependant concentration of the 186K-complex after different annealing periods. 


\section{A.4 DLTS-graphs of nickel marker in-diffusion in crys- talline silicon besides FZ-silicon}

DLTS graphs, as shown in fig. A.2, from nickel marker in-diffusion in standard Czochralski silicon, block cast multicrystalline and quasi monocrystalline silicon revealed no hints for nitrogen vacancy complexes or weakly bound vacancies.

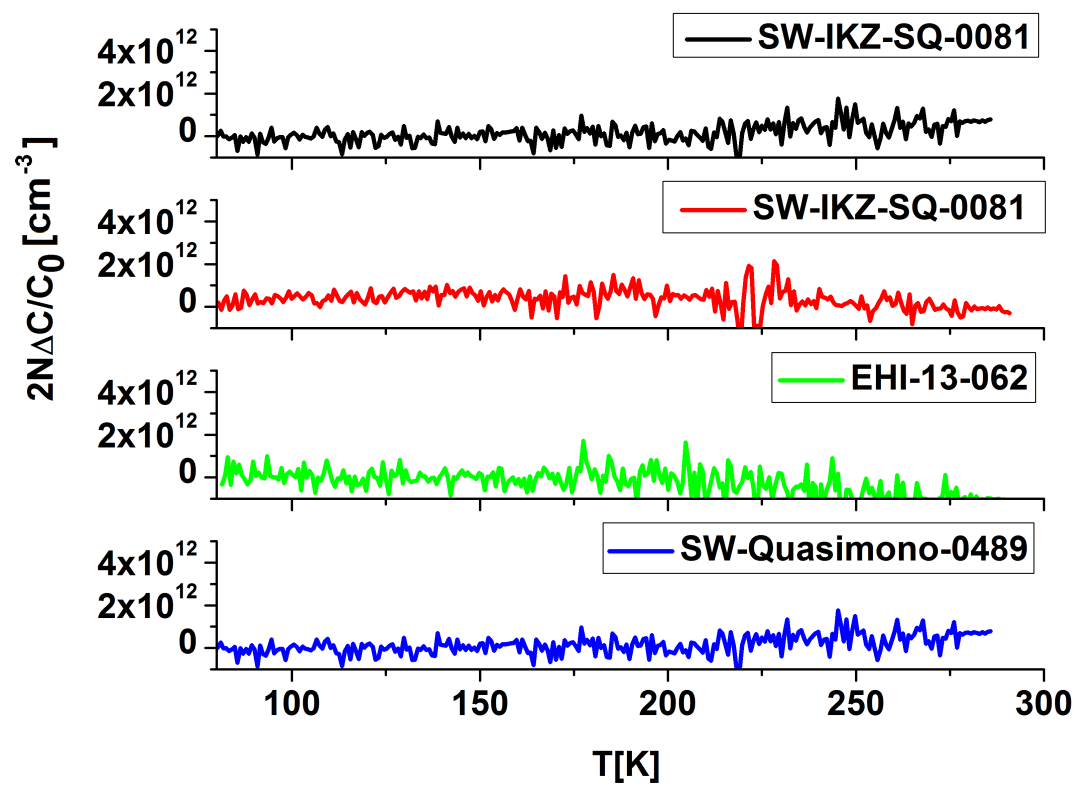

Figure A.2: DLTS analysis of Czochralski silicon, block cast multicrystalline silicon and quasi monocrystalline silicon. No vacancy-nitrogen-complexes or weakly bound vacancies are detected. 


\section{A.5 DLTS graphs from precipitates after different in- diffusion conditions}
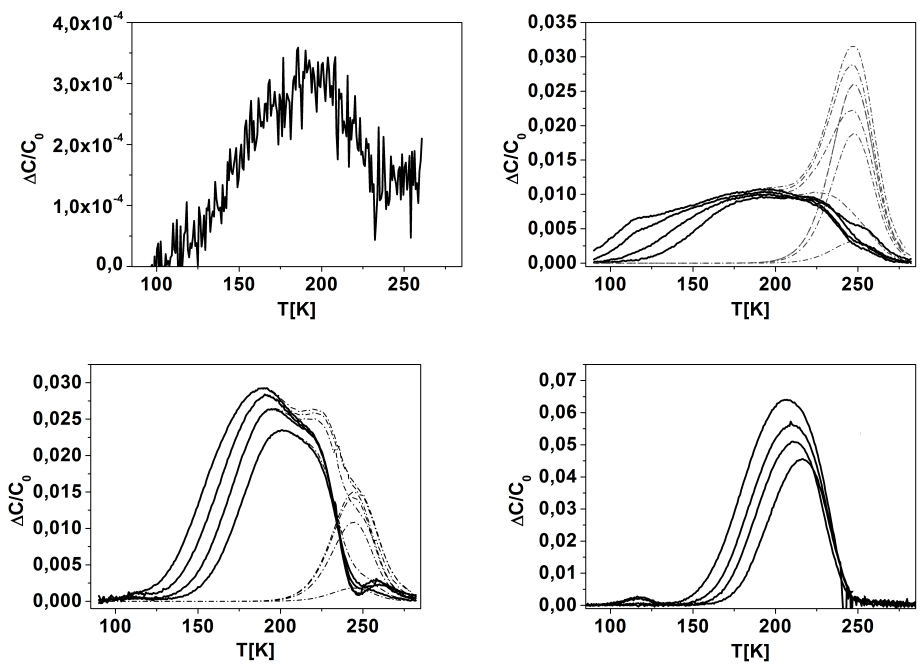

Figure A.3: DLTS signature of $\mathrm{NiSi}_{2}$ precipitates (solid curves), obtained from the subtraction of the substitutional nickel signal (illustrated by the dashed and dotted curves).
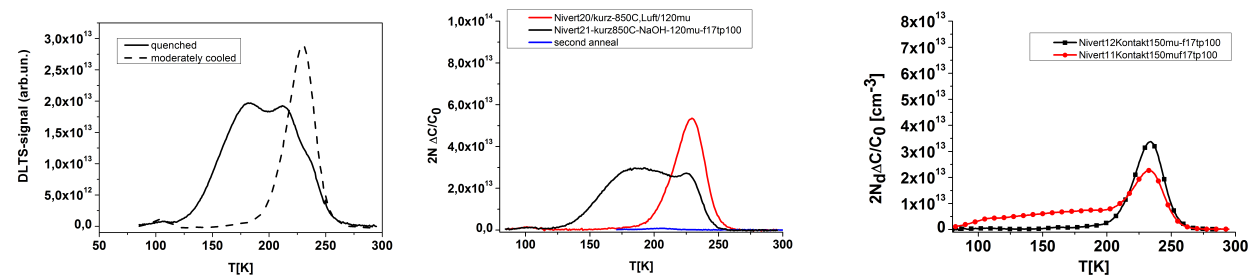

Figure A.4: Comparison of DLTS graphs after nickel in-diffusion finished by quenching with in-diffusion and slow cooling after $900^{\circ} \mathrm{C}, 850^{\circ} \mathrm{C}$ and $750^{\circ} \mathrm{C}$. The spectra show a decrease of the substitutional nickel signal by precipitation. 


\section{A.6 Precipitate density and number of Ni atoms in pre- cipitate}

The density of precipitates $N_{\text {prec }}$ can be determined from the diameter $d$ of the precipitates and the prior concentration of interstitial nickel $C_{N i, i}$ by

$$
N_{\text {prec }}=C_{N i, i} \times\left(h \cdot \pi \cdot \frac{d^{2}}{4} \cdot \rho\right)^{-1} .
$$

The thickness of a precipitate of two lattice planes is $h=0.63 \mathrm{~nm}$. The density of nickel atoms in the precipitate, according to the $\mathrm{NiSi}_{2}$-lattice is $\rho=2.5 \cdot 10^{22} \mathrm{~cm}^{-3}$.

The term in brackets is the number of nickel atoms in the precipitates, according to the assumed number of possible interface states. The density of possible states at the boundary dislocation $N_{R V}([10]$ p. 33) can be calculated by

$$
N_{R V}=\pi \cdot d \cdot n_{R V} \cdot N_{\text {prec }}
$$

The density of dangling bond states per dislocation length can be estimated with the reciprocal burgers vector, $n_{R V}=b^{-1}$, with $b=0.235 \mathrm{~nm}$ ([10], p.33). 


\section{A.7 Energy-dependant EBIC cross section analysis of a $\mathrm{NiSi}_{2}$ precipitate in a ridge structure}

At a ridge structure, illustrated in an overview in fig. A.5, with a ridge thickness of $10 \mu \mathrm{m}$, a systematic variation of the beam energy is performed.
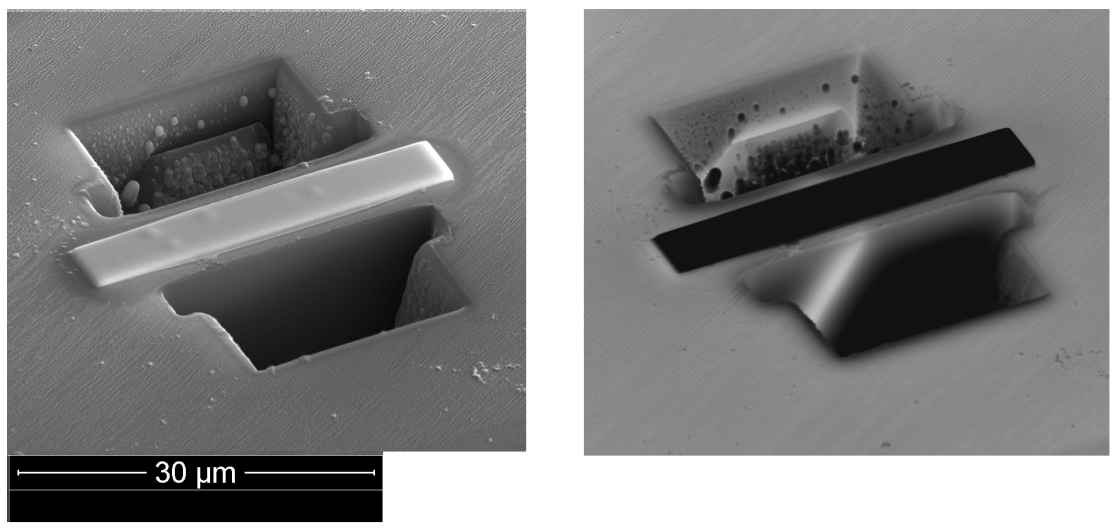

Figure A.5: SEM (left) and EBIC (right) micrographs in tilted and rotated view of a $\mathrm{NiSi}_{2}$-platelet in a ridge.

The examined cross section is shown in fig. A.6 in SEM and EBIC images. The precipitate reveals islands, marked with "i", with an increased thickness which are referred to as thick parts in the following, distinguished from the thin parts.

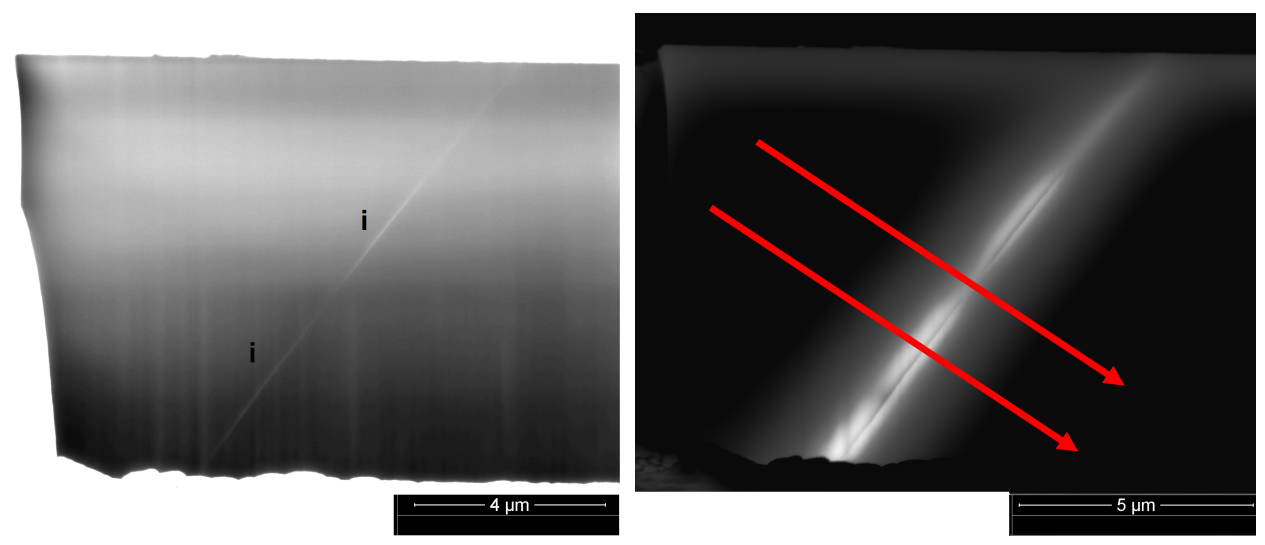

Figure A.6: High magnification SEM (left) and EBIC (right) micrographs of a NiSi $2^{-}$ platelet in a ridge in cross section geometry. To emphasize the dislocation line, a nonlinear image contrast was applied. The red arrows indicate the positions of the following line profiles (see fig. A.7).

The corresponding line profiles across the $\mathrm{NiSi}_{2}$-structure from different acceleration voltages, normalized to their maximum are shown in fig. A.7.

Fig. A.8 shows the maximum detected current. On can see that the current at 

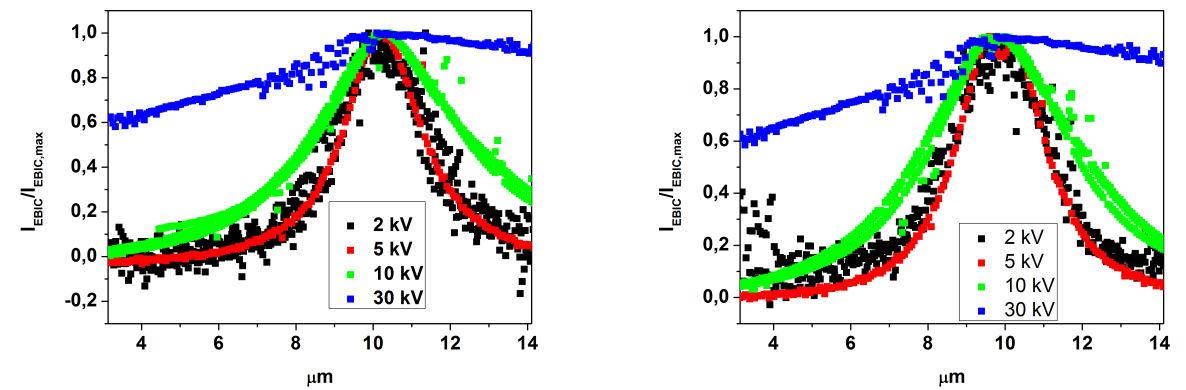

Figure A.7: Normalized line profiles across the structure at the positions from fig. A.6 across a thin part (left) and a thick part (right) with different acceleration voltages.

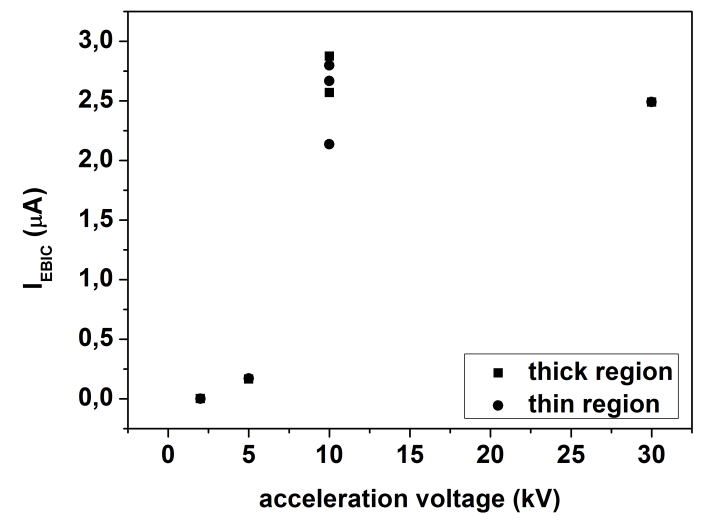

Figure A.8: Maximum detected current of the profiles from fig. A.7.

$2 \mathrm{kV}$ is the lowest, which can be understood when considering that $2 \mathrm{kV}$ has the lowest generation depth giving a hint to high losses by surface recombination at the FIBbed cross section, explained by the dead layer in sec. 3.3.3. It increases with increasing acceleration voltages. 


\section{A.8 experimental parameters}

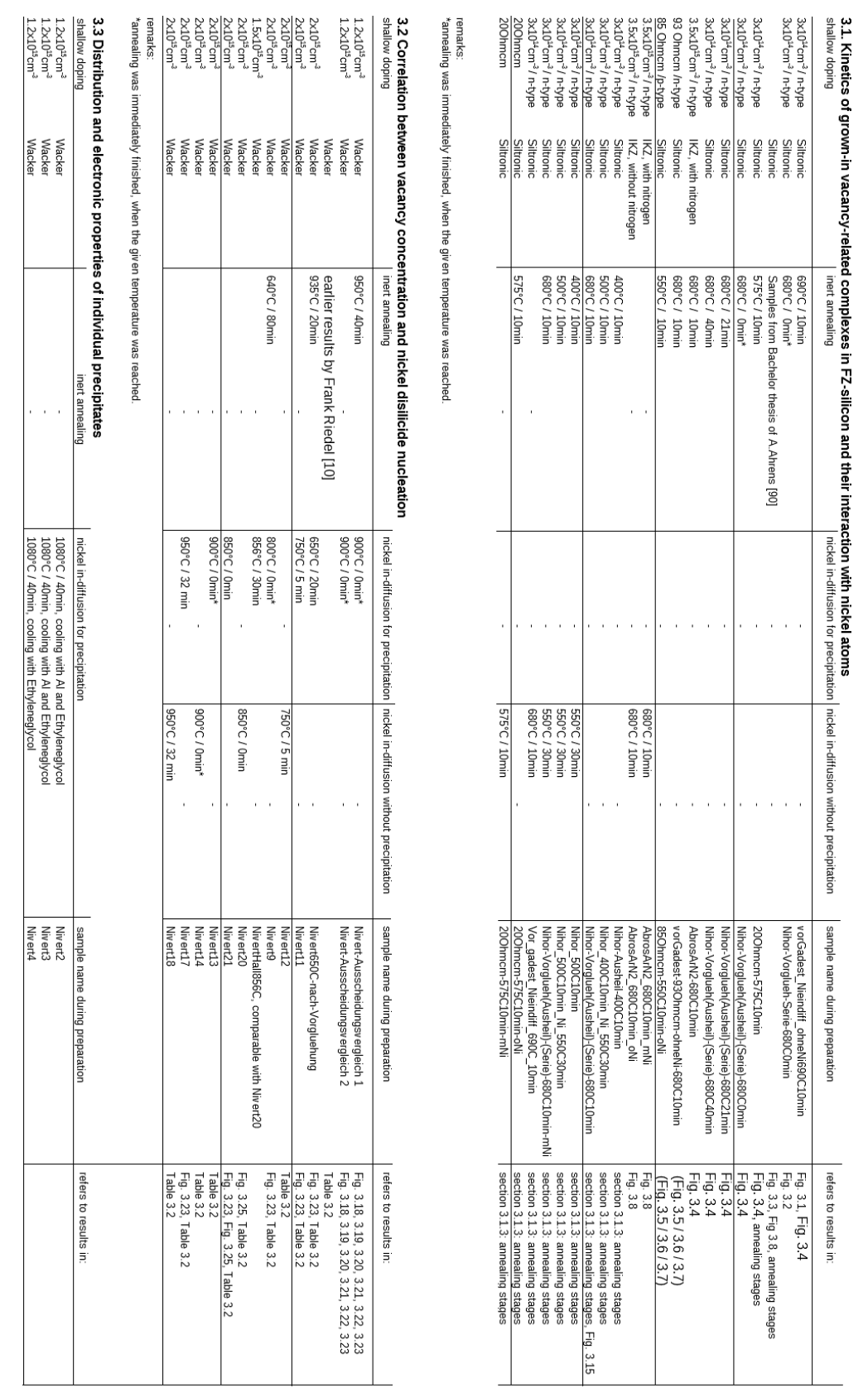




\section{Bibliography}

[1] M. Seibt and V. V. Kveder. "Gettering processes and the role of extended defects". In: Advanced Silicon Materials for Photovoltaic Applications (2012), pp. $127-188$.

[2] T. Buonassisi, A. A. Istratov, M. D. Pickett, M. Heuer, J. P. Kalejs, G. Hahn, M. A. Marcus, B. Lai, Z. Cai, S. M. Heald, T. F. Ciszek, R. F. Clark, D. W. Cunningham, A. M. Gabor, R. Jonczyk, S. Narayanan, E. Sauar, and E. R. Weber. "Chemical natures and distributions of metal impurities in multicrystalline silicon materials". In: Progress in Photovoltaics: Research and Applications 14.6 (2006), pp. 513-531.

[3] E. R. Weber. "Transition metals in silicon". In: Applied Physics A 30.1 (1983), pp. 1-22.

[4] J. Lindroos, D. P. Fenning, D. J. Backlund, E. Verlage, A. Gorgulla, S. K. Estreicher, H. Savin, and T. Buonassisi. "Nickel: A very fast diffuser in silicon". In: Journal of Applied Physics 113.20 (2013), p. 204906.

[5] J. Appenzeller, J Knoch, E Tutuc, M Reuter, and S Guha. "Dual-gate silicon nanowire transistors with nickel silicide contacts". In: Electron Devices Meeting, 2006. IEDM'06. International. IEEE. 2006, pp. 1-4.

[6] M. Yoshida and K. Saito. "Dissociative Diffusion of Nickel in Silicon and Self-Diffusion of Silicon". In: Japanese Journal of Applied Physics 6.5 (1967), pp. $573-581$.

[7] M. K. Bakhadyrkhanov, S. Zainabidinov, and A. Khamidov. "Some characteristics of diffusion and electrotransport of nickel in silicon". In: Sov. Phys. Semicond. 14 (1980), p. 243.

[8] H. Kitagawa, K. Hashimoto, and M. Yoshida. "Diffusion mechanism of nickel and point defects in silicon". In: Japanese Journal of Applied Physics 21.2R (1982), p. 276.

[9] M. Seibt and W. Schröter. "Precipitation behaviour of nickel in silicon". In: Philosophical Magazine A 59(2) (1989), pp. 337-352.

[10] F. Riedel. "Zusammenhang elektrischer und struktureller Eigenschaften von Nickelsilizidausscheidungen in Silizium". Doktorarbeit. Georg-August-Universität, Göttingen, 1994. 
[11] W. Schröter, J. Kronewitz, U. Gnauert, F. Riedel, and M. Seibt. "Bandlike and localized states at extended defects in silicon". In: Physical Review B 52.19 (1995), p. 13726.

[12] R. Tung. "Schottky-Barrier Formation at Single-Crystal Metal-Semiconductor Interfaces". In: Physical Review Letters 52.6 (1984), pp. 461-464.

[13] R. Tung, K. K. Ng, J. M. Gibson, and A. F. J. Levi. "Schottky-barrier heights of single-crystal $\mathrm{NiSi}_{2}$ on $\mathrm{Si}(111)$ : The effect of a surface p-n junction". In: Physical Review B 33 (10 1986), pp. 7077-7090.

[14] A. A. Istratov and E. R. Weber. "Electrical properties and recombination activity of copper, nickel and cobalt in silicon". In: Applied Physics A 66.2 (1998), pp. 123-136.

[15] M. Heuer, T. Buonassisi, A. A. Istratov, M. D. Pickett, M. A. Marcus, A. M. Minor, and E. R. Weber. "Transition metal interaction and Ni-Fe-Cu-Si phases in silicon". In: Journal of Applied Physics 101.12 (2007), p. 123510.

[16] P. Saring, C. Rudolf, L. Stolze, and M. Seibt. "Light-beam-induced current measurements on copper-nickel co-contaminated Cz-silicon bicrystals". In: Materials Science and Engineering: B 159 (2009), pp. 216-218.

[17] M. Seibt, R. Khalil, V. Kveder, and W. Schröter. "Electronic states at dislocations and metal silicide precipitates in crystalline silicon and their role in solar cell materials". In: Applied Physics A 96.1 (2009), pp. 235-253.

[18] M. Shiraishi, J.-U. Sachse, H. Lemke, and J. Weber. "DLTS analysis of nickelhydrogen complex defects in silicon". In: Materials Science and Engineering B 58 (1999), pp. 130-133.

[19] M. A. Khorosheva, V. I. Orlov, N. V. Abrosimov, and V. V. Kveder. "Determination of the Nonequilibrium Concentration of Vacancies in Silicon Crystals by Measuring the Concentration of Nickel Atoms at Lattice Sites". In: Journal of Experimental and Theoretical Physics 110(5) (2010), pp. 769-774.

[20] R. Falster, V. V. Voronkov, and F. Quast. "On the properties of the intrinsic point defects in silicon: a perspective from crystal growth and wafer processing". In: physica status solidi B Basic Research 222.1 (2000), pp. 219-244.

[21] N. V. Abrosimov. Private communication. 2014.

[22] T. Abe, H. Harada, and J.-I. Chikawa. "Microdefects and Impurities in DislocationFree Silicon Crystals". In: MRS Proceedings 14 (Jan. 1982), pp. 1-17.

[23] R. Schmolke, M. Blietz, R. Schauer, D. Zemke, H. Oelkrug, W. von Ammon, U. Lambert, and D. Gräf. "Advanced Silicon Wafers for $0.18 \mu \mathrm{m}$ Design Rule and Beyond: Epi and fLASH!" In: High Purity Silicon VI: Proceedings of the Sixth International Symposium. Vol. 2000. The Electrochemical Society. 2000, p. 3.

[24] V. V. Voronkov and R. Falster. "Out-diffusion of deep donors in nitrogendoped silicon and the diffusivity of vacancies". In: Journal of Applied Physics 112 (2012), p. 013519. 
[25] N. E. Grant, V. P. Markevich, J. Mullins, A. R. Peaker, F. Rougieux, and D. Macdonald. "Thermal activation and deactivation of grown-in defects limiting the lifetime of float-zone silicon". In: physica status solidi (RRL)-Rapid Research Letters 10.6 (2016), pp. 443-447.

[26] M. Jacob, P. Pichler, H. Ryssel, and R. Falster. "Determination of vacancy concentrations in the bulk of silicon wafers by platinum diffusion experiments". In: Journal of Applied Physics 82(1) (1997), pp. 182-191.

[27] J Schön, H Habenicht, M. Schubert, and W Warta. "Understanding the distribution of iron in multicrystalline silicon after emitter formation: Theoretical model and experiments". In: Journal of Applied Physics 109.6 (2011), p. 063717.

[28] N. A. Yarykin and J. Weber. "Identification of copper-copper and copperhydrogen complexes in silicon". In: Semiconductors 47.2 (2013), pp. 275278.

[29] A. Carvalho, D. J. Backlund, and S. K. Estreicher. "Four-copper complexes in $\mathrm{Si}$ and the Cu-photoluminescence defect: A first-principles study". In: Physical Review B 84.15 (2011), p. 155322.

[30] M. E. Alperin, T. C. Hollaway, R. A. Haken, C. D. Gosmeyer, R. V. Karnaugh, and W. D. Parmantie. "Development of the self-aligned titanium silicide process for VLSI applications". In: IEEE Journal of Solid-State Circuits 20.1 (1985), pp. 61-69.

[31] W. M. Weber, L. Geelhaar, A. P. Graham, E. Unger, G. S. Duesberg, M. Liebau, W. Pamler, C. Cheze, H. Riechert, P. Lugli, et al. "Silicon-nanowire transistors with intruded nickel-silicide contacts". In: Nano letters 6.12 (2006), pp. 2660-2666.

[32] B. Lalmi, C. Girardeaux, A. Portavoce, C. Ottaviani, B. Aufray, and J. Bernardini. "Formation and stability of a two-dimensional nickel silicide on Ni (111): An Auger, LEED, STM, and high-resolution photoemission study". In: Physical Review B 85.24 (2012), p. 245306.

[33] Y. Wu, J. Xiang, C. Yang, W. Lu, and C. M. Lieber. "Single-crystal metallic nanowires and metal/semiconductor nanowire heterostructures". In: Nature 430.6995 (2004), pp. 61-65.

[34] K.-C. Lu, W.-W. Wu, H.-W. Wu, C. M. Tanner, J. P. Chang, L. J. Chen, and K. N. Tu. "In situ control of atomic-scale Si layer with huge strain in the nanoheterostructure $\mathrm{NiSi} / \mathrm{Si} / \mathrm{NiSi}$ through point contact reaction". In: Nano Letters 7.8 (2007), pp. 2389-2394.

[35] H Föll. "Lattice Imaging of Silicide-Silicon Interfaces and the Interpretation of Interfacial Defects". In: physica status solidi (a) 69.2 (1982), pp. 779-788.

[36] D Cherns, C. Hetherington, and C. Humphreys. "The atomic structure of the NiSi2-(001) Si interface". In: Philosophical Magazine A 49.1 (1984), pp. 165177. 
[37] C. Hetherington, D Cherns, and C. Humphreys. "The atomic structure of the NiSi 2/(001) Si interface". In: Microscopy of Semiconducting Materials, 1983: Proceedings of the Institute of Physics Conference Held in St. Catherine's College, Oxford, 21-23 March 1983. CRC Press. 1983, p. 89.

[38] G. Ottaviani, K. N. Tu, and J. W. Mayer. "Barrier heights and silicide formation for Ni, Pd, and Pt on silicon". In: Physical Review B 24.6 (1981), p. 3354 .

[39] A. Heinzig, S. Slesazeck, F. Kreupl, T. Mikolajick, and W. M. Weber. "Reconfigurable silicon nanowire transistors". In: Nano Letters 12.1 (2011), pp. 119124.

[40] D. Martin, A. Heinzig, M. Grube, L. Geelhaar, T. Mikolajick, H. Riechert, and W. M. Weber. "Direct probing of Schottky barriers in Si nanowire Schottky barrier field effect transistors". In: Physical Review Letters 107.21 (2011), p. 216807.

[41] C.-Y. Lee, M.-P. Lu, K.-F. Liao, W.-F. Lee, C.-T. Huang, S.-Y. Chen, and L.-J. Chen. "Free-standing single-crystal $\mathrm{NiSi}_{2}$ nanowires with excellent electrical transport and field emission properties". In: The Journal of Physical Chemistry C 113.6 (2009), pp. 2286-2289.

[42] S.-Y. Chen, P.-H. Yeh, W.-W. Wu, U.-S. Chen, Y.-L. Chueh, Y.-C. Yang, S. Gwo, and L.-J. Chen. "Low Resistivity Metal Silicide Nanowires with Extraordinarily High Aspect Ratio for Future Nanoelectronic Devices". In: ACS Nano 5.11 (2011), pp. 9202-9207.

[43] F. Riedel and W. Schröter. "Electrical and structural properties of nanoscale $\mathrm{NiSi}_{2}$ precipitates in silicon". In: Physical Review B 62.11 (2000), p. 7150.

[44] W. Zulehner, G. Harbeke, and M. J. Schulz. "Semiconductor Silicon". In: Springer Series in Material Science. Vol. 13. Springer-Verlag, Berlin, Heidelberg, 1989, pp. 127-152.

[45] W. von Ammon, R. Hölzl, J. Virbulis, E. Dornberger, R. Schmolke, and D. Gräf. "The impact of nitrogen on the defect aggregation in silicon". In: Journal of Crystal Growth 226 (2001), pp. 19-30.

[46] N. Inoue, A. Hashimoto, K. Shingu, and K. Masumoto. "Measurement of Nitrogen Concentration in CZ Silicon Crystals". In: Solid State Phenomena. Vol. 95. Trans Tech Publ. 2004, pp. 489-494.

[47] C Reimann, M Trempa, J Friedrich, and G Müller. "About the formation and avoidance of $\mathrm{C}$ and $\mathrm{N}$ related precipitates during directional solidification of multi-crystalline silicon from contaminated feedstock". In: Journal of Crystal Growth 312.9 (2010), pp. 1510-1516.

[48] V. V. Voronkov and R. Falster. "Grown-in microdefects, residual vacancies and oxygen precipitation bands in Czochralski silicon". In: Journal of Crystal Growth 204.4 (1999), pp. 462-474.

[49] V. V. Voronkov. "THE MECHANISM OF SWIRL DEFECTS FORMATION IN SILICON". In: Journal of Crystal Growth 59 (1982), pp. 625-643. 
[50] G. D. Watkins. "An EPR study of the lattice vacancy in silicon". In: J. Phys. Soc. Japan 18 (1963): Supplement II, pp. 22-27.

[51] G. D. Watkins. Radiation damage in semiconductors. Dunod, Paris, 1965.

[52] G. D. Watkins. "EPR studies of the Lattice Vacancy and Low-Temperature Damage Processes in Silicon". In: Lattice defects in semiconductors, 1974. Invited and contributed papers from the international conference on lattice defects in semiconductors held in Freiburg, 22-25 Jul. 1974. 1975.

[53] Watkins, G. D. and Troxell, J. R. and Chatterjee, A. P. "Vacancies and Interstitials in Silicon". In: Defects and Radiation Effects in Semiconductors, 1978. 1979.

[54] P. Pichler. Intrinsic Point Defects, Impurities, and Their Diffusion in Silicon. Springer-Verlag, Wien, New York, 2004.

[55] V. V. Voronkov and R. Falster. "Intrinsic point defects and impurities in silicon crystal growth". In: Journal of The Electrochemical Society 149.3 (2002), G167-G174.

[56] W. v.Ammon, P. Dreier, W. Hensel, U. Lambert, and L. Koester. "Influence of oxygen and nitrogen on point defect aggregation in silicon single crystals". In: Materials Science and Engineering: B 36 (1996), pp. $33-41$.

[57] N. Inoue and Y. Kawamura. "Infrared defect dynamics - Nitrogen-vacancy complexes in float zone grown silicon introduced by electron irradiation". In: Journal of Applied Physics 123.18 (2018), p. 185701.

[58] D. J. Backlund and S. K. Estreicher. "Ti, Fe, and Ni in Si and their interactions with the vacancy and the $A$ center: A theoretical study". In: Physical Review B 81.23 (2010), p. 235213.

[59] W Schröter and M Seibt. "Solubility and diffusion of transition metal impurities in c-Si". In: EMIS Datareviews Series 20 (1999), pp. 543-560.

[60] H. Zimmermann and H. Ryssel. "Gold and platinum diffusion: The key to the understanding of intrinsic point defect behavior in silicon". In: Applied Physics A 55.2 (1992), pp. 121-134.

[61] V Kveder, M Khorosheva, and M. Seibt. "Interplay of Ni and Au atoms with dislocations and vacancy defects generated by moving dislocations in Si." In: Solid State Phenomena 242 (2016).

[62] J. Weber. Private communication. 2012.

[63] M. Seibt. "Elektronenmikroskopische Untersuchung des Ausscheidungsverhaltens von Nickel in Silizium”. Doktorarbeit. Universität Göttingen, 1986.

[64] J. W. Christian. The Theory of Transformations in Metals and Alloys. I. Pergamon Press, Oxford, 1975.

[65] H. Föll, P. S. Ho, and K. N. Tu. "Transmission electron microscopy of the formation of nickel silicides". In: Philosophical Magazine A 45.1 (1982), pp. 3147 . 
[66] M. Volmer and A Weber. "Keimbildung in übersättigten Gebilden". In: Zeitschrift für physikalische Chemie 119.1 (1926), pp. 277-301.

[67] L Farkas. "Keimbildungsgeschwindigkeit in übersättigten Dämpfen". In: Zeitschrift für physikalische Chemie 125.1 (1927), pp. 236-242.

[68] R Becker and W. Doring. "Kinetic treatment of the nucleation in supersaturated vapors". In: (1954).

[69] U. Gnauert. "Kapazitätstransienten-Spektroskopie an Nickelsilizid-Ausscheidungen in Silizium". Diplomarbeit. Georg-August-Universität Göttingen, 1990.

[70] J. Kronewitz. "Simulation von Kapazitätstransientenspektren ausgedehnter Vielelektronensysteme in Halbleitern". Doktorarbeit. Georg-August-Universität, Göttingen, 1991.

[71] H. Hedemann. "Quantitative Analyse von Kapazitätstransientenspektren ausgedehnter Defekte in Halbleitern". Doktorarbeit. Georg-August-Universität, Göttingen, 1995.

[72] W. Schröter, H. Hedemann, V. Kveder, and F. Riedel. "Measurements of energy spectra of extended defects". In: Journal of Physics: Condensed Matter 14.48 (2002), p. 13047.

[73] M. V. Trushin and O. F. Vyvenko. "Electrical levels of nanoscale $\mathrm{NiSi}_{2}$ precipitates in silicon band gap". In: physica status solidi (c) 4.8 (2007), pp. 30563060 .

[74] J. T. Schick and S. M. Bose. "Electronic structure of a buried $\mathrm{NiSi}_{2}$ or $\mathrm{CoSi}_{2}$ layer in bulk Si". In: Physical Review B 53 (1996), pp. 75273-12612.

[75] M. Kittler, J. Lärz, W. Seifert, M. Seibt, and W. Schröter. "Recombination properties of structurally well defined $\mathrm{NiSi}_{2}$ precipitates in silicon". In: $A p$ plied Physics Letters 58.9 (1991), pp. 911-913.

[76] P. Formanek and M. Kittler. "Direct evidence of internal Schottky barriers at $\mathrm{NiSi}_{2}$ precipitates in silicon by electron holography". In: Journal of Applied Physics 97.6 (2005), p. 063707.

[77] M. Griess. "Zusammenhang struktureller und elektrischer Eigenschaften von Kupfer-Silizid-Ausscheidungen in Silizium”. Doktorarbeit. Georg-August-Universität, Göttingen, 1991.

[78] D. V. Lang. "Deep-level transient spectroscopy: A new method to characterize traps in semiconductors". In: Journal of Applied Physics 45.7 (1974), pp. 3023-3032.

[79] P. Blood and J. W. Orton. The electrical characterization of semiconductors: majority carriers and electron states. Vol. 2. Academic Press, London, Oxford, Boston, New York, San Diego, 1992.

[80] D. Pons. "Accurate determination of the free carrier capture kinetics of deep traps by space-charge methods". In: Journal of Applied Physics 55 (1984), p. 3644 .

[81] O. Voß. "Elektrische Eigenschaften von Gold in plastisch verformtem Silizium". Diplomarbeit. Georg-August-Universität, Göttingen, 2003. 
[82] H. J. Leamy. "Charge collection scanning electron microscopy". In: Journal of Applied Physics 53.6 (1982), R51-R80.

[83] C. Donolato. "An analytical model of SEM and STEM charge collection images of dislocations in thin semiconductor layers: I. Minority carrier generation, diffusion, and collection". In: physica status solidi (a) 65.2 (1981), pp. 649-658.

[84] T. E. Everhart and P. H. Hoff. "Determination of kilovolt electron energy dissipation vs penetration distance in solid materials". In: Journal of Applied Physics 42.13 (1971), pp. 5837-5846.

[85] M. A. Falkenberg, H. Schuhmann, M. Seibt, and V. Radisch. "Localization and preparation of recombination-active extended defects for transmission electron microscopy analysis". In: Review of Scientific Instruments 81.6 (2010), p. 063705.

[86] V. V. Voronkov, G. Voronkova, A. Batunina, R. Falster, L. Moiraghi, and M. Milvidski. "Effects of Annealing on the Electrical Properties of NitrogenDoped Float-Zoned Silicon". In: ECS Transactions 3.4 (2006), pp. 225-235.

[87] T. Abe, H. Harada, N. Ozawa, and K. Adomi. "Deep Level GenerationAnnihilation in Nitrogen Doped FZ Crystals". In: Materials Research Society Symposium Proceedings 59 (1986), p. 537.

[88] K. Murakami, H. Kuribayashi, and K. Masuda. "Electronic Energy Level of Off-Center Substitutional Nitrogen in Silicon". In: Materials Science Forum. Vol. 38. Trans Tech Publications. 1989, pp. 385-390.

[89] W Schröter and M Seibt. "Deep levels of transition metal impurities in c-Si". In: EMIS Datareviews Series 20 (1999), pp. 561-572.

[90] A. Ahrens. "DLTS-Untersuchungen von Leerstellenkonzentrationsprofilen in FZ-Silizium nach Dekoration mit Nickel". Bachelor thesis. Georg-AugustUniversität, Göttingen, 2012.

[91] J. Utzig and W. Schröter. "Donor and acceptor behaviour of gold in silicon". In: Applied Physics Letters 45.7 (1984), pp. 761-763.

[92] T. Abe. "Deep Level Generation-Annihilation in Nitrogen Doped FZ Crystals". In: Proceedings of the Forum on the Science and Technology of Silicon Materials. Ed. by H. Yamada-Kaneta and K. Sumino. 2001, pp. 19-31.

[93] J. Stephen, B. J. Smith, G. W. Hinder, D. C. Marshall, and E. M. Wittam. "Some Observations on High Energy Nitrogen Implantations in Silicon". In: Ion Implantation in Semiconductors. Springer, 1971, pp. 489-494.

[94] J. B. Mitchell, P. P. Pronko, J. Shewchun, D. A. Thompson, and J. A. Davies. "Nitrogen- implanted silicon. I. Damage annealing and lattice location". In: Journal of Applied Physics 46.1 (1975), pp. 332-334.

[95] J. B. Mitchell, J. Shewchun, D. A. Thompson, and J. A. Davies. "Nitrogenimplanted silicon. II. Electrical properties". In: Journal of Applied Physics 46.1 (1975), pp. 335-343. 
[96] F. D. M. Haldane and P. W. Anderson. "Simple model of multiple charge states of transition-metal impurities in semiconductors". In: Physical Review B 13.6 (1976), p. 2553.

[97] A. Oshiyama, M. Saito, and O. Sugino. "Covalency, elasticity and electron correlation in Si vacancies". In: Applied Surface Science 85 (1995), pp. 239245 .

[98] D. J. Backlund and S. K. Estreicher. "Structural, electrical, and vibrational properties of Ti-H and Ni-H complexes in Si". In: Physical Review B 82.15 (2010), p. 155208.

[99] H. Sawada and K. Kawakami. "First-principles calculation of the interaction between nitrogen atoms and vacancies in silicon". In: Physical Review B 62 (3 2000), pp. 1851-1858.

[100] F. Quast, P. Pichler, H. Ryssel, and R. Falster. "Vacancy-Nitrogen Complexes in Float-Zone Silicon". In: Proceedings of SPIE - The International Society for Optical Engineering. 2000, pp. 156-163.

[101] M. Jacob, P. Pichler, H. Ryssel, and R. Falster. "Determination of vacancy concentrations in the bulk of silicon wafers by platinum diffusion experiments". In: Journal of Applied Physics 82.1 (1997), pp. 182-191.

[102] V. V. Kveder, M. A. Khorosheva, and M. Seibt. "Interplay of Ni and Au Atoms with Dislocations and Vacancy Defects Generated by Moving Dislocations in Si". In: Solid State Phenomena 242 (2015), p. 147.

[103] M. A. Khorosheva, V. V. Kveder, and M. Seibt. "On the nature of defects produced by motion of dislocations in silicon". In: physica status solidi (a) 212.8 (2015), pp. 1695-1703.

[104] M. Seibt, A. Sattler, C. Rudolf, O. Voß, V. V. Kveder, and W. Schröter. "Gettering in silicon photovoltaics: current state and future perspectives". In: physica status solidi (a) 203.4 (2006), pp. 696-713.

[105] M. A. Falkenberg. "Metal Impurity Redistribution in Crystalline Silicon for Photovoltaic Application". Doktorarbeit. Georg-August-Universität, Göttingen, 2014.

[106] D. Cherns, G. R. Anstis, J. L. Hutchinson, and S. J. C. H. "Atomic structure of the $\mathrm{NiSi}_{2} /(111)$ Si interface". In: Philiosophical Magazine A 46 (1982), p. 849.

[107] F. S. d'Aragona. "Dislocation etch for (100) planes in silicon". In: Journal of The Electrochemical Society 119.7 (1972), pp. 948-951.

[108] G. K. Metal impurities in silicon-device fabrication. Springer Science \& Business Media, Luxemburg, Berlin, Heidelberg, 2000.

[109] P. S. Plekhanov and T. Y. Tan. "Schottky effect model of electrical activity of metallic precipitates in silicon". In: Applied Physics Letters 76.25 (2000), pp. 3777-3779.

[110] M Seibt and K Graff. "Characterization of haze-forming precipitates in silicon". In: Journal of Applied Physics 63.9 (1988), pp. 4444-4450. 
[111] P. Haasen. Physical Metallurgy. Cambridge University Press, Cambridge, 1978.

[112] H Lemke. "Substitutional transition metal defects in silicon grown-in by the float zone technique". In: Proceedings of the Fourth International Symposium on High Purity Silicon. Vol. 96. The Electrochemical Society. 1996, p. 272.

[113] T. Sinno, R. A. Brown, W. von Ammon, and E. Dornberger. "On the Dynamics of the Oxidation-Induced Stacking-Fault Ring in as-grown Czochralski silicon crystals". In: Applied Physics Letters 70.17 (1997), pp. 2250-2252.

[114] W. Schröter and H. Cerva. "Interaction of point defects with dislocations in silicon and germanium: electrical and optical effects". In: Solid State Phenomena. Vol. 85. Trans Tech Publ. 2002, pp. 67-144.

[115] B. Hammer and J. Nørskov. "Electronic factors determining the reactivity of metal surfaces". In: Surface Science 343.3 (1995), pp. 211-220.

[116] G. D. Watkins. "Intrinsic Point Defects in Semiconductors 1999". In: Handbook of Semiconductor Technology: Electronic Structure and Properties of Semiconductors (2000), pp. 121-165.

[117] J Utzig. "Properties of cobalt in FZ and CZ silicon studied by Mössbauer spectroscopy". In: Journal of applied physics 64.7 (1988), pp. 3629-3633.

[118] R. J. Falster, V. V. Voronkov, V. Resnick, and M. Milvidski. "Morphological Transformation of Oxide Particles and Thresholds for Effective Gettering in Silicon". In: Gettering and Defect Engineering in Semiconductor Technology XI. Vol. 108. Solid State Phenomena. Trans Tech Publications, Oct. 2005, pp. $97-102$.

[119] Y. Ohno, K. Inoue, K. Kutsukake, M. Deura, T. Ohsawa, I. Yonenaga, H. Yoshida, S. Takeda, R. Taniguchi, H. Otubo, et al. "Nanoscopic mechanism of $\mathrm{Cu}$ precipitation at small-angle tilt boundaries in Si". In: Physical Review B 91.23 (2015), p. 235315.

[120] S. Hudelson, B. K. Newman, S. Bernardis, D. P. Fenning, M. I. Bertoni, M. A. Marcus, S. C. Fakra, B. Lai, and T. Buonassisi. "Retrograde melting and internal liquid gettering in silicon". In: Advanced Materials 22.35 (2010), pp. 3948-3953.

[121] W. Schröter, M. Seibt, and D. Gilles. "High-Temperature Properties of 3d Transition Elements in Silicon". In: Electronic Structure and Properties of Semiconductors. Ed. by R. W. Cahn, P. Haasen, and E. J. Kramer. Vol. 4. Materials Science and Technology. VCH Verlagsgesellschaft, Weinheim, New York, Basel, Cambridge, 1991.

[122] P. Krenckel, P. Saring, M. A. Falkenberg, V. Kveder, and M. Seibt. "Interaction of iron with extended defects in multicrystalline silicon studied by local gettering". In: Energy Procedia 38 (2013), pp. 582-588.

[123] P. M. Haney, H. P. Yoon, B. Gaury, and N. B. Zhitenev. "Depletion region surface effects in electron beam induced current measurements". In: Journal of Applied Physics 120.9 (2016), p. 095702. 
[124] P. Peretzki, B. Ifland, C. Jooss, and M. Seibt. "Low energy scanning transmission electron beam induced current for nanoscale characterization of $\mathrm{p}^{-}$ n junctions". In: physica status solidi (RRL)-Rapid Research Letters 11.1 (2017), p. 1600358.

[125] P Peretzki and M. Seibt. Private communication. 2018.

[126] R. T. Tung. "The physics and chemistry of the Schottky barrier height". In: Applied Physics Reviews 1.1 (2014), p. 011304.

[127] J. D. Jackson. Classical electrodynamics. John Wiley \& Sons, 2012.

[128] L. D. Landau and E. Lifshitz. Lehrbuch der theoretischen Physik. Vol. 8: Elektrodynamikdr Kontinua, 5. berichtigte Auflage. Akademie-Verlag, Berlin, 1985.

[129] M. Kittler, X. Yu, T. Mchedlidze, T. Arguirov, O. F. Vyvenko, W. Seifert, M. Reiche, T. Wilhelm, M. Seibt, O. Voß, A. Wolf, and W. Fritzsche. "Regular Dislocation Networks in Silicon as a Tool for Nanostructure Devices Used in Optics, Biology, and Electronics". In: Small 3.6 (2007), pp. 964-973.

[130] W. Yi, J. Chen, S. Ito, K. Nakazato, T. Kimura, T. Sekiguchi, and K. Fujiwara. "Investigation of Si Dendrites by Electron-Beam-Induced Current". In: ().

[131] C.-L. Hsin and S.-S. Deng. "Epitaxial nickel disilicide with low resistivity and excellent reliability". In: Nanotechnology 27.6 (2016), p. 065704.

[132] F. C. Frank and D. Turnbull. "Mechanism of diffusion of copper in germanium". In: Physical Review 104.3 (1956), p. 617.

[133] U. Gösele, W. Frank, and A. Seeger. "Mechanism and kinetics of the diffusion of gold in silicon". In: Applied Physics A: Materials Science \& Processing 23.4 (1980), pp. 361-368.

[134] H. Kitagawa and M. Yoshida. "On the distinction between the dissociative and kick-out mechanisms for site exchange in silicon". In: Japanese Journal of Applied Physics 31.9A (1992), p. 2859.

[135] T. Sinno, R. A. Brown, W. von Ammon, and E. Dornberger. "Point Defect Dynamics and the Oxidation-Induced Stacking-Fault Ring in CzochralskiGrown Silicon Crystals". In: Journal of the Electrochemical Society 145.1 (1998), pp. 302-318.

[136] A. Karoui, F. S. Karoui, G. A. Rozgonyi, and D. Yang. "Oxygen precipitation in nitrogen doped Czochralski silicon wafers. I. Formation mechanisms of near-surface and bulk defects". In: Journal of Applied Physics 96.6 (2004), pp. 3255-3263.

[137] A. Karoui and G. A. Rozgonyi. "Oxygen precipitation in nitrogen doped Czochralski silicon wafers. II. Effects of nitrogen and oxygen coupling". In: Journal of Applied Physics 96.6 (2004), pp. 3264-3271. 
[138] F. S. Karoui and A. Karoui. "A density functional theory study of the atomic structure, formation energy, and vibrational properties of nitrogen-vacancyoxygen defects in silicon". In: Journal of Applied Physics 108.3 (2010), pp. 033513033513.

[139] M. Suezawa, K. Sumino, H. Harada, and T. Abe. "The nature of nitrogenoxygen complexes in silicon". In: Japanese Journal of Applied Physics 27.1 part 1 (1988), pp. 62-67.

[140] R. Jones. "Ab initio calculations on thermal donors in Si: an over-coordinated O atom model for the NL10 and NL8 centres". In: Semiconductor Science and Technology 5.3 (1990), p. 255.

[141] C. P. Ewels, R. Jones, S. Öberg, J. Miro, and P. Deak. "Shallow thermal donor defects in silicon". In: Physical Review Letters 77.5 (1996), p. 865.

[142] H. C. Alt and H. E. Wagner. "Thermal stability and vibrational spectroscopy of $\mathrm{N}-\mathrm{O}$ shallow donor centers in silicon". In: Journal of Applied Physics 106.10 (2009), p. 103511.

[143] N. Fujita, R. Jones, S. Öberg, and P. R. Briddon. "Nitrogen related shallow thermal donors in silicon". In: Applied Physics Letters 91.5 (2007), pp. 051914 051914 . 


\section{Danksagung}

Mein erster Dank gilt Professor Michael Seibt, der mich während meiner langen Zeit in seiner Arbeitsgruppe auf besondere Weise geprägt hat. So habe ich erlebt, wie sein Interesse über das Fachliche hinausgehend dem persönlichen Wohl seiner Studenten gilt. Während der Krankheit meines Vaters habe ich - selbst in kurzen Gesprächen - Michaels menschliche Unterstützung erfahren, die mir sehr geholfen hat und letztendlich auch die Vollendung der Arbeit ermöglichte. Weitere besondere Eigenschaften sind sein Scharfsinn, sein Witz und seine Gelassenheit, die mich tief beeindrucken.

Grossen Dank möchte ich auch allen Kollegen in der Arbeitsgruppe aussprechen, insbesondere meinem langjährigen aktuellen Büro-Nachbarn Patrick Peretzki und meinen früheren Büro-Nachbarn Dr. Carsten Rudolf und Dr. Oliver Voss, die in hilfreichen Diskussionen und Hinweisen zum Gelingen der Arbeit beigetragen haben, sowie bei Prof. Vitaly Kveder, der auf besondere Weise Begeisterung vermitteln kann und mich mit wertvollen Diskussionen und Tips beim Experimentieren für die Arbeit unterstützte. Außerdem bedanke ich mich bei Bettina Schlieper-Ludewig, die mich bei den Laborarbeiten mit Rat und Tat unterstützt hat.

Bei Arne Ahrens bedanke ich mich vor allem für die gute Zusammenarbeit während seiner Bachelorarbeit, aus der viele Erkenntnisse für diese Arbeit entstanden sind, sowie für seinen unerbittlichen Eifer beim kritischen Korrekturlesen dieser Arbeit und die Unterstützung bei der Einarbeitung, die mir in der Endspurtphase sehr geholfen hat.

Bei Felix Hippmann und Andreas Juhnke bedanke ich mich für die Unterstützung bei der Formatierung und für ihr kritisches Korrekturlesen.

Besonders bedanke ich mich bei meinen Eltern Brunhild und Ehrhard (gest. 9. Dezember 2016) Saring sowie bei meiner Frau Maike und meinen Schwiegereltern Ester und Bernd für ihre Unterstützung und Geduld. In die Zeit der Doktorarbeit fallen auch die Geburten meiner beiden Töchter Miriam Louisa (*19. Juni.2014) und Johanna Marie (*6. März 2017) die sich auch über die fertige Arbeit freuen. Sie haben mir schon früh gezeigt, dass ein Lächeln manchmal mehr sagt als tausend Worte. 